\title{
Neurobiological Mechanisms of Nicotine Reward and Aversion
}

Lauren Wills, Jessica L. Ables, Kevin M. Braunscheidel, Stephanie P.B. Caligiuri, Karim S. Elayouby, Clementine Fillinger, Masago Ishikawa, Janna K. Moen, and Paul J. Kenny

Nash Family Department of Neuroscience, Icahn School of Medicine at Mount Sinai, One Gustave L. Levy Place, New York, New York

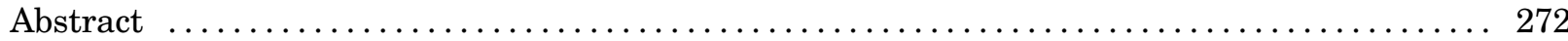

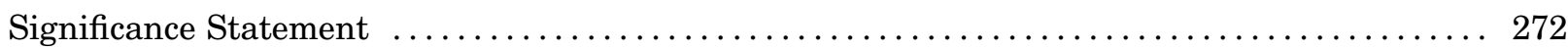

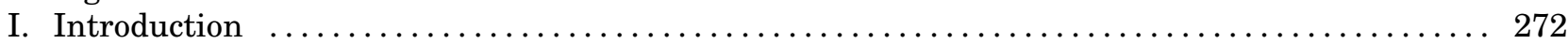

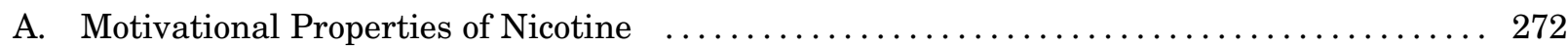

B. Structural Architecture of $\mathrm{nAChRs}$. . . . . . . . . . . . . . . . . . . . . . . . . . . 273

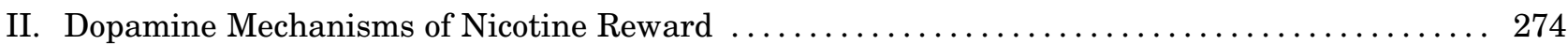

A. nAChRs in Brain Dopamine Systems . . . . . . . . . . . . . . . . . . . . . . . . . . . . . 274

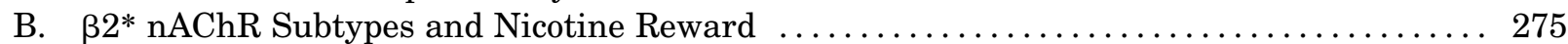

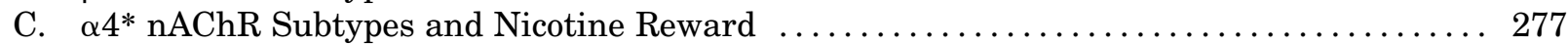

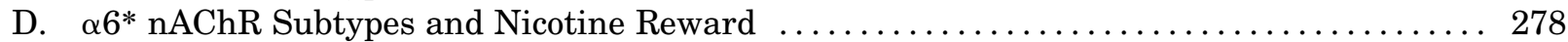

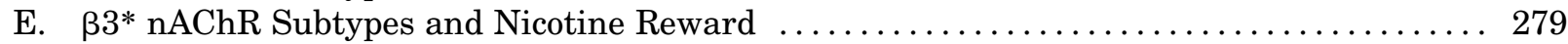

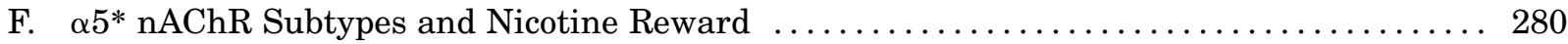

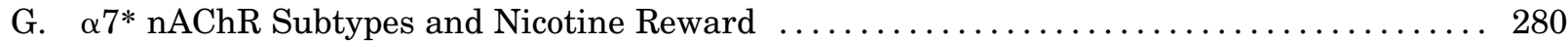

H. Nicotine Modifies Impulse-Dependent and -Independent Accumbal Dopamine Release . . 281

III. Dopamine Mechanisms of Nicotine Aversion . . . . . . . . . . . . . . . . . . . . . . . . . . 282

IV. Cellular Mechanisms in VTA of Nicotine Reward and Aversion $\ldots \ldots \ldots \ldots \ldots \ldots \ldots \ldots \ldots 283$

A. Balance between nAChR Signaling in VTA Dopaminergic and GABAergic Neurons . . ... 283

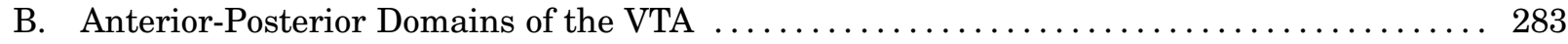

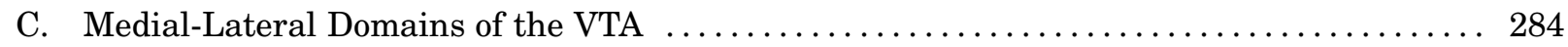

V. Nondopamine Mechanisms of Nicotine Aversion $\ldots \ldots \ldots \ldots \ldots \ldots \ldots \ldots \ldots \ldots \ldots \ldots \ldots 285$

A. Human Genetics Reveal nAChR Subtypes that Regulate Nicotine Intake . . . . . . . 285

B. $\alpha 5^{*}, \alpha 3^{*}$, and $\beta 4^{*}$ nAChRs Regulate Nicotine Avoidance $\ldots \ldots \ldots \ldots \ldots \ldots \ldots \ldots \ldots 286$

C. Low-Affinity nAChRs Are Enriched in Medial Habenula and Interpeduncular Nucleus .. 287

D. nAChR Signaling in the mHb-IPn Circuit Regulates Nicotine Avoidance ............ 287

E. nAChR Signaling in mHb-IPn Circuit Regulates the Reward-Inhibiting Effects of

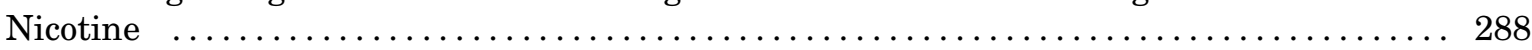

F. Stoichiometries of nAChRs Expressed in the

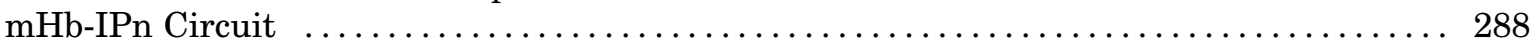

G. nAChRs in IPn Regulate Nicotine Reward and Aversion . . . . . . . . . . . . . . . . . 290

H. Efferent Projections from IPn Regulate Nicotine Reward and Aversion . . . . . . . . . . . 291

I. Afferent Projections to IPn Regulate Nicotine Reward and Aversion . . . . . . . . . . ... 293

J. Other Reward and Aversion Signaling Mechanisms in the mHb-IPn Circuit ......... 293

K. Nicotine Actions in the Periphery Impact Reward and Aversion Behaviors . . ........ 295

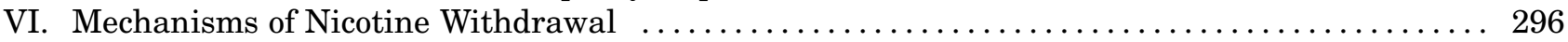

A. Nicotine Withdrawal Syndrome in Humans and Rodents . . . . . . . . . . . . . . . . 296

B. $\quad \beta 2 *$ nAChRs Regulate Affective but Not Physical Components of Nicotine Withdrawal ... 296

C. Other nAChR Subtypes Contribute to Affective Components of Nicotine Withdrawal ... 297

D. Dopamine Transmission Contributes to Affective Components of Nicotine Withdrawal ... 297

E. $\beta 4^{*}$ nAChRs Regulate Physical but Not Affective Components of Nicotine Withdrawal ... 298

Address correspondence to: Dr. Paul Kenny, Nash Family Department of Neuroscience, Icahn School of Medicine at Mount Sinai, 1470 Madison Ave., New York, NY 10029. E-mail: paul.kenny@mssm.edu

This work was supported by National Institutes of Health National Institute on Drug Abuse [Grants DA020686 and DA045649] (to P.J.K.).

No author has an actual or perceived conflict of interest with the contents of this article.

dx.doi.org/10.1124/pharmrev.121.000299. 
F. nAChR Signaling in mHb-IPn Circuit Regulates Nicotine Withdrawal . . ......... 299

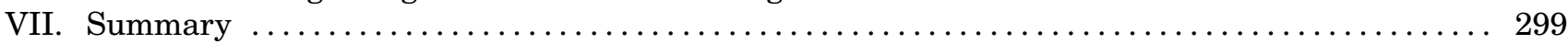

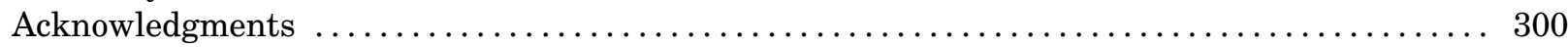

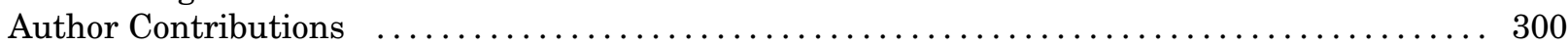

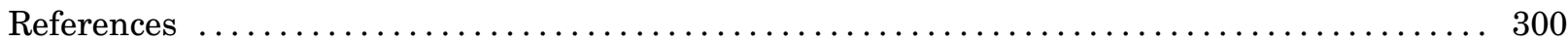

\begin{abstract}
Neuronal nicotinic acetylcholine receptors (nAChRs) regulate the rewarding actions of nicotine contained in tobacco that establish and maintain the smoking habit. nAChRs also regulate the aversive properties of nicotine, sensitivity to which decreases tobacco use and protects against tobacco use disorder. These opposing behavioral actions of nicotine reflect nAChR expression in brain reward and aversion circuits. nAChRs containing $\alpha 4$ and $\beta 2$ subunits are responsible for the high-affinity nicotine binding sites in the brain and are densely expressed by reward-relevant neurons, most notably dopaminergic, GABAergic, and glutamatergic neurons in the ventral tegmental area. High-affinity nAChRs can incorporate additional subunits, including $\beta 3, \alpha 6$, or $\alpha 5$ subunits, with the resulting nAChR subtypes playing discrete and dissociable roles in the stimulatory actions of nicotine on brain dopamine transmission. nAChRs in brain dopamine circuits also participate in aversive reactions to nicotine and the negative affective state experienced during nicotine withdrawal. nAChRs containing $\alpha 3$
\end{abstract}

and $\beta 4$ subunits are responsible for the low-affinity nicotine binding sites in the brain and are enriched in brain sites involved in aversion, including the medial habenula, interpeduncular nucleus, and nucleus of the solitary tract, brain sites in which $\alpha 5 \mathrm{nAChR}$ subunits are also expressed. These aversion-related brain sites regulate nicotine avoidance behaviors, and genetic variation that modifies the function of nAChRs in these sites increases vulnerability to tobacco dependence and smoking-related diseases. Here, we review the molecular, cellular, and circuit-level mechanisms through which nicotine elicits reward and aversion and the adaptations in these processes that drive the development of nicotine dependence.

Significance Statement_- Tobacco use disorder in the form of habitual cigarette smoking or regular use of other tobacco-related products is a major cause of death and disease worldwide. This article reviews the actions of nicotine in the brain that contribute to tobacco use disorder.

\section{Introduction}

\section{A. Motivational Properties of Nicotine}

Tobacco smoking results in more than 5 million deaths each year worldwide (WHO, 2008), and it is predicted that approximately 0.6 billion current smokers will die from smoking-related illnesses (Ezzati and Lopez, 2003; Doll et al., 2004; Coe et al., 2005; Mathers and Loncar, 2006). Even in nonsmokers tobacco can be deadly, with over 880,000 people worldwide estimated to die annually year from diseases related to secondhand smoke exposure (Oberg et al., 2011). According to the Centers for Disease Control and Prevention, an estimated $14 \%$ of adults in the United States ( $~ 34.1$ million people) were current cigarette smokers in 2019 (Statistics, 2018). This level of tobacco use represents an all-time low and the culmination of a trend that has been apparent since the mid1960s, when approximately $45 \%$ of adults in the United States were current smokers. In contrast to this progress in reducing cigarette smoking in adults, use of noncigarette tobacco products, most notably electronic delivery devices, has increased dramatically over the past 10 years, particularly in school-age children. From 2011 to 2019, use of electronic cigarettes (e-cigarettes) among children increased $>1500 \%$, with almost 3 million children initiating e-cigarette use (7900 per day) in 2019 alone. Strikingly, $\sim 30 \%$ of high school students report use of a tobacco product. These young e-cigarette users show increased propensity to use conventional cigarettes and other combustible tobacco products relative to children who do not use e-cigarettes (Leventhal et al., 2015). As might be expected, habitual tobacco use is associated with diseases of the airways, including lung, larynx, and mouth cancers; chronic obstructive pulmonary disease; and asthma (Collaborators, 2017). Cigarette smoking is responsible for $\sim 90 \%$ of all lung cancers in the United States (Mokdad et al., 2004), with more people dying from this smoking-related disease than any other type of cancer. Tobacco smoking and e-cigarette use are also a

\footnotetext{
ABBREVIATIONS: aVTA, anterior VTA; $\mathrm{CB}_{1}$, cannabinoid 1; CNS, central nervous system; CPA, conditioned place avoidance; CPP, conditioned place preference; $\mathrm{CRF}$, corticotropin-releasing hormone; $\mathrm{DH} \beta \mathrm{E}$, dihydro- $\beta$-erythroidine; Ex- 4 , exendin- 4 ; GluN1, glutamate ionotropic receptor NMDA subunit 1; GluN3A, glutamate ionotropic receptor NMDA subunit 3A; GLP-1, glucagon-like peptide 1; GPCR, G protein-coupled receptor; HCN, hyperpolarization-activated cyclic nucleotide-gated channel; ICSS, intracranial self-stimulation; IPn, interpeduncular nucleus; LDTg, laterodorsal tegmental nucleus; LHb, lateral habenula; 18-MC, 18-methoxycoronaridine; mHb, medial habenula; MLA, methyllycaconitine; mPFC, medial prefrontal cortex; NAc, nucleus accumbens; nAChR, nicotinic acetylcholine receptor; NMDA, $N$-methyl-D-aspartic acid; nTS, nucleus tractus solitarius/nucleus of the solitary tract; PDE2A, phosphodiesterase 2A; PPTg, pedunculopontine tegmental nucleus; pVTA, posterior VTA; RMTg, rostromedial tegmental nucleus; shRNA, short interfering ribonucleic acid; TCF7L2, transcription factor 7-like 2; VH, ventral hippocampus; VTA, ventral tegmental area; VTA ${ }^{\text {MED }}$, medial VTA; VTA ${ }^{\text {LAT }}$, lateral VTA.
} 
major cause of nonrespiratory system diseases, including type 2 diabetes and cardiovascular disease (Rostron et al., 2014; Collaborators, 2017; Hedman et al., 2018; Xie et al., 2020). Smokers who quit before the onset of tobacco-related illness can largely avoid the increased mortality risk (Doll et al., 1994; Peto et al., 2000). Despite awareness of the dangers of smoking, approximately $80 \%$ of current smokers attempting to quit will relapse within the first month of abstinence (Benowitz, 2009). Current smoking cessation medications have limited utility. In smokers attempting to quit, $\sim 23 \%$ treated with Chantix (varenicline) and $\sim 16 \%$ treated with Zyban (bupropion) remain abstinent after 1 year compared with $\sim 9 \%$ of those treated with placebo (Knight et al., 2009). Pharmacotherapy is therefore an effective strategy to aid smoking cessation, but there remains considerable risk of relapse even when using the most effective medications currently available. The development of more efficacious smoking-cessation therapeutics would prevent the premature death of hundreds of thousands of people each year and is perhaps the most cost-saving intervention possible within a modern healthcare system (Knight et al., 2009). Leveraging our growing understanding of the basic neurobiological mechanisms of tobacco use disorder will likely lead to more effective smoking-cessation therapeutics.

Nicotine is the major rewarding component responsible for the reinforcing properties of cigarette smoke, which drive the development of tobacco use disorder (Stolerman and Jarvis, 1995). Nicotine intake produces a subjectively pleasant experience (reward), the obtaining of which increases the likelihood that smoking behavior will occur again (reinforcement) (Fowler and Kenny, 2011). Nicotine has both positive and negative reinforcing properties, meaning that the drug enhances the activity of brain reward circuits (positive reinforcement) while attenuating the activity of brain aversion circuits during withdrawal (negative reinforcement) (Kenny and Markou, 2001). Consistent with a key role in tobacco use disorder, nicotine is volitionally self-administered via intravenous infusions by humans (Harvey et al., 2004), nonhuman primates (Goldberg and Spealman, 1982), dogs (Risner and Goldberg, 1983), rats (Corrigall and Coen, 1989; DeNoble and Mele, 2006), and mice (Fowler and Kenny, 2011). Nicotine self-administration under fixed-ratio schedules of reinforcement produces an inverted U-shaped doseresponse curve similar to other reinforcing drugs, such as opioids and cocaine. The shape of the nicotine doseresponse curve reflects competing positive and negative effects of nicotine at different doses. Increased responding for nicotine over the ascending portion of the doseresponse curve reflects the intensifying rewarding effects of nicotine as the amount of drug per infusion increases. Decreased responding over the descending portion of the curve likely reflects increasing aversive properties of nicotine that motivate avoidance behaviors.
Consistent with the notion that nicotine elicits both reinforcing and punishing effects is the observation that nonhuman primates volitionally self-administer the same doses of nicotine that they will work to avoid when they are delivered nonvolitionally (Goldberg and Spealman 1982, 1983; Spealman and Goldberg, 1982). Factors other than aversion also contribute to the descending arm of the dose-response curve seen in animals responding under fixed-ratio schedules. For example, more rapid "satiation" when higher doses are available, which means that lower rates of responding can be sustained yet still achieve the same pharmacological actions as lower drug doses, can contribute to the descending arm of the curve (Fowler and Kenny, 2011). Similarly, disruption of behavioral performance also contributes to decreased responding when higher drug doses are self-administered (Fowler and Kenny, 2011). Obtaining the rewarding effects of nicotine while avoiding its aversive and performance-disrupting effects is thought to play a major role in determining patterns and amounts of tobacco intake in smokers (Fowler and Kenny, 2014). Moreover, nicotine-induced adaptive responses in the brain systems that regulate nicotine reward and aversion likely regulate the establishment and maintenance of the tobacco habit with genetic variation that influences these processes, rendering individuals more or less sensitive to the abuse liability of tobacco products (Jensen et al., 2015). Neuronal nicotinic acetylcholine receptors (nAChRs) are the major substrates in the brain for the rewarding and aversive actions of nicotine. The precise molecular, cellular, and circuit-level mechanisms through which different nAChR subtypes regulate these properties of nicotine are not yet fully characterized, but important new insights into these processes have emerged in recent years. Here, we summarize some of the most recent findings on the mechanisms of nicotine reward and aversion and the role for $\mathrm{nAChR}$ subtypes in these processes.

\section{B. Structural Architecture of $n A C h R s$}

Neuronal nAChRs are comprised of five distinct membrane-spanning subunits $(\alpha$ and $\beta$ ) that combine to form a functionally mature pentameric receptor complex (Hucho and Changeux, 1973; Deneris et al., 1991; Sargent, 1993; Albuquerque et al., 1995; Lena and Changeux, 1998). The mature receptor pentamer functions as an allosteric complex that can assume inactive, active, or desensitized confirmational states (Changeux and Taly, 2008), with the cognate agonist acetylcholine or other agonists, such as nicotine, stabilizing the active confirmation associated with an open inner transmembrane cationic channel (Fig. 1). Nicotine and other exogenous agonists can also drive nAChRs into a desensitized state (Changeux, 1979; Changeux et al., 1984) with the propensity to enter and exit desensitization related to the subunit composition of the receptor complex and influenced by 


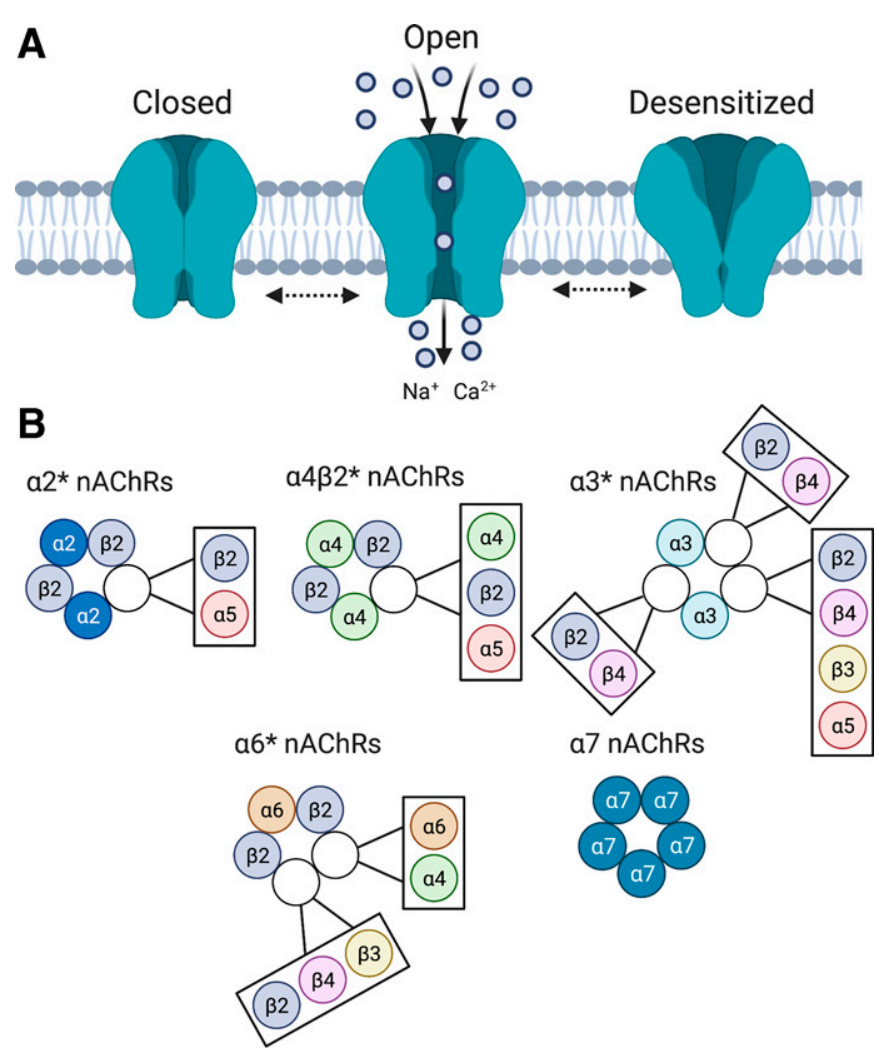

Fig. 1. Structural organization nicotinic acetylcholine receptors. (A) $\mathrm{nAChRs}$ are pentameric ligand-gated cationic channels. nAChR agonists, such as acetylcholine and nicotine, stabilize the receptor in the active confirmation associated with an open transmembrane pore permeable to calcium sodium $\left(\mathrm{Na}^{+}\right)$, potassium $\left(\mathrm{K}^{+}\right)$, and $\left(\mathrm{Ca}^{2+}\right)$ ions. Prolonged stimulation of the receptor by agonists, such as nicotine, can drive the receptor into an inactive "desensitized" state. (B) Major stoichiometries of homopentameric or heteropentameric nAChRs expressed in the mammalian brain. The open white circles indicate that one of the adjacent subunits contained within the adjacent box are often incorporated into that $n A C h R$ subtype.

various post-translational modifications to this complex. The neuronal $\alpha$ subunit exists in nine isoforms $(\alpha 2-\alpha 10)$, whereas the neuronal $\beta$ subunit exists in three isoforms ( $\beta 2-\beta 4$ ) (Elgoyhen et al., 1994, 2001; Le Novere et al., 2002). Assembly of nAChR subunits into a mature receptor is a tightly regulated process that requires appropriate subunit interactions, with only certain subunit stoichiometries able to generate functional nAChRs. The molecular mechanisms that control assembly of nAChR subunits into mature receptor complexes are poorly understood (Gotti et al., 2009), but modern genome-wide screening approaches are beginning to reveal the cellular components and processes involved (Gu et al., 2019). The major stoichiometries of nAChRs found in the mammalian brain are summarized in Fig. 1. Briefly, homomeric $\mathrm{nAChRs}$ are comprised of five $\alpha 7$, $\alpha 8$, or $\alpha 9$ subunits, whereas heteromeric nAChRs contain both $\alpha$ and $\beta$ subunits. Heteromeric nAChRs contain agonist binding sites at the interface between an $\alpha$ and $\beta$ subunit (Changeux, 1979; Changeux et al., 1984; Gotti et al., 2007). Nicotinic receptors containing $\alpha 4$ and $\beta 2$ subunits are denoted as $\alpha 4 \beta 2 * \mathrm{nAChRs}$, with the asterisk signifying that the $\mathrm{nAChR}$ contains other unidentified subunits. $\alpha 4 \beta 2^{*} \mathrm{nAChRs}$ are the most abundant subtype in the mammalian central nervous system (CNS) and account for the high-affinity nicotine binding sites in the brain (Flores et al., 1992). Nicotinic receptors containing $\alpha 3$ and $\beta 4$ subunits $\left(\alpha 3 \beta 4^{*}\right.$ nAChRs) are abundantly expressed by neurons in the autonomic nervous system and are sometimes referred to as the "ganglionic" nAChRs (Kemp and Morley, 1986). $\alpha 5$ subunits do not contain an agonist binding domain and thus do not form functional homomeric channels or heteromeric channels when coexpressed with $\beta 2$ or $\beta 4$ nAChR subunits and instead serve as "accessories" that modify the pharmacology of the receptor complexes into which they incorporate (Ramirez-Latorre et al., 1996; Gotti et al., 2009). $\beta 3$ also functions as an accessory subunit (Gotti et al., 2009) and plays a particularly important role in facilitating the assembly and function of $\alpha 6^{*}$ nAChRs. The role of $n A C h R$ subtypes comprised of these different subunits in regulating the behavioral actions of nicotine is reviewed below.

\section{Dopamine Mechanisms of Nicotine Reward}

\section{A. nAChRs in Brain Dopamine Systems}

nAChRs in the CNS are located primarily on presynaptic terminals (Wonnacott, 1997) but can also be found at somatodendritic and postsynaptic locations (Clarke et al. 1986; Sargent, 1993). Atypically, nAChRs are also found on the axons of medial habenula $(\mathrm{mHb})$ neurons that comprise the fasciculus retroflexus tract (Clarke et al., 1986; Mulle et al., 1991; Grady et al., 2009; Passlick et al., 2018). The major function of nAChRs in the CNS is the modulation of neurotransmitter release. Accordingly, nicotine stimulates the release of many transmitters in addiction-relevant regions of the brain and enhances neurotransmission in ventral tegmental area (VTA) and nucleus accumbens (NAc), which have been heavily implicated in nicotine reinforcement processes (Schwartz et al., 1984; Carboni et al., 2000b; Mansvelder and McGehee, 2000; Fu et al., 2003). nAChRs are expressed by midbrain dopamine neurons (Clarke and Pert, 1985), and what it has in common with other major drugs of abuse nicotine is that is enhances dopamine release in the NAc of rodents and human smokers (Imperato et al., 1986; Di Chiara and Imperato, 1988; Marshall et al., 1997; Johnson et al., 2000; Brody et al., 2004, 2006, 2009). This action is thought to reflect the ability of nicotine to stimulate VTA dopamine neurons (Pidoplichko et al., 1997) and increase their burst patterns of firing (Grenhoff et al., 1986; De Biasi and Dani, 2011). The stimulatory actions of nicotine on mesoaccumbens dopamine transmission were thought to exclusively reflect its actions at 
nAChRs located in the VTA (Nisell et al., 1994a,b). However, recent findings have highlighted the contributions of nAChRs expressed on the terminals of dopamine neurons in the striatum (Schwartz et al., 1984; Rice and Cragg, 2004; Cachope et al., 2012; Threlfell et al., 2012). As reviewed in detail below, the majority of studies investigating the mechanisms of nicotine reward have focused on the role of mesoaccumbens dopamine neurons in this process. However, it is important to note that nicotine elicits rewarding effects through actions at nAChRs located outside the mesocorticolimbic dopamine system. Indeed, nicotine is self-administered directly into the central linear nucleus and supramammillary nucleus by rats (Ikemoto et al., 2006), yet the mechanisms underlying the reinforcing actions in these sites and the link between these sites and the stimulatory effects of nicotine on VTA dopamine neurons have not been explored. The rewarding effects of nicotine are also blunted in genetically modified mice in which genes encoding $\mu$ opioid receptors, opioid peptides, or other related neuropeptide genes have been null mutated, hinting at dopamine-independent mechanisms of nicotine reward (Berrendero et al., 2002, 2005; Walters et al., 2005; Galeote et al., 2009; Sakoori and Murphy, 2009; Trigo et al., 2009; Neugebauer et al., 2011). Nevertheless, remarkably little is known about the role of nAChRs in dopamine-independent mechanisms of nicotine reward.

Nicotine enhances dopamine transmission more robustly in the shell region of the NAc compared with the NAc core region (Nisell et al., 1997; Lecca et al., 2006), with the NAc shell thought to play an important role in nicotine reward (Iyaniwura et al., 2001; Sellings et al., 2008). Dopamine receptor antagonists block the lowering effects of nicotine on intracranial self-stimulation (ICSS) thresholds (Huston-Lyons et al., 1993; Ivanova and Greenshaw, 1997), which reflects the direct stimulatory effects of nicotine on brain reward systems (Wise et al., 1992). Lesioning of midbrain dopamine neurons using the toxin 6-hydroxydopamine abolished the locomotor-stimulating properties of nicotine (Clarke et al., 1988) and reduced intravenous nicotine self-administration in rats (Corrigall et al., 1992; Singer and Wallace, 1984). Blockade of dopamine receptors also reduced nicotine self-administration in rats (Corrigall and Coen, 1991; Corrigall et al., 1992) and attenuated nicotine-induced conditioned place preference (CPP) (Acquas et al., 1989; Spina et al., 2006). These findings suggest that dopamine transmission plays a key role in the motivational properties of nicotine. Notably, however, dopamine receptor and nAChR antagonists increased tobacco consumption in human cigarette smokers (Nemeth-Coslett et al., 1986; Dawe et al., 1995), which is opposite to the effects of such manipulations on nicotine self-administration in rats (Watkins et al., 1999). The reason for this discrepancy is unclear but likely reflects the fact that rodents that self-administer nicotine for a few weeks are less dependent on nicotine than smokers who have been exposed to nicotine for many months or years. Whatever the underlying mechanisms, these findings support an important role for midbrain dopamine neurons, and the nAChRs that regulate mesoaccumbens dopamine transmission, in regulating the motivational properties of nicotine that drive the tobacco habit in human smokers (Corrigall et al., 1992; Fu et al., 2000; Grillner and Svensson, 2000; Tapper et al., 2004; David et al., 2006; Ikemoto et al., 2006).

\section{B. $\beta 2^{*} n A C h R$ Subtypes and Nicotine Reward}

$\alpha 4 \beta 2 *$ nAChRs are the most abundant subtype in the mammalian CNS (Flores et al., 1992). $\alpha 4 \beta 2 \mathrm{nAChRs}$ occur in two discrete stoichiometries: $(\alpha 4 \beta 2)_{2} \beta 2$ or $(\alpha 4 \beta 2)_{2} \alpha 4$ subtypes (Nelson et al., 2003; Gotti et al., 2009). $(\alpha 4 \beta 2)_{2} \beta 2 \mathrm{nAChRs}$ are far more sensitive to agonist-induced activation ( $\mathrm{EC}_{50} \sim 1 \mathrm{mM}$ for acetylcholine) than the $(\alpha 4 \beta 2)_{2} \alpha 4$ subtype $\left(\mathrm{EC}_{50} \sim 100 \mathrm{mM}\right.$ ) (Gotti et al., 2009). However, $(\alpha 4 \beta 2)_{2} \beta 2 \mathrm{nAChRs}$ are also far more sensitive to agonist-induced desensitization than $(\alpha 4 \beta 2)_{2} \alpha 4 \mathrm{nAChRs}$ (Gotti et al., 2009). In addition to $(\alpha 4 \beta 2)_{2}(\alpha 4)$ and $(\alpha 4 \beta 2)_{2}(\beta 2) \mathrm{nAChRs}$, several other $\beta 2^{*}$ nAChR stoichiometries exist in the brain and are involved in regulating behavioral responses to nicotine. For example, $\beta 2 *$ nAChRs can incorporate $\alpha 2$ or $\alpha 6$ subunits in some regions of the brain (Zoli et al., 2002; Salminen et al., 2004; Grady et al., 2009; Gotti et al., 2010). Likewise, $\beta 4^{*}$ nAChRs can also incorporate $\alpha 2$ and $\alpha 6$ subunits (Zoli et al., 2002; Salminen et al., 2004; Gotti et al., 2009; Grady et al., 2009; Azam et al., 2010; Dash and Li, 2014). Using polymerase chain reactions (PCRs) to assess nAChR subunit expression in animals after unilateral lesion of VTA dopamine neurons, it was found that mRNA transcripts for $\alpha 2, \alpha 3, \alpha 5, \alpha 6, \alpha 7$ and $\beta 4$ subunits were downregulated in the lesioned hemisphere compared with the intact side (Charpantier et al., 1998). By contrast, mRNA for $\alpha 4, \beta 2$ and $\beta 3$ subunits was detected after the lesion of dopamine neurons (Charpantier et al., 1998). This suggests that $\alpha 2, \alpha 3, \alpha 5$, $\alpha 6, \alpha 7$, and $\beta 4 \mathrm{nAChR}$ subunits are expressed by VTA dopamine neurons that project to the NAc, whereas $\alpha 4$, $\beta 2$, and $\beta 3$ subunits are expressed by nondopamine cells in the VTA. Using PCR and single-cell electrophysiological recordings, almost $100 \%$ of dopamine and nondopamine neurons in the VTA were shown to express mRNA for the $\alpha 4$ subunit (Klink et al., 2001), whereas $\sim 90 \%$ of dopamine neurons and $20 \%$ of GABA neurons expressed $\beta 3 \mathrm{nAChR}$ subunits (Klink et al., 2001). Further, $70 \%-75 \%$ of VTA dopamine neurons expressed $\alpha 5$ and $\alpha 6$ subunit mRNAs, but a much lower proportion of GABAergic cells (10\%-20\%) expressed these subunit transcripts (Klink et al., 2001). A similar proportion of VTA dopamine and GABA neurons $(\sim 40 \%)$ expressed $\alpha 7 \mathrm{nAChR}$ subunit transcripts (Klink et al., 2001). $\beta 4$ mRNA was detected only at low concentrations in VTA 
cells. Based on these and related findings, it was proposed that three major subtypes of nAChRs are expressed by VTA dopamine neurons: $(\alpha 4 \beta 2)(\alpha 6 \beta 2)(\alpha 5)$, $(\alpha 4 \beta 2)_{2}(\alpha 5)$, and $\alpha 7$ (Fig. 2). On VTA GABAergic neurons, it was proposed that two nAChR subtypes predominate: $(\alpha 4 \beta 2)_{2}(\alpha 4)$ and $\alpha 7 \mathrm{nAChRs}$ (Fig. 2). Notably, $\beta 3^{*} \mathrm{nAChRs}$ are not thought to exist in the VTA despite the high concentrations of $\beta 3$ mRNA expressed by dopamine neurons (Klink et al., 2001). This is because $\beta 3$ subunits are transported to the terminal regions to which VTA neurons project (Forsayeth and Kobrin, 1997), with high $\beta 3$ protein concentrations detected in striatum but not VTA (Forsayeth and Kobrin, 1997; Reuben et al., 2000; Salminen et al., 2004). Hence, $\beta 3 \mathrm{nAChR}$ subunits are likely to be incorporated exclusively into the nAChRs on the terminals of VTA neurons in the striatum and elsewhere in the brain, where they regulate the stimulatory effects of nicotine on dopamine transmission (Klink et al., 2001). Immunoprecipitation, ligand-binding, genetic deletion, and targeted lesion studies support the existence of at least four species of nAChRs on the terminals of VTA dopamine neurons in striatum: $(\alpha 4 \beta 2)_{2}(\alpha 4)$, $(\alpha 4 \beta 2) 2(\alpha 5), \quad(\alpha 4 \beta 2)(\alpha 6 \beta 2) \beta 3, \quad(\alpha 6 \beta 2)_{2} \beta 2$, and $(\alpha 6 \beta 2)_{2} \beta 3$ (Zoli et al., 2002; Salminen et al., 2004) (Fig. 2). In addition to dopamine neurons, GABA neurons in the VTA also express $\alpha 6 \beta 2^{*}$ nAChRs (Yang et al., 2011). There is evidence that $\alpha 6$ can occasionally assemble into $\beta 4^{*}$

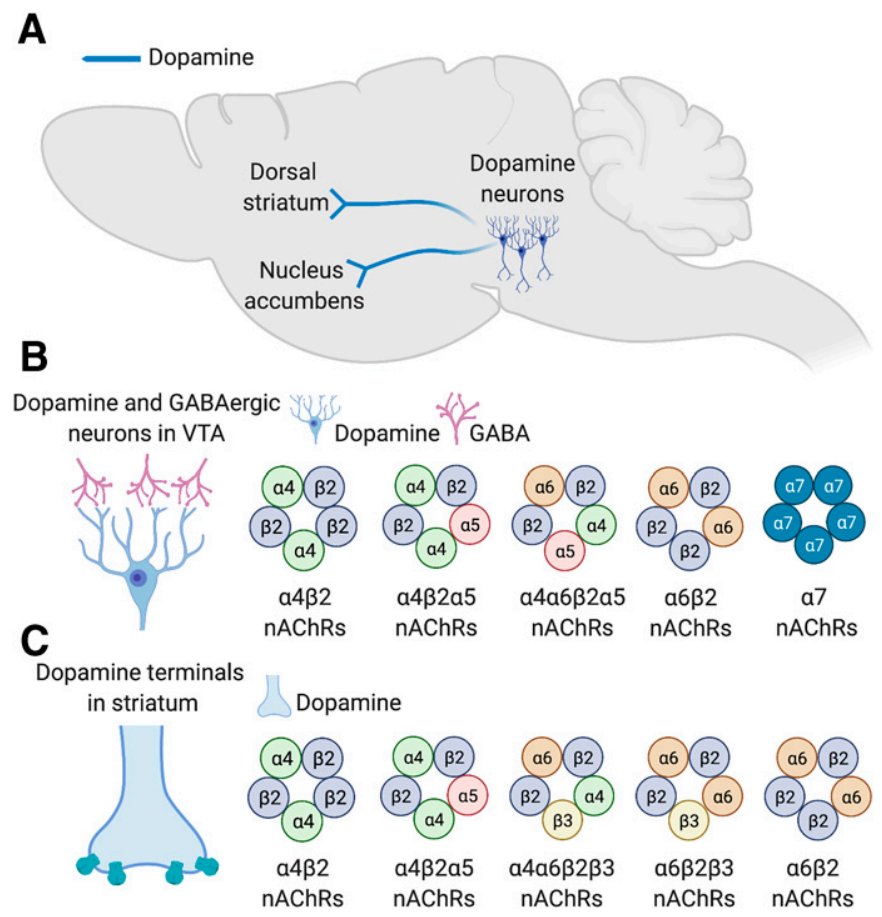

Fig. 2. nAChR subtypes in brain dopamine systems. (A) Ventral midbrain dopaminergic neurons that project to the dorsal striatum or nucleus accumbens are stimulated by nicotine, resulting in increased dopamine transmission in the striatum. (B) Major stoichiometries of nAChRs predicted to be expressed by dopaminergic and GABAergic neurons in the ventral tegmental area. (C) Major stoichiometries of nAChRs predicted to be expressed by on the terminals of dopaminergic in the dorsal striatum and nucleus accumbens.
$\mathrm{nAChRs}$, with $\alpha 6 \beta 4^{*} \mathrm{nAChRs}$ involved in regulating norepinephrine release in the hippocampus (Azam et al., 2010) (Fig. 2). However, $\alpha 6 \beta 4^{*}$ are thought to be minimally involved in regulating mesoaccumbens dopamine transmission (Azam et al., 2010). It has been speculated that each dopamine neuron in the VTA expresses only one particular nAChR subtype, meaning that these cells can be functionally categorized based on their nAChR expression patterns, with the particular nAChR subtype expressed by each class of dopamine neuron determining their responses to nicotine (Yang et al., 2009).

$\alpha 4 \beta 2 *$ nAChRs are the major subtype responsible for the stimulatory effects of nicotine on midbrain dopamine neurons (Subramaniyan and Dani, 2015; Thiruchselvam et al., 2017). Nicotine-induced burst firing of midbrain dopamine neurons is abolished in mice lacking the $\beta 2$ nAChR subunit (Mameli-Engvall et al., 2006), resulting in attenuated striatal dopamine release in response to nicotine treatment (Picciotto et al., 1998). Accordingly, $\beta 2$ subunit knockout mice are almost entirely insensitive to the reinforcing properties of nicotine (Picciotto et al., 1998), with this effect attributed to the diminished stimulatory actions of nicotine on VTA dopamine neurons (Besson et al., 2006; Pons et al., 2008; Orejarena et al., 2012; Grieder et al., 2019; Bagdas et al., 2019). Nicotine stimulates VTA dopamine neurons via $\beta 2^{*}$ nAChRs located directly on these cells (Durand-de Cuttoli et al., 2018). Nicotine also stimulates populations of $\beta 2^{*}$ and $\alpha 7 \mathrm{nAChRs}$ on the terminals of glutamatergic inputs to VTA dopamine neurons, resulting in prolonged increases in excitatory transmission in the VTA (Yan et al., 2018; Mansvelder and McGehee, 2000). In parallel, nicotine activates then rapidly desensitizes $\beta 2 *$ nAChRs expressed by VTA GABAergic neurons (Mansvelder et al., 2002; Yang et al., 2011). These findings suggest that the net action of nicotine is an increase in the ratio of excitatory to inhibitory transmission onto VTA dopamine neurons (Durandde Cuttoli et al., 2018), with $\beta 2 *$ nAChRs critical for these actions. Indeed, virus-mediated re-expression of the Chrnb2 gene, which encodes the $\beta 2 \mathrm{nAChR}$ subunit, in the VTA of $\beta 2 \mathrm{nAChR}$ subunit knockout mice restored the ability of nicotine to evoke burst firing of dopamine neurons in these animals (Maskos et al., 2005; Mameli-Engvall et al., 2006; Naude et al., 2016). Chrnb2 re-expression in the VTA also restored the reinforcing and locomotor-stimulating properties of nicotine in $\beta 2$ knockout mice (Maskos et al., 2005). However, a more recent study showed that virus-mediated Chrnb2 re-expression in the midbrain dopamine system of $\beta 2$ knockout mice did not reinstate their sensitivity to nicotine in a CPP procedure but did reinstate sensitivity to the locomotor-stimulating effects of nicotine (Avale et al., 2008; Mineur et al., 2009). These findings could reflect different components of the midbrain dopamine system being involved in regulating 
discrete behavioral responses to nicotine, with these components differentially targeted across studies. Alternatively, it is possible that discrete populations of cells within the VTA regulate behavioral responses to nicotine, with the virus vectors or other factors used across different studies differentially impacting these cell populations. Consistent with both possibilities, nicotine is self-administered only into posterior but not anterior regions of VTA by rats (Ikemoto et al., 2006), and nicotine modulates the balance between excitatory and inhibitory drive onto VTA dopamine neurons in a manner that depends on their precise location within the VTA (Yan et al., 2019). Furthermore, Chrnb2 re-expression concurrently in both VTA dopamine and GABAergic neurons was required to rescue the sensitivity of $\beta 2$ subunit knockout mice to the actions of nicotine in CPP and self-administration procedures (Tolu et al., 2013; Grieder et al., 2019). Hence, rewardrelated behavioral responses to nicotine are likely parsed into discrete anatomic regions of the VTA, and within these regions many different classes of neurons are likely to participate. This concept of spatial and cellular segregation of nicotine reward within the VTA is considered in more detail below.

Systemic or intra-VTA administration of the $\beta 2^{*}$ $\mathrm{nAChR}$ antagonist dihydro- $\beta$-erythroidine ( $\mathrm{DH} \beta \mathrm{E})$ decreased nicotine self-administration in rats (Corrigall and Coen, 1989; Watkins et al., 1999). In addition, $\mathrm{DH} \beta \mathrm{E}$ reduced the stimulatory effects of nicotine on brain reward systems, as shown by attenuated nicotineinduced lowering of ICSS thresholds in rats (Ivanova and Greenshaw, 1997; Harrison et al., 2002). As DH $\beta \mathrm{E}$ is relatively selective for $\beta 2^{*} \mathrm{nAChRs}$ compared with other classes of nAChRs (Harvey and Luetje, 1996; Harvey et al., 1996), these findings further implicate this nAChR subtype in the motivational properties of nicotine. The novel nAChR compound SSR591813, considered a partial agonist at $\alpha 4 \beta 2^{*}$ nAChRs, decreased nicotine self-administration in rats (Cohen et al., 2003). Similarly, the novel nAChR compound UCI-30002, a partial agonist at $\alpha 4 \beta 2 * \mathrm{nAChRs}$, also decreased nicotine self-administration in rats (Yoshimura et al., 2007), whereas 5-iodo-A-85380, a putative agonist at $\beta 2 *$ nAChRs, was actively self-administered by rats (Liu et al., 2003). Varenicline is a partial agonist at $\alpha 4 \beta 2 *$ nAChRs but a full agonist at $\alpha 7 \mathrm{nAChRs}$ (Coe et al., 2005; Mihalak et al., 2006; Lerman et al., 2007; Reus et al., 2007; Dwoskin et al., 2009) and can compete with nicotine for binding sites on $\alpha 4 \beta 2 *$ nAChRs in the VTA and elsewhere in the brain. In this manner, varenicline can attenuate the stimulatory action of nicotine on dopamine transmission (Coe et al., 2005; Reperant et al., 2010). Varenicline dose-dependently decreased nicotine self-administration in rats (Rollema et al., 2007; O'Connor et al., 2010), with this action thought to directly reflect the ability of varenicline to attenuate the stimulatory actions of nicotine on VTA dopamine neurons (Harmey et al., 2012). Cytisine and dianicline are both structurally related to varenicline and similarly act as partial agonists at $\alpha 4 \beta 2^{*}$ nAChRs (Coe et al., 2005). These compounds have shown clinical utility as smoking cessation medications in humans (Barlow and McLeod, 1969; Reavill et al., 1990; Etter, 2006; Rollema et al., 2010). Bupropion has been shown to antagonize $\alpha 4 \beta 2 *$ nAChRs (Alkondon and Albuquerque, 2005), whereas nicotine replacement therapy is thought to facilitate smoking cessation by stimulation $\alpha 4 \beta 2 *$ nAChRs in the midbrain (Harmey et al., 2012). Hence, currently available smoking cessation therapeutics have at least some actions at $\alpha 4 \beta 2^{*} \mathrm{nAChRs}$. Together, these findings support a major role for $\alpha 4 \beta 2^{*} \mathrm{nAChRs}$ located in midbrain dopamine systems in regulating the reinforcing properties of nicotine.

Nicotine-induced upregulation of $\mathrm{nAChR}$ expression has been detected in the brains of rodents and human smokers (Marks et al., 1983; Breese et al., 1997; Staley et al., 2006; Nashmi et al., 2007). nAChR upregulation is thought to modify the subsequent actions of nicotine on brain reward systems and thereby contribute to the development of nicotine dependence (Esterlis et al., $2014,2016)$. Indeed, $\beta 2 * \mathrm{nAChRs}$ expressed by VTA GABAergic neurons are highly sensitive to nicotineinduced upregulation, with this effect correlated with increased sensitivity to the rewarding properties of the drug (Nashmi et al., 2007; Ngolab et al., 2015). Positron emission tomographic imaging of the brains of smokers using a radiotracer to measure densities of $\alpha 4 \beta 2^{*}$ nAChRs has shown that greater levels of $\mathrm{nAChR}$ upregulation are associated with greater difficulty in achieving and maintaining abstinence from tobacco use (Brody et al., 2014). The fact that $\beta 2 *$ nAChRs expressed by VTA GABAergic neurons are so sensitive to nicotine-induced upregulation suggests that adaptive responses in VTA GABAergic transmission may play a particularly important role in the development of nicotine dependence. Intriguingly, farnesol, which is often added to nicotine contained in electronic delivery systems to improve its flavor, can increase the activity of VTA dopamine neuron while decreasing the activity of local GABAergic neurons in a manner similar to nicotine (Avelar et al., 2019). Menthol, which is also incorporated into cigarettes to modify their flavor (Ai et al., 2016), also modifies the function of $\beta 2^{*}$ nAChRs in the VTA to enhance the rewarding effects of nicotine (Henderson et al., 2017). This suggests that flavorants added to cigarettes and electronic smoking devices may facilitate nicotine use not only by masking the noxious bitter taste of nicotine but by directly modifying the receptor and cellular substrates in the VTA upon which nicotine acts.

\section{C. $\alpha 4^{*} n A C h R$ Subtypes and Nicotine Reward}

The most common stoichiometry of $\beta 2 *$ heteromeric $\mathrm{nAChRs}$ is thought to incorporate two $\alpha 4$ and three $\beta 2$ 
subunits, which is denoted as $(\alpha 4 \beta 2)_{2}(\beta 2)$, and contains orthosteric binding sites for acetylcholine, nicotine, and other agonists at the two $\alpha 4 / \beta 2$ interfaces (Zwart and Vijverberg, 1998; Albuquerque et al., 2009). In a less common stoichiometry denoted as $(\alpha 4 \beta 2)_{2}(\alpha 4)$, one of the $\beta 2$ subunits is substituted with an $\alpha 4$ subunit (Zwart and Vijverberg, 1998; Mazzaferro et al., 2011). This incorporates a third "unorthodox" agonist binding site at the $\alpha 4 / \alpha 4$ interface (Mazzaferro et al., 2011). As noted above, the $(\alpha 4 \beta 2)_{2}(\alpha 4)$ nAChR stoichiometry has lower affinity for nicotine than the $(\alpha 4 \beta 2)_{2}(\beta 2)$ subtype (Moroni et al., 2006; Campling et al., 2013). However, the unorthodox binding site in the $(\alpha 4 \beta 2)_{2}(\alpha 4) \mathrm{nAChR}$ stoichiometry results in 3 - to 4 -fold greater levels of receptor activation by acetylcholine or nicotine when compared with the $(\alpha 4 \beta 2)_{2}(\beta 2)$ stoichiometry (Moroni et al., 2006; Timmermann et al., 2012; Wang et al., 2015; Jain et al., 2016). Hence, the $(\alpha 4 \beta 2)_{2}(\beta 2)$ and $(\alpha 4 \beta 2)_{2}(\alpha 4)$ subtypes can be considered "high-affinity" and "high-efficacy" nAChRs, respectively. Both of these nAChR stoichiometries are thought to be expressed by neurons in the adult mammalian brain (Marks et al., 2007; Gotti et al., 2008), and pharmacological agents have been identified or developed that can discriminate between them (Moroni et al., 2006). The smoking-cessation therapeutics varenicline and cytisine are partial agonists at $(\alpha 4 \beta 2)_{2}(\alpha 4)$ $\mathrm{nAChRs}$ but are inactive at $(\alpha 4 \beta 2)_{2}(\beta 2) \mathrm{nAChRs}$ (Moroni et al., 2006; Campling et al., 2013). In addition, the $(\alpha 4 \beta 2)_{2}(\alpha 4) \mathrm{nAChR}$ positive allosteric modulator NS9283 (Mazzaferro et al., 2019) decreased nicotine self-administration in rats (Maurer et al., 2017). NS9283, cytisine, and varenicline also decreased ethanol intake in rats (Steensland et al., 2007; Bell et al., 2009; SotomayorZarate et al., 2013; Wang et al., 2020). Hence, $(\alpha 4 \beta 2)_{2}(\alpha 4)$ nAChRs likely play an important role in the reinforcing properties of nicotine and other drugs of abuse, and compounds that modulate this nAChR stoichiometry may serve as novel therapeutics for substance use disorders (see Fig. 2).

Similar to $\beta 2$ nAChR subunit knockout mice (Walters et al., 2006), deletion of $\alpha 4 \mathrm{nAChR}$ subunits in the ventral midbrain blocked nicotine-induced CPP in mice (Peng et al., 2017). Conditional deletion of $\alpha 4$ nAChR subunits from dopamine neurons in mice similarly blocked nicotine-induced CPP (McGranahan et al., 2011). Conversely, mutant mice expressing a hypersensitive $\alpha 4 \mathrm{nAChR}$ subunit are hyper-responsive to nicotine reward, as reflected by the establishment of CPP for very low doses of nicotine that have no detectable effects in wild-type mice (Tapper et al., 2004). The hypersensitive $\alpha 4$ mutant mice also showed increased sensitivity to the stimulatory effects of nicotine on midbrain dopamine neurons (Tapper et al., 2004). Using a behavioral procedure in which nicotine is self-administered via the tail vein during a single session, it has been shown that $\beta 2$ and $\alpha 4$ subunit knockout mice but not $\alpha 7$ knockout mice consume markedly less nicotine than their wild-type counterparts (Pons et al., 2008). Lentivirus-mediated re-expression of the $\beta 2$ or $\alpha 4$ subunit genes in the VTA but not substantia nigra of the respective knockout mice "rescued" their nicotine intake in this acute self-administration procedure (Pons et al., 2008). However, $\alpha 4 \mathrm{nAChR}$ subunit knockout mice did not show any difference in nicotine intake using a more traditional chronic intravenous (jugular catheter) self-administration procedure (Cahir et al., 2011). However, they did show attenuated locomotor suppression in response to injection of a relatively high dose of nicotine (Cahir et al., 2011), a behavioral response thought to reflect the aversive actions of nicotine (Morrison and Stephenson, 1972; Stolerman et al., 1973; Clarke and Kumar, 1983a,b; Hentall and Gollapudi, 1995; Salas et al., 2004a; Frahm et al., 2015; Antolin-Fontes et al., 2020). Hence, it is possible that $\alpha 4^{*}$ $\mathrm{nAChRs}$ play a more prominent role in nicotine aversion than nicotine reward at least under the testing conditions in these experiments (discussed in more detail below) (see Fig. 2).

\section{D. $\alpha 6^{*} n A C h R$ Subtypes and Nicotine Reward}

Much interest has centered on the potential involvement of $\alpha 6^{*}$ nAChR subunits in nicotine reward processes. This interest has arisen in large part because of the high concentrations and restricted patterns of expression of mRNA transcripts for $\alpha 6$ subunits within the VTA and other catecholaminergic nuclei of the brain (Le Novere et al., 1996; Quik et al., 2000; Azam et al., 2002; Champtiaux et al., 2002; Gotti et al., 2006a). Non$\alpha 4(\alpha 6 \beta 2)_{2}{ }^{*}$ nAChRs are abundantly expressed by mesoaccumbens dopamine neurons, whereas $(\alpha 6 \beta 2)(\alpha 4 \beta 2)^{*}$ nAChRs are expressed by nigrostriatal dopamine neurons (Gotti et al., 2010). The stimulatory effects of nicotine on VTA dopamine neurons were attenuated $\alpha 6$ nAChR subunit knockout mice (Liu et al., 2012). $\alpha 6$ nAChR subunits were also upregulated in rats by chronic intravenous nicotine self-administration (Parker et al., 2004) in both dopaminergic and GABAergic neurons in the VTA (Akers et al., 2020). The magnitude by which $(\alpha 6 \beta 2)(\alpha 4 \beta 2)^{*}$ nAChRs were upregulated in the VTA of mice corresponded to their sensitivity to the rewarding effects of nicotine (Akers et al., 2020). However, it was previously reported that $\alpha 6^{*} \mathrm{nAChRs}$ were downregulated by chronic nonvolitional nicotine treatment through a process influenced by the presence or absence of $\beta 3 \mathrm{nAChR}$ subunits (Lai et al., 2005; Mugnaini et al., 2006; Perry et al., 2007; Marks et al., 2014). Hence, the volitional nature of nicotine delivery and their precise subunit composition likely determine whether $\alpha 6^{*}$ nAChRs are upregulated or downregulated by nicotine. $\alpha 6 \beta 2^{*} \mathrm{nAChRs}$ are activated by cytisine and varenicline far more efficiently than other $\beta 2^{*}$ nAChRs (Salminen et al., 2004; Bordia et al., 2012), suggesting that they may contribute to the clinical utility 
of these compounds as smoking-cessation therapeutics. Furthermore, allelic variation in the CHRNB3-CHRNA6 gene cluster on chromosome 8 , which encodes the $\beta 3$ and $\alpha 6 \mathrm{nAChR}$ subunits, respectively, increases vulnerability to tobacco dependence (Bierut et al., 2007; Thorgeirsson et al., 2010; Wen et al., 2016). Behavioral data have accumulated to support a role for $\alpha 6^{*} \mathrm{nAChRs}$ in nicotine reinforcement, but their involvement is complex, and their precise contributions remain unclear. $\alpha 6 \mathrm{nAChR}$ subunit knockout mice did not drink a nicotine-containing solution in a two-bottle choice procedure (Bagdas et al., 2019). Similarly, $\alpha 6 \mathrm{nAChR}$ subunit knockout mice do not self-administer nicotine using an acute tail-vein procedure during a single session (Pons et al., 2008). Lentivirusmediated re-expression of the $\alpha 6$ subunit in the VTA of the knockout mice re-established their sensitivity to nicotine reinforcement in this procedure (Pons et al., 2008). Transgenic mice expressing a gain-of-function $\alpha 6 \mathrm{nAChR}$ mutant subunit showed enhanced sensitivity to the stimulatory effects of nicotine on striatal dopamine transmission and increased locomotor stimulant responses to nicotine (Drenan et al., 2010), with these effects attributed to increased function of $(\alpha 6 \beta 2)(\alpha 4 \beta 2)^{*}$ nAChRs (Drenan et al., 2010; Engle et al., 2013). Pharmacological blockade of $\alpha 6^{*}$ nAChRs in the VTA or NAc of rodents abolished the stimulatory effects of nicotine on dopamine transmission in the striatum and decreased nicotine self-administration behavior (Brunzell et al., 2010; Gotti et al., 2010; Brunzell, 2012; Sanjakdar et al., 2015), with the $\alpha 6 \beta 2 *$ nAChR subtype hypothesized to play a prominent role in these effects (Whiteaker et al., 2000; Marks et al., 2014). Indeed, the $(\alpha 4 \beta 2)(\alpha 6 \beta 2) \beta 3 \mathrm{nAChR}$ subtype has the highest sensitivity to nicotine of any native $\mathrm{nAChR}$ so far identified (Grady et al., 2007). In addition, the novel nAChR antagonist bPiDDB ( $N, N^{\prime}$-dodecane-1,12-diyl-bis-3-picolinium dibromide) dose-dependently decreased nicotine self-administration in rats and attenuated the locomotor-stimulating effects of acute and repeated nicotine treatment (Neugebauer et al., 2006). The related $\alpha 6^{*}$ antagonist ( $N, N$-decane-1,10-diyl-bis-3-picolinium diiodide) bPiDI decreased intravenous nicotine self-administration in wild-type mice and in mice carrying a mutation in the $\alpha 4 \mathrm{nAChR}$ subunit that renders it insensitive to mecamylamine and other nAChR antagonists (Madsen et al., 2015). Considering that $\alpha 4 \mathrm{nAChR}$ subunit knockout mice intravenously self-administer nicotine at similar levels to wild-type mice (Cahir et al., 2011 ), it was proposed that $\alpha 6 \beta 2 *$ nAChRs are likely to be the critical subtype that regulates the reinforcing properties of nicotine (Madsen et al., 2015); for review, see (Brunzell 2012).

However, recent findings have raised questions about the degree to which $\alpha 6^{*}$ nAChRs contribute to the reinforcing properties of nicotine. $\alpha 4^{*}$ but not $\alpha 6^{*}$ nAChRs regulate nicotine-induced bursting of VTA dopamine neurons (Exley et al., 2011). The novel $\alpha 6 \beta 2 *$ nAChR agonist TC299423 induced only modest rewarding effects in wild-type, which were enhanced in $\alpha 6$ gain-of-function mutant mice (Wall et al., 2017). However, TC299423 had no-effects, no-intravenousnicotine self-administration in rats (Wall et al., 2017). Using an intra-VTA self-administration procedure, it was shown that $\alpha 6 \mathrm{nAChR}$ subunit knockout mice will self-administer similar quantities of nicotine in the VTA as wild-type mice, whereas $\alpha 4$ subunit knockout mice self-administer far less nicotine (Exley et al., 2011). This suggests that $\alpha 6^{*}$ nAChRs located in the VTA are unlikely to regulate the reinforcing actions of nicotine. One explanation to reconcile these discrepant findings is that $\alpha 4^{*} \mathrm{nAChRs}$ may dominate the local actions of nicotine in the VTA, whereas $\alpha 6^{*}$ nAChRs located on the terminals of dopamine neurons may regulate the local actions of nicotine in the striatum (Exley et al., 2011). Indeed, both $\alpha 4^{*}$ and $\alpha 6^{*}$ contribute to the stimulatory effects of nicotine on dopamine release in the striatum (Cui et al., 2003; Salminen et al., 2004, 2007; Grady et al., 2007, 2010), with $\alpha 4 \beta 2^{*}$ and (non- $\left.\alpha 4\right)(\alpha 6 \beta 2)_{2} *$ nAChRs located on the terminals of dopamine neurons regulating the actions of nicotine in the striatum (Exley et al., 2008, 2011) (Fig. 2). If $\alpha 6^{*} \mathrm{nAChRs}$ only regulate the actions of nicotine in the striatum and not in the VTA, then nicotine may modulate dopamine release through dissociable actions on dopamine neurons at somatodendritic and terminal brain regions (Reuben et al., 2000). This raises important questions about the function and behavioral significance of dopamine released by nicotine acting at somatodendritic versus terminal locations (discussed in more detail below).

\section{E. $\beta 3^{*} n A C h R$ Subtypes and Nicotine Reward}

The $\beta 3 \mathrm{nAChR}$ subunit gene is located in the same genomic locus as the $\alpha 6$ subunit gene, and both are thought to be cotranscribed (Moen et al., 2021). Moreover, the $\beta 3$ subunit is known to facilitate the maturation and expression of $\alpha 6^{*}$ nAChRs (Gotti et al., 2006b, 2009; Drenan et al., 2008), and $\beta 3$ subunit knockout mice demonstrate markedly reduced $(\sim 75 \%$ lower) levels of $\alpha 6^{*} \mathrm{nAChRs}$ in the striatum compared with wild-type mice (Gotti et al., 2005). Hence, the $\beta 3$ nAChR subunit can be considered an accessory component of the $\alpha 6^{*}$ nAChRs that regulate striatal dopamine transmission (Fig. 2). The $\beta 3 \mathrm{nAChR}$ subunit has received considerable attention as a possible component of the $\mathrm{nAChR}$ subtypes that regulate nicotine reward processes. The $\mathrm{nAChR}$ antagonist $\alpha$-conotoxin MII partially inhibits nicotine-induced dopamine release from striatal synaptosomes (Kulak et al., 1997; Kaiser et al., 1998). $\alpha$-Conotoxin MII binding in the striatum was shown to depend on the expression of $\beta 3^{*}$ and $\alpha 6^{*}$ nAChRs (Champtiaux et al., 2002; Cui et al., 2003). This has led to the proposal that at least two populations of 
nAChRs regulate the stimulatory effects of nicotine on dopamine release in the striatum (Kulak et al., 1997) $\alpha$-conotoxin MII-sensitive and -insensitive components. Subsequent studies suggested that the $\alpha$-conotoxin MII-sensitive component of nicotine-evoked dopamine release in striatum requires $\beta 3^{*} \mathrm{nAChRs}$ that are partially dependent upon $\alpha 4$ subunits (Cui et al., 2003; Salminen et al., 2004), likely representing $\alpha 6 \beta 3 \beta 2^{*}$ and $\alpha 4 \alpha 6 \beta 3 \beta 2^{*}$ nAChRs. By contrast, the $\alpha$-conotoxin MII-insensitive component reflects the contributions of $\alpha 4^{*}$ nAChRs, likely representing $\alpha 4 \beta 2 *$ and $\alpha 4 \beta 2 \alpha 5^{*}$ nAChRs (Salminen et al., 2004). This is consistent with previous work described above, suggesting that mesoaccumbens dopamine neurons express four species of $\mathrm{nAChR}$ subtypes on their terminals, two of which contain $\beta 3$ subunits: $\alpha 6 \beta 3 \beta 2^{*}$ and $\alpha 4 \alpha 6 \beta 3 \beta 2^{*}$ nAChRs (Zoli et al., 2002).

\section{F. $\alpha 5^{*}$ nAChR Subtypes and Nicotine Reward}

Similar to $\beta 3, \alpha 5 \mathrm{nAChR}$ subunits do not reliably form functional $\mathrm{nAChRs}$ containing agonist binding sites when coexpressed with $\beta$ subunits (RamirezLatorre et al., 1996; Gerzanich et al., 1998; Kuryatov et al., 2008; Dash et al., 2012). Instead $\alpha 5$ nAChR subunits are thought to function as accessory subunits in mature nAChR complexes. In the adult mammalian brain, $\alpha 5$ subunits are thought to incorporate most efficiently into $\alpha 4 \beta 2^{*}$ nAChRs (Perry et al., 2007; Gotti et al., 2007; Kuryatov et al., 2008; Mao et al., 2008). Incorporation of an $\alpha 5$ subunit into $\alpha 4 \beta 2 * \mathrm{nAChRs}$ yields the $(\alpha 4 \beta 2)_{2} \alpha 5$ subtype that has the highest known permeability to calcium ions of any nAChR subtype (Ramirez-Latorre et al., 1996; Gotti et al., 2009); for recent review, see (Scholze and Huck, 2020). The presence of an $\alpha 5$ subunit can also alter receptor desensitization and upregulation dynamics in response to agonist exposure. In synaptosome and slice physiology preparations from mouse brain, up to 8-fold higher concentrations of nicotine and other agonists were required to desensitize $\alpha 4 \beta 2^{*}$ nAChRs that had incorporated an $\alpha 5$ subunit (Grady et al., 2012; Poorthuis et al., 2013; Wageman et al., 2014). Allelic variation in CHRNA5, the gene that encodes the $\alpha 5 \mathrm{nAChR}$ subunit, is heavily associated with vulnerability to nicotine dependence (Berrettini et al., 2008; Bierut et al., 2008; Hung et al., 2008; Thorgeirsson et al., 2008). In particular, the rs16969968 risk variant in CHRNA5 increases risk of tobacco dependence gives rise to an amino-acid substitution $(398 \mathrm{D} \rightarrow 398 \mathrm{~N})$ in the cytoplasmic domain in an amphipathic $\alpha$ helix just preceding the fourth transmembrane domain. This area of the subunit is known to influence channel permeability, particularly to $\mathrm{Ca}^{2+}$ ions (Wang et al., 1996, 1998; Gerzanich et al., 1998; Tapia et al., 2007; Kuryatov et al., 2008). This suggests that $\mathrm{nAChRs}$ that incorporate the mutant $\alpha 5 \mathrm{nAChR}$ have reduced function, which is consistent with experimental observations (Bierut et al., 2008). $\alpha 5 \mathrm{nAChR}$ subunits are expressed by midbrain dopamine neurons (Azam et al., 2002) and can incorporate into $\alpha 4 \beta 2^{*}$ (but not $\left.\alpha 6^{*}\right) \mathrm{nAChRs}$ to form a functional $(\alpha 4 \beta 2)_{2} \alpha 5 \mathrm{nAChR}$ subtype in these cells. In the VTA, $\alpha 5$ subunits are thought to facilitate the maturation and expression of $\alpha 4 \beta 2 \mathrm{nAChRs}$ and enhance their function (Chatterjee et al., 2013). As might be expected, incorporation of an $\alpha 5$ subunit also increases the resistance of $\alpha 4 \beta 2$ nAChRs in the VTA to agonist-induced desensitization (Chatterjee et al., 2013) and renders $\alpha 4 \beta 2 \alpha 5^{*}$ nAChRs in the ventral midbrain largely resistant to nicotineinduced upregulation (Mao et al., 2008).

The $(\alpha 4 \beta 2)_{2} \alpha 5 \mathrm{nAChR}$ subtype is expressed on the terminals of dopamine neurons in the striatum (Salminen et al., 2004; Mao et al., 2008; Grady et al., 2010; Scholze and Huck, 2020) (Fig. 2), where it serves as an important regulator of the stimulatory effects of nicotine on dopamine transmission. $(\alpha 4 \beta 2)_{2} \alpha 5 \mathrm{nAChRs}$ regulate dopamine release in the striatum in a manner that can be pharmacologically, functionally, and anatomically dissociated from dopamine release regulated by $\alpha 6 \beta 2^{*}$ and $(\alpha 6 \beta 2)(\alpha 4 \beta 2) *$ nAChRs (Salminen et al., 2004; Grady et al., 2010; Exley et al., 2012). For example, $(\alpha 4 \beta 2)_{2} \alpha 5$ nAChRs regulate the stimulatory effects of nicotine on dopamine release largely in dorsal striatum, whereas $\alpha 6^{*} \mathrm{nAChRs}$ regulate dopamine release in the NAc (Exley et al., 2012). In fact, $\alpha 5^{*}$ and $\alpha 6^{*}$ nAChRs on dopamine neurons are thought to be nonoverlapping populations that independently regulate striatal dopamine release (Exley et al., 2012). The functional significance of these different mechanisms of dopamine release is not yet clear but likely reflects dissociable dopaminerelated behaviors influenced by nicotine. The direct stimulatory effects of nicotine on VTA dopamine neurons are attenuated in $\alpha 5$ subunit knockout mice (Morel et al., 2014; Sciaccaluga et al., 2015) consistent with the attenuated striatal dopamine responses to nicotine in these mice. Virus-mediated re-expression of $\alpha 5$ subunits in VTA dopamine neurons of the $\alpha 5$ subunit knockout mice can "rescue" their responsiveness to nicotine (Morel et al., 2014; Sciaccaluga et al., 2015), further supporting an important role for $(\alpha 4 \beta 2)_{2} \alpha 5 \mathrm{nAChRs}$ in the dopamine-enhancing actions of nicotine. In contrast to $\alpha 4 \beta 2^{*}$ $\mathrm{nAChRs}$ in the midbrain (Akers et al., 2020), $(\alpha 4 \beta 2)_{2} \alpha 5$ nAChR's system is resistant to nicotine-induced upregulation (Mao et al., 2008), suggesting that adaptive changes in their expression levels do not contribute to the development of nicotine dependence.

\section{G. $\alpha 7 * n A C h R$ Subtypes and Nicotine Reward}

In contrast to the heteromeric nAChRs, behavioral evidence linking $\alpha 7$ homomeric nAChRs to the rewarding and reinforcing actions of nicotine is relatively weak. $\alpha 7 \mathrm{nAChRs}$ are thought to contribute to the stimulatory effects of nicotine on excitatory glutamatergic inputs to VTA dopamine neurons (Girod et al., 2000; 
Mansvelder and McGehee, 2000). Nevertheless, the rewarding effects of nicotine were unaltered in $\alpha 7$ nAChR subunit knockout mice compared with wild-type mice in a CPP procedure (Walters et al., 2006). Similarly, the acquisition of nicotine self-administration was unaltered relative to wild-type mice in an acute tailvein self-administration procedure (Pons et al., 2008). However, oral nicotine intake gradually extinguished over time in $\alpha 7$ knockout mice relative to wild-type mice using a two-bottle choice procedure (Levin et al., 2009). Female but not male $\alpha 7$ nAChR subunit knockout mice consumed less nicotine that their respective wild-type control groups in a two-bottle choice procedure (Bagdas et al., 2019). Intravenous nicotine self-administration was reduced by the putatively selective $\alpha 7 \mathrm{nAChR}$ antagonist methyllycaconitine (MLA) (Markou and Paterson, 2001). Complicating this finding is the fact that MLA can antagonize non- $\alpha 7$ nAChR subtypes (Bryant et al., 2002) and retains nicotine-related behavioral effects in $\alpha 7$ subunit knockout mice (Salas et al., 2007). Hence, caution should be exercised when attributing behavioral or physiologic effects of MLA to an action exclusively at $\alpha 7 \mathrm{nAChRs}$.

\section{H. Nicotine Modifies Impulse-Dependent and -Independent Accumbal Dopamine Release}

The studies exported above often employed in vivo microdialysis or ex vivo brain slice superfusion techniques to investigate the actions of nicotine on mesoaccumbens dopamine transmission (Di Chiara, 2000). Drawbacks of such approaches include the very long sampling times (order of minutes) over large portions of the striatum and often reflect nonsynaptic "spillover" of dopamine that escapes rapid reuptake or breakdown (Parsons and Justice, 1992; Zhou et al., 2001). More complex actions of nicotine on accumbal dopamine transmission have been revealed using more modern approaches that can capture rapid "synaptic" dopamine transmission in the accumbens. Using fast-scan cyclic voltammetry to monitor electrically evoked dopamine release, it was shown that nicotine inhibits action potential-dependent dopamine release in the NAc (Zhou et al., 2001). The nAChR antagonists mecamylamine and $\mathrm{DH} \beta \mathrm{E}$ mimicked this action of nicotine (Zhou et al., 2001). These data suggest that nicotine acted by desensitizing $\beta 2 * \mathrm{nAChRs}$ located on the terminals of dopamine neurons in the accumbens and hint at complex "multimodal" actions of nAChRs on accumbal dopamine transmission rather than the uniform nicotine-induced increases in dopamine release suggested by previous studies. The inhibitory effect of nicotine on dopamine release measured by voltammetry depends on the baseline activity of dopamine neurons. When dopamine release in the accumbens was stimulated using single electrical pulses to recapitulate tonic-like firing patterns (usually $2-5 \mathrm{~Hz}$ ), nicotine decreased dopamine release in a manner consistent with the desensitization of presynaptically located $\beta 2^{*} \mathrm{nAChRs}$ (Rice and Cragg, 2004; Zhang and Sulzer, 2004). However, when dopamine release was stimulated using multiple pulses in a manner that recapitulates burst-like firing patterns (usually $15-100 \mathrm{~Hz}$ ) thought to occur when rewarding or rewardpredictive stimuli are encountered (Schultz, 1986), nicotine instead increased dopamine release (Rice and Cragg, 2004; Zhang and Sulzer, 2004). Based on these findings, it was proposed that nicotine acts as a "highpass filter" that enhances the contrast between tonic and phasic patterns of dopamine neuron activity, with this action potentially contributing to the rewardenhancing properties of the drug (Rice and Cragg, 2004). Nicotine appeared to act in this manner by blocking short-term inhibitory plasticity in dopamine neurons (Rice and Cragg, 2004), which serves to limit dopamine release during periods of high activity (Cragg, 2003), and instead enhancing short-term calcium-dependent excitatory plasticity (Zhang and Sulzer, 2004).

The population of $\beta 2 * \mathrm{nAChRs}$ in the accumbens that is desensitized by nicotine to enhance activitydependent dopamine release was shown to contain $\alpha 4$, $\alpha 6$, and $\beta 3$ subunits (Exley and Cragg, 2008; Exley et al., 2011, 2012). This likely reflects the high-affinity $(\alpha 4 \beta 2)(\alpha 6 \beta 2) \beta 3$ nAChR subtype. By contrast, $\alpha 4$ (non$\alpha 6$ ), $\alpha 6$ (non- $\beta 3$ ), and $\alpha 6$ (non- $\alpha 4$ ) nAChRs play minimal roles in this action of nicotine (Exley et al., 2012). Interestingly, $\alpha 5$ subunit knockout mice did not show a desensitization-like enhancement of activity-dependent dopamine release in the accumbens, suggesting that they are not those nAChRs desensitized by nicotine to promote impulse-dependent dopamine release (Exley et al., 2012). However, the knockout mice showed greater sensitivity to the enhancing effects of the $\alpha 3 / \alpha 6^{*} \mathrm{nAChR}$ antagonist $\alpha$-conotoxin MII on activity-dependent dopamine release in the dorsal striatum (Exley et al., 2012), suggesting that $\alpha 5^{*}$ $n A C h R$ deficiency resulted in upregulation in the expression and function of $(\alpha 4 \beta 2)(\alpha 6 \beta 2) \beta 3 \mathrm{nAChRs}$ in dorsal striatum, likely to compensate for deficits in the function of $(\alpha 4 \beta 2)_{2} \alpha 5^{*} \mathrm{nAChRs}$ thought to specialize in regulating dopamine transmission in dorsal striatum (Champtiaux et al., 2002, 2003; Marubio et al., 2003; Salminen et al., 2004; Grady et al., 2010; Exley et al., 2011, 2012) (Fig. 2). The mechanisms by which desensitization of $(\alpha 4 \beta 2)(\alpha 6 \beta 2) \beta 3 \mathrm{nAChRs}$ can alleviate short-term inhibitory and promote shortterm excitatory plasticity in dopamine neurons to facilitate activity-dependent dopamine release are unclear but appear to involve dopamine D1 receptor signaling (Goutier et al., 2016). It is important to note that squirrel monkeys treated chronically with nicotine (3 weeks of drinking a nicotine-containing solution) showed attenuated accumbal dopamine release in response to low (tonic) or high (phasic) frequency 
electrical stimulation and had abolished sensitivity the effects of $\alpha$-conotoxin MII on dopamine release (Perez et al., 2012). This raises questions about the nature of nicotine-induced adaptive responses in $\alpha 6 \beta 2 * \mathrm{nAChR}$ function in the accumbens of human smokers and whether phasic dopamine release is increased or decreased by chronic nicotine exposure in smokers.

The fact that nAChRs located on dopamine terminals exert such a robust inhibitory influence on activity-dependent dopamine release suggests that endogenous acetylcholine derived from striatal cholinergic neurons controls the degree to which dopamine transmission is increased in the striatum when these cells fire in reward-relevant burst patterns of activity (Zhou et al., 2001; Brimblecombe et al., 2018). Indeed, optical stimulation of cholinergic interneurons in the striatum was shown to increase dopamine release in an impulse-independent manner through a direct action of acetylcholine at $\beta 2^{*} \mathrm{nAChRs}$ located on the terminals of dopamine neurons (Cachope et al., 2012; Threlfell et al., 2012). Hence, nAChR-mediated cholinergic transmission in the striatum is likely to facilitate dopamine release when midbrain dopamine neurons engage in tonic firing patterns but inhibit dopamine release when these cells engage in burst firing patterns (Cachope et al., 2012; Threlfell et al., 2012). By desensitizing nAChRs in the striatum, nicotine may facilitate reward-related burst firing in the accumbens to enhance brain reward function. Conversely, stimulation of $\mathrm{nAChRs}$ in accumbens may enhance dopamine release even when dopamine neurons are tonically firing, which may also contribute to reward-related behaviors (Cover et al., 2019). Precisely how these different sources of nAChR-related dopamine release interact and their relevance to discrete behavioral responses to nicotine are currently unclear.

\section{Dopamine Mechanisms of Nicotine Aversion}

In addition to its rewarding effects, nicotine also has aversive effects that motivate avoidance behaviors in humans, nonhuman primates, rats, and mice (Shoaib et al., 1997; Spealman, 1983; Sartor et al., 2010; Fowler et al., 2011). The same doses of nicotine that laboratory animals will work to obtain in self-administration experiments also have punishing properties that animals will work to avoid (Spealman, 1983). Whether nicotine is rewarding or aversive depends on whether the drug is consumed volitionally or delivered nonvolitionally, respectively (Spealman and Goldberg, 1978; Goldberg et al., 1981; Goldberg and Spealman, 1982; Spealman, 1983). Individuals who are less sensitive to the aversive effects of nicotine are more likely to be heavy smokers (Jensen et al., 2015). Similarly, those who suffer from psychiatric disorders associated with heavy smoking are often less sensitive to nicotine aversion (Williams et al., 2013), which likely contributes to their high levels of tobacco smoking. Sensiti-vity to nicotine aversion is thought to influence the likelihood of transitioning from occasional to regular tobacco use (Sartor et al., 2010; Fowler and Kenny, 2014). Interestingly, menthol and other additives contained in cigarettes and non-nicotine components of tobacco smoke can attenuate the aversive effects of nicotine (D'Silva et al., 2018; Harris et al., 2019). Hence, a better understanding of the neurobiological mechanisms of nicotine aversion may reveal new insights into genetic and environmental factors that influence vulnerability to tobacco dependence and the brain systems that undergo nicotine-induced adaptions to establish and maintain the tobacco smoking habit. As described above, the stimulatory effects of nicotine on midbrain dopamine neurons contribute to the rewarding properties of the drug that motivate tobacco smoking. However, dopamine transmission also regulates avoidance behaviors (Acquas et al., 1989; Shippenberg et al., 1993; Bromberg-Martin et al., 2010). This raises the possibility that midbrain dopamine neurons contribute to the aversive actions of nicotine. Consistent with this possibility, allelic variation in the genes that encode the dopamine D4 and D2 receptors has been shown to influence aversion-related responses to nicotine delivered by nasal spray in humans (Perkins et al., 2008). Lesioning the pedunculopontine tegmental nucleus (PPTg), which provides a major cholinergic projection to the VTA (Good and Lupica, 2009), blocked the rewarding effects of nicotine injected directly into the VTA and enhanced its aversive effects (Laviolette et al., 2002) (see Fig. 2). Infusion of a $\beta 2^{*}$ nAChR antagonist or a mixed $\alpha 7 / \alpha 6^{*} \mathrm{nAChR}$ antagonist into the VTA blocked the aversive effects of nicotine in a place conditioning procedure (Laviolette and van der Kooy, 2003b). These findings suggest that endogenous cholinergic transmission in the VTA derived from PPTg inputs and acting through locally expressed $\beta 2 *$ nAChRs regulates the rewarding and aversive properties of nicotine. Infusion of an NMDA glutamate receptor antagonist into the VTA similarly blocked the aversive effects of nicotine in a place conditioning procedure (Laviolette and van der Kooy, 2003b). Antagonism of NMDA receptors also rendered previously rewarding doses of nicotine aversive in rats, as measured using an ICSS procedure (Kenny et al., 2009). Nicotine-enhanced NMDA receptor-mediated glutamatergic transmission in the VTA contributes to the stimulatory effects of nicotine on accumbal dopamine release, with the role for NMDA receptor-mediated transmission in nicotine-enhanced dopamine release particularly important at higher doses of nicotine known to have aversive behavioral effects (Schilstrom 
et al., 1998; Fu et al., 2000). Hence, glutamatergic transmission in the VTA regulates components of dopamine transmission involved in both the rewarding and aversive effects of nicotine.

Focal lesion of dopamine inputs to the NAc shell accomplished by local infusion of the toxin 6-hydroxydopamine decreased the CPP response to intravenous nicotine injections in rats (Sellings et al., 2008). Conversely, lesion of dopamine inputs to the NAc core enhanced nicotine-induced CPP in rats (Sellings et al., 2008). NAc core dopamine lesions also attenuated conditioned taste avoidance triggered by intravenous nicotine infusions (Sellings et al., 2008). Systemic or intra-NAc injections of the mixed dopamine D1 and D2 receptor antagonist $\alpha$-flupenthixol blocked only the aversive effects of nicotine in a place conditioning procedure (Laviolette and van der Kooy, 2003a). Similarly, blockade of D2 dopamine receptors in the shell region of the NAc or D1 dopamine receptors in the NAc core abolished the aversive properties of nicotine injected directly into the VTA (Laviolette et al., 2008; Grieder et al., 2012). These findings provide compelling evidence that the rewarding and aversive effects of nicotine are encoded by VTAderived dopamine transmission in the accumbens, with the NAc core playing a prominent role in nicotine aversion. Notably, the aversive effects of nicotine were abolished in $\alpha 5 \mathrm{nAChR}$ subunit knockout mice in a manner that phenocopies the effects of dopamine receptor antagonists (Grieder et al., 2017). This suggests that the component of nicotine-enhanced dopamine transmission in the striatum mediated by $(\alpha 4 \beta 2)_{2} \alpha 5 \mathrm{nAChRs}$ may signal the aversive but not the rewarding effects of nicotine.

\section{Cellular Mechanisms in VTA of Nicotine Reward and Aversion}

\section{A. Balance between nAChR Signaling in VTA Dopaminergic and GABAergic Neurons}

Recent findings have shed important light on the cellular mechanisms in the VTA that regulate nicotine aversion. Using mice with floxed alleles of the Chrna4 gene, it was shown that Cre recombinasemediated conditional deletion of $\alpha 4 \mathrm{nAChR}$ subunits in the ventral midbrain increased nicotine self-administration (oral intake) only when high-concentration nicotine solutions were available (Peng et al., 2017). This pattern of nicotine self-administration behavior is thought to occur when the punishing properties of higher nicotine doses are attenuated, which disinhibits self-administration of aversive doses that would otherwise suppress intake (Fowler et al., 2011). $\alpha 4$ nAChR subunits were knocked down in both dopamine and GABAergic neurons in the VTA of mice in this study (Peng et al., 2017), raising the possibility that at least some fraction of those VTA dopamine neurons that express $\alpha 4^{*} \mathrm{nAChR}$ regulate the aversive reactions to nicotine. As noted above, virus-mediated re-expression of the Chrnb2 gene in VTA GABAergic neurons rescued nicotine-induced CPP in $\beta 2$ subunit knockout mice (Grieder et al., 2019). This study also reported that virus-mediated re-expression of Chrnb2 only in VTA dopamine neurons failed to rescue nicotine reward in $\beta 2$ subunit knockout mice but instead rendered the knockout mice sensitive to nicotine aversion (Grieder et al., 2019). Virus-mediated re-expression of Chrnb2 simultaneously in VTA dopamine and GABA neurons but not in either cell type alone was necessary to "rescue" nicotine-induced burst firing of VTA dopamine neurons and reconstitute sensitivity to the reinforcing properties of nicotine in $\beta 2$ subunit knockout mice, as measured using an intra-VTA self-administration procedure (Tolu et al., 2013). However, when Chrnb2 was re-expressed only in VTA GABA neurons in $\beta 2$ subunit knockout mice, these cells were persistently activated by nicotine, and only aversive behavioral responses to nicotine were detected (Tolu et al., 2013). This is consistent with the observation that VTA GABA neurons are more robustly activated by aversive than rewarding doses of nicotine (Dehkordi et al., 2018). These findings support a complex regulatory mechanism whereby concerted actions by nicotine on $\beta 2^{*}$ nAChRs expressed by both VTA dopamine and GABA neurons are required to trigger reward-relevant burst firing of dopamine neurons, with this action necessary to experience the reinforcing properties of nicotine that support self-administration behavior. By contrast, nicotine acting on VTA dopamine or GABA neurons alone can promote aversion (Tolu et al., 2013; Grieder et al., 2019), likely by blocking the ability of dopamine neurons to engage in burst firing.

\section{B. Anterior-Posterior Domains of the VTA}

The fact that VTA dopamine neurons regulate both the rewarding and aversive properties of nicotine reflects the remarkable functional heterogeneity of these cells, with discrete populations likely specializing in positive or negative reinforcement processes (Lammel et al., 2011, 2012, 2014). Dopamine neurons located in the posterior VTA (pVTA) but not the anterior VTA (aVTA) regulate the rewarding properties of nicotine that support self-administration behavior (Ikemoto et al., 2006) (Fig. 3). Similarly, ethanol, cocaine, opioids, and cholinergic agonists are all volitionally self-administered into the pVTA but are not reliably self-administered into the aVTA (Rodd-Henricks et al., 2000; Ikemoto and Wise, 2002; Zangen et al., 2002; David et al., 2004; Rodd et al., 2005). The pVTA but not aVTA also regulates the locomotor-stimulating properties of these drugs (Ikemoto et al., 2003; Sanchez-Catalan et al., 2009). aVTA and pVTA dopamine neurons are distinguished by distinct morphologic features (Zhao-Shea et al., 2011) and project 


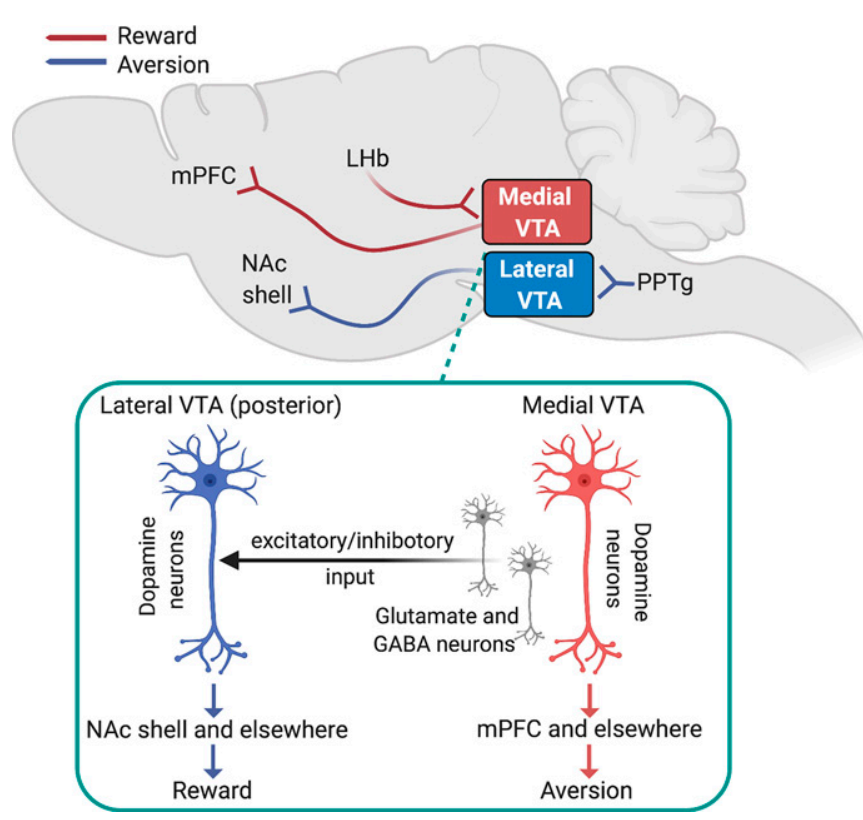

Fig. 3. Dopamine mechanisms of nicotine reward and aversion. Dopamine neurons located in the medial VTA that receive input from the LHb and project to the $\mathrm{mPFC}$ regulate aversion-related behaviors. Dopamine neurons located in the lateral VTA that receive input from the PPTg and project to the medial portion of the NAc shell regulate reward-related behaviors. Shown in the insert is the putative nAChR-regulated excitatory and inhibitory input from the medial VTA to the lateral VTA, particularly in posterior (caudal) VTA, that may regulate reward-related responses to nicotine.

to different regions of the striatum (Ikemoto et al., 2006). Specifically, pVTA dopamine neurons project predominantly to medial NAc shell and medial olfactory tubercle, whereas aVTA neurons instead project to the accumbens core, lateral tubercle, and dorsal striatum (Ikemoto et al., 2006). Dopamine neurons in the pVTA show greater responsiveness to nicotine than those in the aVTA, as measured by nicotineinduced cFos immunoreactivity (Zhao-Shea et al., 2011), consistent with the fact that pVTA contains denser concentrations of nAChRs than aVTA (ZhaoShea et al., 2011). Moreover, pVTA dopamine neurons are activated by rewarding doses of nicotine, whereas aVTA dopamine neurons are activated only by high concentrations of nicotine that have aversive properties (Fonck et al., 2005; ZhaoShea et al., 2011). Within the pVTA, $\alpha 4^{*}$ and $\alpha 6^{*}$ nAChR subtypes are thought to regulate the rewarding and reinforcing properties of nicotine (ZhaoShea et al., 2011; Exley et al., 2012; Liu et al., 2012; Engle et al., 2013).

\section{Medial-Lateral Domains of the VTA}

In addition to antero-posterior heterogeneity, VTA neurons also demonstrate a medial-lateral functional gradient (Lammel et al., 2011, 2012, 2014) (Fig. 3). Indeed, neurons in the medial VTA (VTA ${ }^{\mathrm{MED}}$ ) are more likely to be GABAergic or glutamatergic than neurons in the lateral VTA $\left(\mathrm{VTA}^{\mathrm{LAT}}\right)$, where the majority of the dopamine neurons are concentrated (Lammel et al., 2008; Hnasko et al., 2012; Root et al., 2014a,b; Ntamati and Luscher, 2016; Yan et al., 2018, 2019). Projections from the PPTg to the VTA are known to regulate the rewarding properties of nicotine (Corrigall et al., 2002; Alderson et al., 2006, 2008; Maskos, 2008). These PPTg inputs synapse preferentially onto neurons in lateral domains of the VTA (PPTg-VTA ${ }^{\mathrm{LAT}}$ neurons), which in turn project to the NAc medial shell (Lammel et al., 2012). Optical stimulation of PPTg-VTA ${ }^{\mathrm{LAT}}$ neurons can elicit reward-related behaviors that are blocked by dopamine receptor antagonists infused into the NAc shell (Lammel et al., 2012). Rewarding doses of nicotine are known to preferentially increase dopamine transmission in the NAc shell (Nisell et al., 1997; Lecca et al., 2006), which may reflect the recruitment of this PPTg-VTANAc shell reward circuit (Fig. 3). In addition to rewardrelevant $\mathrm{VTA}^{\mathrm{LAT}}$ dopamine neurons, cholinergic inputs from PPTg and the adjacent laterodorsal tegmental nucleus (LDTg) also project to $\mathrm{VTA}^{\mathrm{MED}}$ glutamatergic neurons that have been heavily implicated in aversionrelated behavioral states (Root et al., 2014a; Lammel et al., 2015; Qi et al., 2016; McGovern et al., 2020) and are known to express functional $\alpha 4^{*}$ and $\alpha 6^{*}$ nAChRs (Yan et al., 2018, 2019). Recent data have revealed that PPTg- and LDTg-derived cholinergic transmission in the VTA bidirectionally modulates reward and aversion behaviors (Dautan et al., 2016). This suggests that the rewarding and aversive effects of nicotine may reflect the nonphysiologic recruitment of these processes, which are normally controlled by endogenous cholinergic transmission acting at local nAChRs in the VTA (Fig. 3).

In contrast to PPTg inputs to the VTA, which predominately but nonexclusively target VTA ${ }^{\mathrm{LAT}}$ neurons, inputs from the lateral habenula synapse preferentially onto neurons in the VTA ${ }^{\mathrm{MED}}$ [lateral habenula ( $\left.\mathrm{LHb}\right)$ VTA $^{\text {MED }}$ neurons] (Fig. 3). VTA ${ }^{\text {MED }}$ neurons targeted by the $\mathrm{LHb}$ in turn project to the medial prefrontal cortex (mPFC) (Lammel et al., 2012) (Fig. 3). Optical stimulation of LHb-VTA ${ }^{\mathrm{MED}}$ neurons elicits aversion-related behaviors that are blocked by dopamine receptor antagonists infused in the mPFC (Lammel et al., 2012). Nicotine is known to increase dopamine release in the mPFC (Rossi et al., 2005), which could reflect the engagement of this LHb-VTA-mPFC aversion circuit. VTA ${ }^{\mathrm{MED}}$ dopamine neurons send a reciprocal projection back to the LHb, with these VTA ${ }^{\mathrm{MED}}$ dopamine neurons known to corelease both glutamate and GABA (Root et al., 2014b) (Fig. 3). VTA-derived glutamate transmission in the $\mathrm{LHb}$ regulates aversion-related behaviors (Root et al., 2014a), whereas VTA-derived GABA transmission in the $\mathrm{LHb}$ regulates rewardrelated behaviors (Stamatakis et al., 2013). Notably, nicotine increases both glutamatergic and GABAergic transmission in the LHb, likely by stimulating $\alpha 4 \beta 2 *$ 
and $\alpha 6^{*}$ nAChRs expressed by VTA dopamine inputs to the habenula (Zuo et al., 2016). This suggests that nicotine bidirectionally modulates the activity of aversion-relevant neurons in the LHb by altering the balance of VTA-derived glutamatergic and GABAergic transmission. In addition to their projections to aversion-related neurons in $\mathrm{VTA}^{\mathrm{MED}}$, LHb neurons also send a prominent projection to GABA neurons in the rostromedial tegmental nucleus (RMTg) (Jhou et al., $2009 \mathrm{~b}$ ), also known as the "tail" of the VTA. In turn, RMTg GABA neurons project to the VTA where they can inhibit dopamine neurons to elicit aversion-related behaviors (Jhou et al., 2009a,b; Hong et al., 2011; Barrot et al., 2012). Nicotine markedly enhances the activity of RMTg neurons (Lecca et al., 2011), providing another mechanism by which nicotine can modulate the activity of reward-related dopamine neurons in the VTA. Hence, nAChR-induced modulation of synaptic inputs to VTA ${ }^{\mathrm{LAT}}$ and VTA ${ }^{\mathrm{MED}}$ neurons and modulation of the projections from VTA ${ }^{\mathrm{LAT}}$ and VTA ${ }^{\mathrm{MED}}$ neurons to downstream brain sites likely contribute to the reinforcing actions of nicotine (Fig. 3).

Recent studies have begun to reveal the complex interplay between dopamine neurons located in medial and lateral domains of the VTA and how nicotine modifies these interactions (Fig. 3). Specifically, VTA ${ }^{\mathrm{MED}}$ neurons were shown to provide both excitatory and inhibitory input to VTA ${ }^{\mathrm{LAT}}$ dopamine neurons (Yan et al., 2019). Interestingly, a discrete subpopulation of the VTA ${ }^{\mathrm{MED}}$ neurons expresses $\beta 2^{*}$ nAChRs and coreleases both glutamate and GABA onto VTA ${ }^{\mathrm{LAT}}$ dopamine neurons (Yan et al., 2019). Nicotine had bidirectional effects on $\mathrm{VTA}^{\mathrm{MED}}$-derived excitatory glutamatergic transmission in VTA ${ }^{\mathrm{LAT}}$ dopamine neurons, increasing excitatory input to approximately half of the recorded neurons in VTA ${ }^{\mathrm{LAT}}$ and decreasing excitatory input to the other half (Yan et al., 2019). By contrast, nicotine uniformly decreased $\mathrm{VTA}^{\mathrm{MED}}$-derived inhibitory transmission in $\mathrm{VTA}^{\mathrm{LAT}}$ dopamine neurons (Yan et al., 2019). These findings suggest that nicotine-induced inhibition of $\mathrm{VTA}^{\mathrm{MED}}$-derived GABAergic input and simultaneous stimulation of $\mathrm{VTA}^{\mathrm{MED}}$-derived glutamatergic input to VTA ${ }^{\mathrm{LAT}}$ "reward" dopamine neurons contribute to the rewarding properties of the drug, whereas nicotine-induced inhibition of $\mathrm{VTA}^{\mathrm{MED}}$-derived glutamatergic input to $\mathrm{VTA}^{\mathrm{LAT}}$ reward neurons contributes to the aversive properties of the drug. More broadly, these findings suggest that complex modulation of excitatory and inhibitory transmission onto VTA ${ }^{\mathrm{MED}}$ and VTA ${ }^{\mathrm{LAT}}$ dopamine neurons by nicotine acting through local neurons within the VTA and on long-range inputs to the VTA determines whether nicotine has rewarding or aversive effects. Further investigation will be required to better define the complex effects of nicotine on local and long-range synaptic inputs to $\mathrm{VTA}^{\mathrm{MED}}$ and VTA ${ }^{\mathrm{LAT}}$ dopamine neurons and the consequences of these actions on downstream brain sites.
The findings described above suggest that VTAderived dopamine, glutamate, and GABA transmission in the accumbens, $\mathrm{LHb}, \mathrm{mPFC}$, and elsewhere in the brain likely contribute to aversion-related responses to nicotine. However, dopamine receptor antagonists block nicotine aversion only in animals with a limited history of nicotine exposure (Tan et al., 2009). By contrast, the same dopamine manipulations block the rewarding effects of nicotine in animals that have previously been exposed to the drug (Tan et al., 2009). This apparent switch in the role for dopamine transmission that occurs with repeated exposure to nicotine from initially regulating nicotine aversion to instead regulating nicotine reward coincides with an increase in the incidence of nicotine-induced burst firing of VTA dopamine neurons and a decrease in the baseline activity of VTA GABAergic neurons (Tan et al., 2009). Hence, VTA dopamine neurons may participate in aversive reactions to nicotine that influence the likelihood of transitioning from initial tobacco use to regular intake (de Wit \& Phillips 2012). Furthermore, adaptive responses in dopamine-mediated aversion systems driven in part by alterations in the function of VTA GABA neurons may contribute to this transition process. However, these findings also suggest that dopamine transmission is unlikely to participate in aversive responses to nicotine once regular nicotine use has been established and that other aversion-related brain systems influence patterns and amounts of tobacco smoking in smokers (Fowler and Kenny, 2014). This raises the important issue of the identity of dopamine-independent brain systems that regulate nicotine aversion in tobacco smokers and nicotine-experienced laboratory animals, which is considered in more detail below.

\section{Nondopamine Mechanisms of Nicotine Aversion}

\section{A. Human Genetics Reveal nAChR Subtypes that Regulate Nicotine Intake}

As noted above, nicotine has rewarding properties that motivate intake and aversive properties that motivate avoidance. These competing positive and negative effects likely explain the inverted U-shape of the dose-response curve for self-administered nicotine seen in humans, nonhuman primates, and laboratory rodents responding under fixed-ratio schedules. Lower doses elicit primarily rewarding effects that motivate self-administration, whereas higher doses elicit mixed rewarding/aversive effects that necessitate careful titration of intake (Goldberg and Spealman, 1982; Risner and Goldberg, 1983; Corrigall and Coen, 1989; Harvey et al., 2004; DeNoble and Mele, 2006; Fowler and Kenny, 2011). One potential explanation for these opposing motivational properties of nicotine and the shape of the dose-response curve is that the same population of nAChRs regulates both the 
rewarding and aversive effects of nicotine, with lower doses activating these nAChRs and higher doses desensitizing and thereby inactivating these nAChRs (Pidoplichko et al., 1997). An alternative explanation is that lower unit doses of nicotine engage high-affinity nAChRs located in brain reward circuits to motivate nicotine selfadministration, whereas higher nicotine doses engage low-affinity nAChRs in brain aversion circuits that motivate nicotine avoidance. According to this framework, nicotine intake is titrated to maximize the activation of reward-related nAChRs while minimizing the activation of aversion-related nAChRs. The mesoaccumbens dopamine system is enriched in $(\alpha 4 \beta 2)(\alpha 6 \beta 2) \beta 3$ nAChRs (Grady et al., 2007), which have the highest sensitivity to nicotine of any native nAChR so far identified (Grady et al., 2007) and are thought to contribute to nicotine reward. Until recently, little was known about the identity of the putative "low-affinity" nAChRs activated by higher nicotine doses or the contributions of the brain regions in which they are located to the control of nicotine intake.

The CHRNA5-CHRNA3-CHRNB4 gene cluster located in chromosome region $15 \mathrm{q} 25$ encodes the $\alpha 5, \alpha 3$, and $\beta 4$ $\mathrm{nAChR}$ subunits, respectively. nAChRs that incorporate subunits encoded by this gene cluster are known as "low-affinity" nAChRs because they bind nicotine far less efficiently than the so-called high affinity $\beta 2^{*}$ nAChRs (Zoli et al., 1998). They are also known as the ganglionic $\mathrm{nAChRs}$ because of their dense expression in neurons of the autonomic nervous system (Kemp and Morley, 1986). Large-scale human genetics studies have shown that allelic variation across the entire CHRNA5CHRNA3-CHRNB4 gene cluster is associated with increased vulnerability to tobacco dependence and higher numbers of cigarettes smoked per day (Amos et al., 2008; Ware et al., 2011; Marques-Vidal et al., 2011; Gallego et al., 2013; Liu et al., 2018; Perez-Morales et al., 2018). In particular, individuals who carry risk alleles in CHRNA5 are less sensitive to the aversive effects of nicotine (Jensen et al., 2015) and more likely to be heavy smokers (Saccone et al., 2007; Berrettini et al., 2008; Lips et al., 2009; Ohi et al., 2019). A particular variant in CHRNA5 (rs16969968) that gives rise to an amino-acid substitution $(\alpha 5-398 \mathrm{D} \rightarrow \alpha 5-398 \mathrm{~N})$ thought to decrease the function of $\alpha 5^{*} \mathrm{nAChRs}$ that incorporate the mutant subunit increases tobacco dependence risk by $\sim 30 \%$ in individuals who carry a single copy of the gene variant and doubles the risk in individuals who carry two copies of the variant (Bierut et al., 2008; Wang et al., 2009). Alleles in CHRNA5 are also associated with heavy smoking (Berrettini et al., 2008; Bierut et al., 2008; Grucza et al., 2008; Stevens et al., 2008), early onset of smoking behavior (Weiss et al., 2008), and with "pleasurable buzz" from tobacco (Sherva et al., 2008). Hence, low-affinity $\alpha 3 \beta 4^{*} \mathrm{nAChRs}$ and $\alpha 5^{*} \mathrm{nAChR}$ subtypes are of considerable interest in the context of nicotine aversion.

\section{B. $\alpha 5^{*}, \alpha 3^{*}$, and $\beta 4^{*} n A C h R$ R Regulate Nicotine} Avoidance

As noted above, the so-called "ganglionic" $\alpha 3 \beta 4^{*}$ nAChRs are expressed in the CNS, where they account for low-affinity nicotine binding sites (Flores et al., 1992). They are far less abundant than $\alpha 4 \beta 2 *$ nAChRs and are concentrated in just a few brain regions, most notably the medial habenula and interpeduncular nucleus (Clarke et al., 1985; Cimino et al., 1992). In heterologous expression systems, $\alpha 5$ subunits can incorporate into the $\alpha 3 \beta 4^{*}$ nAChRs, which modifies their sensitivity to agonist-induced desensitization (Wang et al., 1996; Mao et al., 2008; Gotti et al., 2007; Perry et al., 2007; Kuryatov et al., 2008). However, $\alpha 5$ subunits incorporate more efficiently into $\alpha 4 \beta 2 \mathrm{nAChRs}$ (Gotti et al., 2007; Perry et al., 2007; Kuryatov et al., 2008; Mao et al., 2008). Similar to $\alpha 5, \beta 3$ can also functions as an accessory subunit in $\alpha 3 \beta 4^{*}$ and $\alpha 3 \beta 2^{*}$ nAChRs (Gotti et al., 2009). $\beta 3$ has a dominant-negative function when incorporated into $\alpha 3 \beta 4^{*} \mathrm{nAChRs}$, rendering the resulting receptor complex nonfunctional (Palma et al., 1999; Broadbent et al., 2006). Less frequently, $\alpha 3 \mathrm{nAChRs}$ can coassemble with $\beta 2$ subunits to form a functional $\alpha 3 \beta 2 *$ nAChR subtype.

$\alpha 5$ nAChR subunit knockout mice and their wildtype littermates intravenously self-administer nicotine according to an inverted U-shaped dose-response curve (Fowler et al., 2011), as expected based on previous findings in humans and laboratory animals (Goldberg et al., 1981; Henningfield and Goldberg, 1983; Corrigall and Coen, 1989; Le Foll et al., 2007). Levels of nicotine self-administration were similar in $\alpha 5$ subunit knockout and wild-type mice on the "ascending" portion of the dose-response curve when lower unit doses of nicotine were available (Fowler et al., 2011). By contrast, responding was much higher in the knockout mice than their wild-type littermates when higher unit doses of nicotine were on the "descending" portion of the dose-response curve (Fowler et al., 2011). Transgenic rats that express a mutant form of the $\alpha 5 \mathrm{nAChR}$ subunit gene modified to contain the same amino-acid substitution caused by the rs16969968 risk allele also self-administered greater quantities of nicotine than wild-type rats but only when higher unit doses of nicotine on the descending portion of the dose-response curve were available (Forget et al., 2018). Enhanced responding for nicotine as the unit dose increases is thought to reflect an intensification of the reinforcing properties of the drug, thereby motivating higher levels of intake (Lynch and Carroll, 2001). Diminished responding for nicotine as the dose increases reflects greater restraint over intake to avoid the increasingly aversive effects of higher drug doses (Henningfield and Goldberg, 1983; Lynch and Carroll, 2001), more rapid 
development of drug satiation (Lynch and Carroll, 1999, 2001), or the manifestation of performance-disrupting actions of the drug (Lynch and Carroll, 1999, 2001). Hence, deletion of $\alpha 5 \mathrm{nAChR}$ subunits has dissociable effects on the motivational processes that control nicotine intake. The stimulatory effects of nicotine on brain reward systems that motivate nicotine use were unaltered by $\alpha 5$ subunit knockout, whereas the aversive effects of nicotine that limit its self-administration were attenuated by $\alpha 5^{*}$ nAChR deficiency. Consistent with this interpretation, $\alpha 5 \mathrm{nAChR}$ subunit knockout mice had similar sensitivity to the rewarding effects of nicotine as wild-type mice but markedly reduced sensitivity to the aversive effects of nicotine, as measured using CPP and ICSS procedures (Jackson et al., 2010; Fowler et al., 2011, 2013; Grieder et al., 2017). More recently, Chrna $3^{\text {tm1.1Hwrt }}$ hypomorphic mice, which express much lower levels of $\alpha 3 \mathrm{nAChR}$ subunits than wild-type mice (Caffery et al., 2009), were shown to self-administer greater numbers of intravenous nicotine infusions than their wild-type littermates, with this effect most apparent when nicotine doses on the descending portion of the dose-response curve were available (Elayouby et al., 2021). Similarly, $\beta 4 \mathrm{nAChR}$ subunit knockout mice also self-administered greater numbers of nicotine infusions directly into the VTA when higher nicotine doses were available (Husson et al., 2020), although these animals self-administered less nicotine across all doses tested when the drug was delivered by intravenous infusion (Harrington et al., 2016). Transgenic mice that overexpressed $\beta 4 \mathrm{nAChR}$ subunits in only those neurons that constitutively express $\beta 4$ subunits showed enhanced nicotine aversion and decreased oral nicotine intake (Frahm et al., 2011). Similarly, lentivirus-mediated expression in the brains of mice of mutant gain-of-function $\beta 4 \mathrm{nAChR}$ subunits, which incorporated $C H R N B 4$ variants associated with reduced risk of tobacco dependence, increased aversion to nicotine (Slimak et al., 2014). Together, these findings suggest that $\alpha 5^{*}, \alpha 3^{*}$, and $\beta 4^{*}$ nAChRs regulate nicotine aversion.

\section{Low-Affinity nAChRs Are Enriched in Medial Habenula and Interpeduncular Nucleus}

$\alpha 3$ and $\beta 4 \mathrm{nAChR}$ subunit transcripts are expressed at low levels in the midbrain dopamine system (Klink et al., 2001; Azam et al., 2002), and functional $\alpha 3^{*}$ and $\beta 4^{*} \mathrm{nAChRs}$ have not been reliably detected in the VTA. $\alpha 5$ subunit transcripts are expressed at modest levels in the VTA, although functional $(\alpha 4 \beta 2)_{2} \alpha 5 \mathrm{nAChRs}$ are thought to be expressed by dopamine and nondopamine neurons in the VTA (Fig. 2). By contrast, high densities of $\alpha 5, \alpha 3$ and $\alpha 4 \mathrm{nAChR}$ subunit transcripts are detected in the $\mathrm{mHb}$, interpeduncular nucleus (IPn), and nucleus of the solitary tract (nTS) (Marks et al., 1992; WinzerSerhan and Leslie, 1997; Sheffield et al., 2000; De Biasi and Salas, 2008; O'Leary et al., 2008; Grady et al., 2009;
Frahm et al., 2011; Tuesta et al., 2011, 2017; Hsu et al., 2013; Antolin-Fontes et al., 2015; Morton et al., 2018; Husson et al., 2020). The $\mathrm{mHb}$ is located immediately adjacent to the LHb in the epithalamus, projects almost exclusively to the IPn, and is subdivided into four functional domains: superior, inferior, central, and lateral nuclei (Aizawa et al., 2012). Neurons in the inferior, central, and lateral domains produce acetylcholine, whereas those in the superior portion produce substance $\mathrm{P}$ (neurokinin 1) or the cytokine interleukin-18 (Contestabile et al., 1987; Andres et al., 1999; Aizawa et al., 2012; Kobayashi et al., 2013; Quina et al., 2017). All $\mathrm{mHb}$ neurons are thought to produce and corelease glutamate, which is considered the major functional transmitter at the habenula-IPn synapse (Ren et al., 2011; Aizawa et al., 2012; Aizawa and Zhu, 2019). The cholinergic neurons in ventral portions of the $\mathrm{mHb}$ project to central, rostral, and intermediate domains of the IPn (Kimura et al., 1981; Houser et al., 1983; Qin and Luo, 2009) where they assume highly unusual "serpentine" patterns of innervation (Ramón y Cajal, 1953; Lenn, 1976; Herkenham and Nauta, 1979; Lenn et al., 1983), whereas substance $\mathrm{P}$-positive neurons in dorsal $\mathrm{MHb}$ neurons project to the lateral domains of the IPn (Qin and Luo, 2009). nAChRs located on the presynaptic terminals of $\mathrm{mHb}$ inputs to the IPn regulate neurotransmitter release into the IPn, and the mHb-IPn synapse has long served as a model system for nAChR signaling in the brain (Sastry, 1978; Brown et al., 1983; McGehee et al., 1995). In addition, postsynaptic nAChRs located on IPn neurons can generate retrograde signaling molecules, including endocannabinoids and nitric oxide, that provide a source of feedback inhibition onto $\mathrm{mHb}$ inputs (Rodrigo et al., 1994; Ables et al., 2017; Quina et al., 2017; Melani et al., 2019). Little is currently known about the function of the $\mathrm{mHb}-\mathrm{IPn}$ circuit, although available data suggest that $\mathrm{mHb}$-derived cholinergic, glutamatergic, and substance $\mathrm{P}$ transmission in the IPn modulated by $\mathrm{GABA}_{\mathrm{B}}$, cannabinoid 1 (CB1), or glycine receptors regulates the encoding of memories related to aversive stimuli or events (Thompson, 1960; Donovick et al., 1970; Agetsuma et al., 2010; Yamaguchi et al., 2013; Soria-Gomez et al., 2015; Zhang et al., 2016; Koppensteiner et al., 2017; Geng et al., 2019; Melani et al., 2019). The mHb-IPn circuit may also coordinate adaptive physiologic and behavioral responses to stressful or anxiety-provoking stimuli (Lisoprawski et al., 1981; Murray et al., 1994; Shumake et al., 2003; Hsu et al., 2012, 2016; Xu et al., 2018; Duncan et al., 2019; Sherafat et al., 2020).

\section{D. nAChR Signaling in the mHb-IPn Circuit Regulates Nicotine Avoidance}

Considering the high densities of $\alpha 5, \alpha 3$ and $\alpha 4$ nAChR subunits in the habenula-IPn circuit (Marks et al., 1992; De Biasi and Salas, 2008) and the role for this circuit in regulating responses to aversive 
stimuli, it is perhaps not surprising that evidence has accumulated over the past decade implicating the mHb-IPn circuit in coordinating aversive behavioral responses to nicotine. Infusion of the local anesthetic lidocaine into the $\mathrm{mHb}$ or IPn of rats increased their nicotine intake via intravenous infusions (Fowler et al., 2011), suggesting that the mHb-IPn circuit exerts an inhibitory influence over nicotine-taking behavior. IPn neurons that receive excitatory inputs from the $\mathrm{mHb}$ are perhaps the most nicotine-responsive cells in the brain, as measured by nicotineinduced cFos immunoreactivity (Ren and Sagar, 1992; Pang et al., 1993; Salminen et al., 2000). Nicotineinduced cFos expression in IPn was greatly reduced in $\alpha 5$ subunit knockout mice compared with wild-type mice (Fowler et al., 2011). nAChR function was also decreased in the $\mathrm{mHb}$ and IPn of $\alpha 5$ subunit knockout mice, as measured by the rubidium efflux assay (Fowler et al., 2011). By contrast, nicotine-induced activation of the VTA was similar in wild-type and $\alpha 5$ knockout mice (Fowler et al., 2011). This suggests that $\alpha 5^{*}$ nAChRs are an important functional subtype that regulates the stimulatory effects of nicotine on the mHb-IPn circuit. The elevated level of nicotine self-administration in $\alpha 5$ subunit knockout mice was normalized by virus-mediated re-expression of $\alpha 5$ subunits specifically in the mHb-IPN circuit (Fowler et al., 2011). Conversely, lentivirus-mediated expression of short interfering RNAs (shRNAs) to knock down $\alpha 5$ or $\alpha 3$ nAChR subunits in the mHb or IPn increased nicotine self-administration in rats (Fowler et al., 2011; Elayouby et al., 2021), with these effects most apparent when higher unit doses were available. Conversely, lentivirus-mediated expression of mutant gain-of-function $\beta 4 \mathrm{nAChR}$ subunits in the $\mathrm{mHb}$ of mice increased nicotine aversion and decreased their nicotine intake (Slimak et al., 2014). Infusion of the $\alpha 3 \beta 4^{*}$ nAChR antagonists 18-methoxycoronaridine (18-MC) or $\alpha$-conotoxin AuIB into the $\mathrm{mHb}$ or IPn but not the $\alpha 3 \beta 2^{*}$ nAChR antagonist $\alpha$-conotoxin MII increased nicotine intake in rats (Fowler et al., 2011; Elayouby et al., 2021). By contrast, infusion of 18-MC into the VTA had no effect on nicotine self-administration in rats (Glick et al., 2011). These findings suggest that stimulatory effects of nicotine on the $\mathrm{mHb}$ IPn circuit mediated by $\alpha 5^{*}$ and $\alpha 3 \beta 4^{*} \mathrm{nAChRs}$ decrease the motivation to consume nicotine.

\section{E. nAChR Signaling in mHb-IPn Circuit Regulates the Reward-Inhibiting Effects of Nicotine}

Using an ICSS procedure, it was shown that shRNA-mediated knockdown of $\alpha 5 \mathrm{nAChR}$ transcripts in the mHb-IPn circuit of rats had no effects on the threshold-lowering effects of lower nicotine doses but attenuated the threshold-elevating effects of higher nicotine doses in rats (Fowler et al., 2011). These findings in rats recapitulate the same pattern of effects described above in $\alpha 5$ nAChR subunit knockout mice (Fowler et al., 2013). The ICSS threshold-lowering doses of nicotine are thought to reflect nicotineinduced enhancement of brain reward activity (Kenny and Markou, 2006). Conversely, the ICSS thresholdelevating effects of nicotine are through to reflect the reward-inhibiting effects of the drug that contribute to nicotine aversion and motivate nicotine avoidance (Schaefer and Michael, 1986; Fowler et al., 2011). Rats regulate their intravenous nicotine self-administration to achieve maximal lowering of ICSS thresholds (Kenny and Markou, 2006), suggesting that nicotine intake is titrated to obtain this action of nicotine while avoiding the threshold-elevating effects of the drug. Hence, disruption of $\alpha 5^{*}$ nAChR-mediated signaling in the $\mathrm{mHb}-\mathrm{IPn}$ circuit likely increases nicotine intake by attenuating the reward-inhibiting effects of nicotine and extending the range of nicotine doses that are reward-enhancing (Fowler et al., 2011). This is consistent with the observation that aversive responses to nicotine are attenuated in individuals who carry the rs16969968 polymorphism in CHRNA5 (Jensen et al., 2015). In contrast to dopamine-mediated mechanisms of nicotine aversion, which were detected only when animals had limited previous exposure to nicotine (Tan et al., 2009) (see above), the aversion mediated by the mHb-IPn circuit was observed even in animals that had experienced extended periods of daily access to high unit doses of nicotine (Fowler et al., 2011; Elayouby et al., 2021). This suggests that nAChR transmission in the mHbIPn circuit is likely to control nicotine intake in human smokers even after the habit has been established. Indeed, functional brain imaging studies have shown that nicotine modulates habenular activity in both nonsmokers and habitual smokers (Flannery et al., 2019; Jennings et al., 2020). Notably, mHb neurons receive excitatory and inhibitory synaptic input almost exclusively from the posterior septum via the stria medullaris (Sperlagh et al., 1998; Qin and Luo, 2009; Yamaguchi et al., 2013; Otsu et al., 2018), with ATP thought to function as a neurotransmitter at the septo-mHb synapse (Edwards et al., 1992; Sperlagh et al., 1995). Nevertheless, little is known about function of these septal inputs to the $\mathrm{mHb}$, and their potential involvement in behavioral responses to nicotine has not been explored.

\section{F. Stoichiometries of nAChRs Expressed in the mHb-IPn Circuit}

The mHb contains some of the highest concentrations of nAChRs in the brain, particularly those that account for the "low-affinity" nicotine binding sites (i.e., do not contain $\beta 2$ subunits) (Clarke et al., 1985; Wada et al., 1989; Marks et al., 1992; Zoli et al., 1998). As noted above, the highest concentrations of $\alpha 5, \alpha 3$, and $\alpha 4$ nAChR subunits in the brain are 
detected in the $\mathrm{mHb}$ and IPn. nAChRs expressed by $\mathrm{mHb}$ neurons are highly calcium-permeable (Mulle et al., 1992; Guo and Lester, 2007) and are expressed along the entire length of $\mathrm{mHb}$ neurons, including their soma, axons, and terminals in the IPn (Mulle et al., 1991; Lena et al., 1993; Passlick et al., 2018). Electrophysiological recordings of $\mathrm{mHb}$ neurons revealed two discrete $\mathrm{nAChR}$-mediated currents, suggesting that at least two populations of $n A C h R s$ are expressed in the mHb (Mulle et al., 1991; Connolly et al., 1995). Both nAChR populations are desensitized by nicotine (Lester and Dani, 1995; Hicks et al., 2000; Duncan et al., 2019). Neurons in the ventrolateral portion of the $\mathrm{mHb}$ are most responsive to nicotine (Fonck et al., 2009; Shih et al., 2014), with $\alpha 3$ and $\beta 4 \mathrm{nAChR}$ subunits expressed by most neurons in this region (Shih et al., 2014). Nicotine-responsive neurons in ventrolateral $\mathrm{mHb}$ provide synaptic inputs to rostral and dorsomedial domains of the IPn (Mulle et al., 1991; Shih et al., 2014). Blockade of $\alpha 3 \beta 4^{*}$ nAChRs has been shown to greatly reduce but not eliminate the stimulatory effects of nicotine on $\mathrm{mHb}$ neurons (Quick et al., 1999; Elayouby et al., 2021). Immunoprecipitation studies support these findings and suggest that relatively uncommon nAChR subtypes are expressed by $\mathrm{mHb}$ neurons (Fig. 4). Specifically, $\mathrm{mHb}$ neurons express high concentrations of $\alpha 3 \beta 4^{*} \mathrm{nAChRs}$ but also $\alpha 3 \beta 2^{*}, \alpha 3 \alpha 4 \beta 4^{*}$, and $\alpha 4 \beta 2^{*}$; nAChRs (Grady et al., 2009); and much smaller populations of $\alpha 2 \beta 2^{*}$ and $\alpha 6 \beta 2^{*}$ nAChRs (Fig. 4). A large proportion of these nAChRs contained $\alpha 5$ accessory subunits, which associate primarily with $\beta 2 * \mathrm{nAChR}$ stoichiometries (Grady et al., 2009). A large proportion of the $\mathrm{nAChRs}$ in the $\mathrm{mHb}$ also contained $\beta 3$ accessory subunits, which were most often found in combination with $\beta 4^{*}$ nAChRs (Grady et al., 2009). This contrasts with ventral midbrain dopamine neurons, in which $\beta 3$ subunits are exclusively almost expressed within $\alpha 6^{*}$ nAChRs. In the IPn, $\alpha 2 \beta 2^{*}$, $\alpha 3 \beta 2^{*}, \alpha 4 \beta 2^{*}, \alpha 3 \beta 4^{*}, \alpha 4 \beta 4^{*}$, and $\alpha 6 \beta 4^{*}$ nAChRs have been detected (Grady et al., 2009). Again, a large proportion of these contained $\alpha 5$ or $\beta 3$ accessory subunits, with $\alpha 5$ subunits associating primarily with $\beta 2 *$ $\mathrm{nAChRs}$ and $\beta 3$ with $\beta 4^{*} \mathrm{nAChRs}$ (Grady et al., 2009) (Fig. 4).

As noted above, a large proportion of nAChRs in the $\mathrm{mHb}$ contain $\alpha 5$ accessory subunits (Grady et al., 2009) and $\mathrm{nAChR}$ function in the $\mathrm{mHb}$ was markedly reduced in $\alpha 5$ subunit knockout mice when measured using rubidium efflux from habenular synaptosomes (Fowler et al., 2011). Habenular synaptosomes generated from $\beta 2$ but not $\beta 4$ knockout mice also showed striking deficits in nAChR function (Grady et al., 2009). These findings suggest that $\alpha 5^{*} \mathrm{nAChRs}$ play an important role in the $\mathrm{mHb}$ (Fig. 4). However, $\alpha 5$ subunit mRNA transcripts are expressed at very low levels by $\mathrm{mHb}$ neurons,

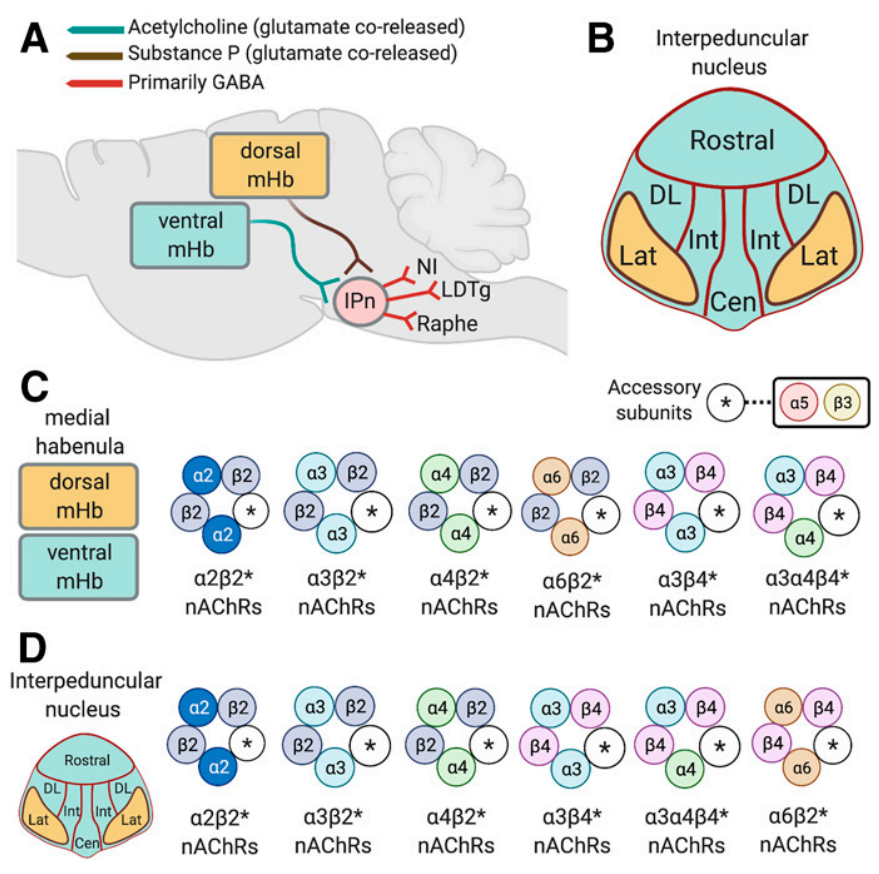

Fig. 4. Organization and $\mathrm{nAChR}$ subtypes of the habenula-interpeduncular nucleus circuit. (A) Substance P-expressing neurons in in the dorsal region of the $\mathrm{mHb}$ and cholinergic neurons in the ventral $\mathrm{mHb}$ corelease glutamate and project to the IPn. The IPn send predominately GABAergic projections to the raphe nuclei, LDTg, and the nucleus incertus (NI). (B) Graphical representation of subregions of the interpeduncular nucleus, including the rostral, dorsolateral (DL), intermediate (I), and central (Cen) nuclei that receive input from cholinergic neurons in the ventral $\mathrm{mHb}$. (C) Major stoichiometries of nAChRs predicted to be expressed presynaptically and postsynaptically in the $\mathrm{mHb}$. (D) Major stoichiometries of nAChRs predicted to be expressed presynaptically and postsynaptically in the $\mathrm{mHb}$ in the IPn.

with far higher concentrations found in the IPn (Sheffield et al., 2000; Hsu et al., 2013; Morton et al., 2018), although $\alpha 5 \mathrm{nAChR}$ subunit protein is detected in the $\mathrm{mHb}$ (Grady et al., 2009). As nAChR signaling in synaptosomes primarily reflects presynaptic nAChRs, these data suggest that $\alpha 4 \beta 2 \alpha 5^{*}$ nAChRs are the major functional $\mathrm{nAChR}$ subtype expressed on the terminals of synaptic inputs to $\mathrm{mHb}$ neurons. Consistent with this notion, nicotine increases the intrinsic excitability of $\mathrm{mHb}$ neurons through an action that depends on $\alpha 5^{*}$ $\mathrm{nAChRs}$ contained on presynaptic terminals in the $\mathrm{mHb}$ (Dao et al., 2014), with these presynaptic $\alpha 5^{*}$ nAChRs regulating the release of neurokinins into the $\mathrm{mHb}$, which act at postsynaptic neurokinin receptors located on $\mathrm{mHb}$ neurons (Dao et al., 2014).

This raises the important question of which nAChR subtypes into which $\alpha 5$ subunits assemble in the $\mathrm{mHb}$ IPn circuit to regulate behavioral responses to nicotine. In heterologous expression systems, $\alpha 5 \mathrm{nAChR}$ subunits can coassemble into $\alpha 4 \beta 2, \alpha 3 \beta 2$, and $\alpha 3 \beta 4$ nAChR subtypes (Fucile et al., 1997; Gerzanich et al., 1998; Tapia et al., 2007). However, in the brain $\alpha 5$ subunits preferentially assemble into $\alpha 4 \beta 2^{*} \mathrm{nAChR}$ subtypes and are rarely detected in $\alpha 3^{*} \mathrm{nAChRs}$ (Gotti et al., 2007; Perry et al., 2007; Kuryatov et al., 2008; 
Mao et al., 2008). Moreover, $\alpha 5$ subunits were shown to incorporate far more readily into $\beta 2^{*} \mathrm{nAChRs}$ than $\beta 4^{*}$ $\mathrm{nAChR}$ in the mHb-IPn circuit of rats and mice (Grady et al., 2009). In Xenopus oocytes, only the function of $\alpha 4 \beta 2 \alpha 5^{*}$ nAChRs but not $\alpha 3 \beta 4 \alpha 5^{*}$ or $\alpha 3 \beta 2 \alpha 5^{*}$ nAChRs was affected by incorporation of mutant $\alpha 5$ subunits modified to include the major smoking-related CHRNA5 risk allele (D398N), as measured by agonist-evoked calcium influx (Kuryatov et al., 2011). This suggests that $\alpha 4 \beta 2 \alpha 5^{*}$ nAChRs likely play a major role in regulating the stimulatory actions of nicotine on the $\mathrm{mHb}$-IPn circuit. However, as noted above, pharmacological blockade of $\alpha 3 \beta 4^{*} \mathrm{nAChRs}$ attenuated the stimulatory effects of nicotine on the mHb-IPn circuit and increased nicotine intake in rats (Glick et al., 2011; Elayouby et al., 2021), whereas overexpression of these receptors in the mHb-IPn circuit increased nicotine aversion and decreased nicotine intake in mice (Frahm et al., 2011; Slimak et al., 2014). Hence, both $\alpha 4 \beta 2 \alpha 5^{*}$ and $\alpha 3 \beta 4^{*}$ nAChRs in the mHb-IPn circuit appear to regulate nicotine aversion. Nicotine enhances both glutamate and acetylcholine release from habenular terminals in the IPn (Ren et al., 2011), with both neuro-transmitters coreleased from the same $\mathrm{mHb}$ cholinergic neurons (Ren et al., 2011). Acetylcholine also facilitates the packaging of glutamate into synaptic vesicles in $\mathrm{mHb}$ cholinergic neurons, thereby indirectly facilitating glutamatergic transmission in the IPn (Frahm et al., 2015). Discrete firing patterns of habenular cholinergic neurons are required to stimulate glutamate versus acetylcholine release (Ren et al., 2011), with brief optogenetic stimulation sufficient to elicit glutamate release but more persistent (tetanic) optogenetic stimulation required to trigger acetylcholine release (Ren et al., 2011). Intriguingly, $\alpha 3 \beta 4^{*} \mathrm{nAChRs}$ are thought to exclusively regulate acetylcholine release in the $\mathrm{mHb}-\mathrm{IPn}$ circuit (Grady et al., 2001; Hussain et al., 2008; Grady et al., 2009), whereas $\alpha 4 \beta 2 \alpha 5^{*} \mathrm{nAChRs}$ regulate glutamate transmission (Girod et al., 2000). These findings suggest that multiple parallel yet pharmacologically distinct streams of information originate from habenular inputs to the IPn. The first is a glutamate-regulated signal modulated by $\alpha 4 \beta 2 \alpha 5^{*} \mathrm{nAChRs}$, and the second is an acetylcholine-regulated signal modulated by $\alpha 3 \beta 4^{*}$ nAChRs. In addition, a third stream of information is likely to originate from substance $\mathrm{P}$-expressing neurons in dorsal portions of the $\mathrm{mHb}$ that project to the lateral IPn (see below), but remarkably little is known about the function of these substance $P$ cells. How these putatively dissociable signals function together and independently to regulate the motivational properties of nicotine is unknown.

\section{G. nAChRs in IPn Regulate Nicotine Reward and Aversion}

The $\mathrm{mHb}$ projects almost exclusively to the IPn via the fasciculus retroflexus (Qin and Luo, 2009), where
$\mathrm{mHb}$ neurons corelease glutamate and acetylcholine (Ren et al., 2011). Nevertheless, relatively little is known about how IPn neurons regulate behavioral responses to nicotine. Aversive doses of nicotine decrease then increase locomotor activity of rats in a time-dependent manner (Morrison and Stephenson, 1972; Stolerman et al., 1973; Clarke and Kumar 1983a,b). Locomotor depression occurs during the first 15-20 minutes after administration of higher nicotine doses followed by locomotor stimulation that can persist for $>60$ minutes. The locomotor-depressing effects of nicotine are thought to reflect nicotine-induced malaise and are mediated at least partly by $\mathrm{mHb}$ cholinergic neurons (Salas et al., 2004a; Frahm et al., 2015; Antolin-Fontes et al., 2020). Excitotoxic lesion of the IPn attenuated the initial locomotor-depressing but enhanced the later locomotor-stimulating effects of nicotine (Hentall and Gollapudi, 1995). This suggests that stimulation of excitatory inputs from the $\mathrm{mHb}$ to the IPn and direct actions on IPn neurons (Mulle et al., 1991; Lena et al., 1993) contributes to aversive behavioral responses to nicotine. Immunoprecipitation techniques have established that significant populations of $\beta 2^{*}$ nAChRs that combine with $\alpha 3$ or $\alpha 4$ subunits and sparse populations of $\beta 2 * \mathrm{nAChRs}$ that combine with $\alpha 6$ subunits are contained within the IPn (Grady et al., 2009). High concentrations of $\beta 4^{*}$ nAChRs are also detected in the IPn, with the majority containing $\alpha 3$ subunits and smaller populations containing $\alpha 4$ or $\alpha 6$ subunits (Grady et al., 2009). As noted above, knockdown of $\alpha 3$ nAChR subunits or pharmacological blockade of $\alpha 3 \beta 4^{*}$ nAChRs in the IPn increased nicotine self-administration in rats (Glick et al., 2011; Elayouby et al., 2021). However, blockade of $\alpha 3 \beta 4^{*} \mathrm{nAChRs}$ in the VTA located bilaterally to the IPn had no effects on nicotine self-administration in rats (Glick et al., 2011). This suggests that $\alpha 3 \beta 4^{*}$ nAChRs expressed by IPn neurons regulate the motivational properties of nicotine. High concentrations of $\alpha 5^{*}$ nAChRs are detected in the IPn (Morton et al., 2018), particularly in rostral, central, and the intermediate regions that receive input from ventral portions of the $\mathrm{mHb}$ (Fonck et al., 2009; Shih et al., 2014; Quina et al., 2017). Optogenetic stimulation of $\alpha 5$ nAChR-expressing neurons in the IPn elicits aversionrelated behaviors in mice, with these behaviors facilitated by pretreatment with nicotine (Morton et al., 2018). Translating ribosomal affinity profiling combined with RNA sequencing has been used to transcriptionally profile $\alpha 5^{*}$ nAChR-expressing $\left(\alpha 5^{+}\right)$neurons in the IPn (Ables et al., 2017). This analysis identified at least two transcriptionally dissociable and spatially segregated populations of $\alpha 5^{+}$neurons (Ables et al., 2017). $\alpha 5^{+}$neurons that express Amigo1 ( $\alpha 5^{\text {Amigo1 }}$ cells) also coexpress high concentrations of somatostatin and nitric oxide synthase 1 , are concentrated rostral nucleus of the IPn, and 
send efferent input to the median raphe and LDTg (Ables et al., 2017). By contrast, $\alpha 5^{+}$neurons that express $E p y c$ ( $\alpha 5^{\text {Epyc }}$ cells) are concentrated in the intermediate nucleus but also present in the rostral nuclei of the IPn and do not send long-range projections but instead are scattered locally within the IPn and to a lesser extent in the raphe nuclei (Ables et al., 2017). Amigo1 and Epyc encode cell adhesion proteins, the function is poorly understood, but their expression is useful for distinguishing between these subpopulations of $\alpha 5^{+}$neurons in IPn. As might be expected, $\alpha 5^{\text {Amigo1 }}$ cells concentrated in the rostral IPn, which receives dense input from the ventrolateral $\mathrm{mHb}$, showed robust increases in activity in response to nicotine treatment (Ables et al., 2017). $\alpha 5^{\text {Epyc }}$ cells located in the rostral IPn also showed nicotine-induced increases in activity, whereas those located in the intermediate nucleus were relatively insensitive to nicotine (Ables et al., 2017). This suggests that $\alpha 5^{\text {Epyc }}$ cells are a collection of at least two further subpopulations of $\alpha 5^{+}$neurons. Excitatory inputs to the IPn from $\mathrm{mHb}$ cholinergic were inhibited by nitric oxide-mediated retrograde signaling presumably derived from the $\alpha 5^{\text {Amigo1 }}$ cells (Ables et al., 2017). Furthermore, expression of a genetically encoded toxin to inhibit neurotransmitter release and thereby silence $\alpha 5^{\text {Amigo1 }}$ cells attenuated nicotine-induced CPP and decreased oral intake of the drug, whereas silencing of $\alpha 5^{\text {Epyc }}$ cells had no effects of nicotine reward (Ables et al., 2017). shRNA-mediated knockdown of nitric oxide synthase 1 in the IPn also attenuated nicotine reward (Ables et al., 2017). These findings suggest that $\alpha 5^{\text {Amigo1 }}$ cells are activated by nicotine and, presumably, by acetylcholine and glutamate derived from $\mathrm{mHb}$ inputs from cholinergic neurons in ventral $\mathrm{mHb}$, which results in the generation of nitric oxide-mediated inhibitory feedback inhibition onto $\mathrm{mHb}$ inputs to these cells. This feedback inhibition of $\mathrm{mHb}$ inputs by $\alpha 5^{\text {Amigo1 }}$ cells contributes to the rewarding properties of nicotine. It is notable that the rewarding and not the aversive properties of nicotine were attenuated by silencing of $\alpha 5^{\text {Amigo } 1}$ cells (Ables et al., 2017), a finding similar to the attenuated nicotine reward seen in mice after genetic deletion of choline acetyltransferase in $\mathrm{mHb}$ cholinergic neurons (Frahm et al., 2015). This suggests that the mHb-IPn circuit not only regulates nicotine aversion but also nicotine reward. The fact that only the aversive properties of nicotine were in impacted in $\alpha 5 \mathrm{nAChR}$ subunit knockout mice suggests that $\alpha 5^{*} \mathrm{nAChR}$-independent signaling mechanisms in the $\mathrm{mHb}-\mathrm{IPn}$ circuit regulate nicotine reward (Jackson et al., 2010; Fowler et al., 2011, 2013; Grieder et al., 2017). In addition to $\alpha 3^{*}$ and $\alpha 5^{*}$ nAChRs, the IPn also contains the highest concentrations of $\alpha 2 \mathrm{nAChR}$ subunit transcripts in the rodent brain (Wada et al., 1988). Allelic variation in CHRNA2, the gene that encodes the $\alpha 2 \mathrm{nAChR}$ subunit, is associated with increased vulnerability to physical dependence on nicotine (Wang et al., 2014) and cannabis (Demontis et al., 2019). $\alpha 2 \mathrm{nAChR}$ subunit knockout mice show higher levels of intravenous nicotine self-administration during the acquisition phase when nicotine-taking behavior is being established but not after stable intake has been established (Lotfipour et al., 2013). In the IPn, $\alpha 2 \mathrm{nAChRs}$ incorporate into in $\beta 2 * \mathrm{nAChRs}$ primarily (Grady et al., 2009). Hence, $\alpha 2 \beta 2 *$ nAChRs in the IPn are likely to regulate the acquisition of nicotine-taking behaviors.

\section{H. Efferent Projections from IPn Regulate Nicotine Reward and Aversion}

Intra-mHb infusion of the $\alpha 3 \beta 4^{*} \mathrm{nAChR}$ antagonists 18-MC or $\alpha$-conotoxin AuIB blocked the stimulatory effects of nicotine but not morphine or $d$ amphetamine on dopamine release in the NAc shell (McCallum et al., 2012). Further, dopamine levels were elevated in the accumbens, and VTA dopamine cells appeared more sensitive to the stimulatory effects of nicotine in $\beta 4 \mathrm{nAChR}$ subunit knockout mice compared with wild-type mice (Harrington et al., 2016). These dopamine-related effects were reversed by virus-mediated re-expression of $\beta 4$ subunits in the IPn of the knockout mice (Harrington et al., 2016). This regulatory action of the mHb-IPn circuit over nicotine-induced increases in accumbal dopamine transmission may explain why manipulations of neurons in the mHb-IPn circuit can modify both the rewarding and aversive properties of nicotine (Jackson et al., 2010; Fowler et al., 2011, 2013; Frahm et al., 2015; Ables et al., 2017; Grieder et al., 2017). Unlike the $\mathrm{LHb}$, the $\mathrm{mHb}$ does not provide any direct projections to midbrain dopamine neurons, nor does it project to the accumbens (Aizawa et al., 2012; Viswanath et al., 2014). Similarly, IPn neurons do not send projections to the VTA (Lima et al., 2017; Quina et al., 2017; Metzger et al., 2019). How then does the $\mathrm{mHb}-\mathrm{IPn}$ circuit regulate dopamine transmission and the reward effects of nicotine? Recent findings have begun to shed some light on this issue. The IPn sends inhibitory and excitatory inputs from anatomically segregated subnuclei to the LDTg (Lima et al., 2017; Quina et al., 2017), a component of which arises from the $\alpha 5^{\text {Amigo1 }}$ GABAergic neurons in rostral IPn (Ables et al., 2017) (Fig. 5). IPn inputs to LDTg are predominately GABAergic, as expected considering the majority of IPn neurons synthesize GABA. However, a small component of IPn input to the LDTg is glutamatergic, likely reflecting efferent projections from the sparse populations of neurons in IPn that synthesize glutamate (Quina et al., 2017). The LDTg sends reciprocal GABAergic and cholinergic projections to the IPn (Bueno et al., 2019). Nicotine acts at presynaptic terminals to enhance GABAergic and glutamatergic transmission in the LDTg (Ishibashi et al., 2009), with at least a portion of these responses 


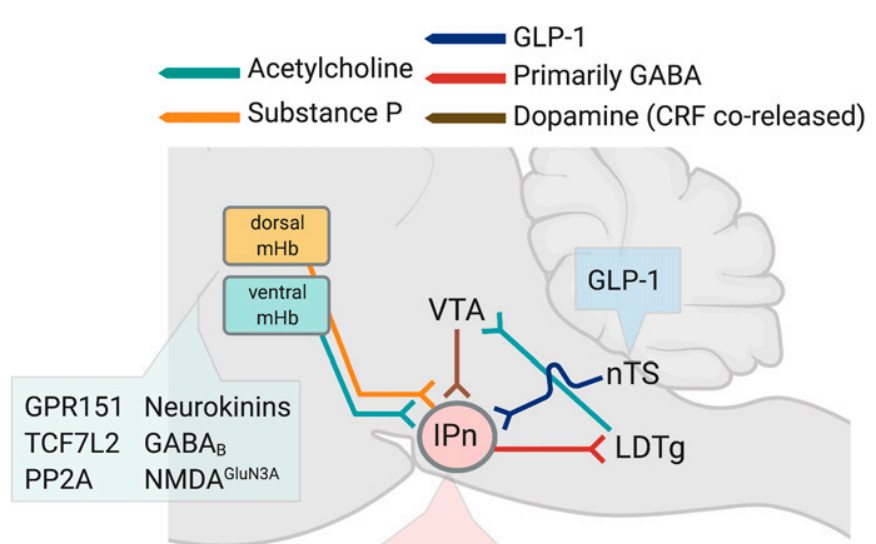

Glycine

Nitric oxide

Endocannabinoids

Fig. 5. Habenula-interpeduncular mechanisms of nicotine aversion. Neurons in dorsal and ventral $\mathrm{mHb}$ express genes implicated in nicotine aversion and other aversion-related behavioral states. These genes include the orphan G-protein coupled receptor (GPCR) GPR151, the transcription factor TCF7L2, the phosphatase PP2A, neurokinins and their receptors, GABA $_{B}$ receptors, and NMDA receptors that contain NR3A subunits. The IPn receives inputs from the nTS that release the neuropeptide GLP1, which facilitates excitatory transmission in the IPn and thereby enhances nicotine aversion. The IPn also receives inputs from VTA neurons that release dopamine and CRF, both of which increase IPn neural activity. Excitatory transmission in the IPn derived from excitatory inputs is regulated by locally released glycine, nitric oxide, and endocannabinoids. The IPn sends an inhibitory projection to the LDTg that inhibits cholinergic projections from the LDTg to the VTA, which results in decreased activity of VTA dopamine neurons. See main text for further details.

presumably reflecting the actions of nicotine on IPn terminals in the LDTg. The LDTg also sends cholinergic projections to the VTA that regulate the tonic and phasic activity of local dopamine neurons through LDTg-derived cholinergic transmission acting act $\beta 2 *$ nAChRs (Lodge and Grace, 2006; Mameli-Engvall et al., 2006; Chen and Lodge, 2013; Faget et al., 2016) (Fig. 5). Hence, LDTg cholinergic projections to the VTA control VTA-derived dopamine release in the accumbens (Blaha et al., 1996; Forster and Blaha, 2000). LDTg cholinergic, GABAergic, and glutamatergic afferents can also bypass the VTA to directly innervate the accumbens (Dautan et al., 2014; Coimbra et al., 2019). These LDTg inputs to the VTA and accumbens play major roles in controlling rewardand aversion-related behaviors (Dautan et al., 2016; Xiao et al., 2016; Steidl et al., 2017b; Coimbra et al., 2019) in a manner that depends on accumbal dopamine transmission (Steidl and Veverka, 2015; Steidl et al., 2017a). Moreover, the LDTg-VTA circuit is known to regulate behavioral responses to nicotine (Alderson et al., 2005; Maskos, 2008; Ishibashi et al., 2009). Hence, the MHb-IPn circuit may influence mesoaccumbens dopamine transmission through an indirect mechanism that involves IPn projections to the LDTg. Consistent with this possibility, nicotine stimulates $\beta 2^{*} \mathrm{nAChRs}$ on IPn neurons to increase inhibitory GABAergic transmission in LDTg neurons (Wolfman et al., 2018). Moreover, optical stimulation of IPn terminals in the LDTg elicited avoidance behaviors, whereas optical inhibition of the IPn-LDTg circuit attenuated nicotine aversion (Wolfman et al., 2018). These findings suggest that an mHb-IPnLDTg-VTA circuit regulates nicotine reward and aversion, in part by modulating mesoaccumbens dopamine transmission.

In addition to the LDTg and raphe nuclei, the IPN also projects to other hindbrain sites, including the dorsolateral tegmental nucleus and nucleus incertus (Smaha and Kaelber, 1973; Shibata and Suzuki, 1984; Goto et al., 2001; Olucha-Bordonau et al., 2003). The IPn also provides ascending projections to septal and hippocampal sites, although whether IPn neurons are the source of these ascending projections has been questioned (Quina et al., 2017). There is a small population of serotonergic (5-hydroxytryptophan) neurons in apical regions of the IPn (IPn ${ }^{5 \mathrm{HT}}$ cells) known to provide ascending input to the hippocampus and septum (Groenewegen and Steinbusch, 1984; Sherafat et al., 2020), which are activated by aversive stimuli (Chen et al., 2003). Chemogenetic inhibition of the $\mathrm{IPn}^{5 \mathrm{HT}}$ cells that project to ventral hippocampus (IPn ${ }^{5 \mathrm{HT}}-\mathrm{VH}$ cells) enhanced escape behaviors and increased sucrose intake in mice (Sherafat et al., 2020). However, chemogenetic inhibition of $\mathrm{IPn}^{5 \mathrm{HT}}$ VH cells did not alter responding for intravenous nicotine infusions in mice even when a higher unit dose of nicotine on the descending portion of the doseresponse curve was available (Sherafat et al., 2020). This suggests that $\mathrm{IPn}^{5 \mathrm{HT}}-\mathrm{VH}$ cells are not involved in regulating nicotine intake, although it is unclear whether projections from $\mathrm{IPn}^{5 \mathrm{HT}}$ cells to other addiction-relevant brain sites may be involved. It is important to note that the apical portion of the IPn, which contains serotonergic and other ascending projections to limbic brain regions is functionally and anatomically distinct from the IPn proper (Quina et al., 2017). It has been argued that this apical region may in fact represent an extended portion of the median raphe nucleus (Quina et al., 2017). If so, the serotonergic neurons in apical IPn regions may not be participate in behaviors directly regulated by the mHb-IPn circuit. As noted above, the IPn sends dense projections to the nucleus incertus in the hindbrain. Indeed, it was shown that $\alpha 5^{+}$IPn neurons send GABAergic projections to the nucleus incertus (Morton et al., 2018), and the sparse population of IPn glutamatergic neurons also project to this site (Quina et al., 2017). Nucleus incertus neurons in turn project to the hippocampus and elsewhere in the brain (Goto et al., 2001; Szőnyi et al., 2019). Optogenetic stimulation of nucleus incertus neurons that project to hippocampus blocked the encoding of fear memories in mice, whereas their 


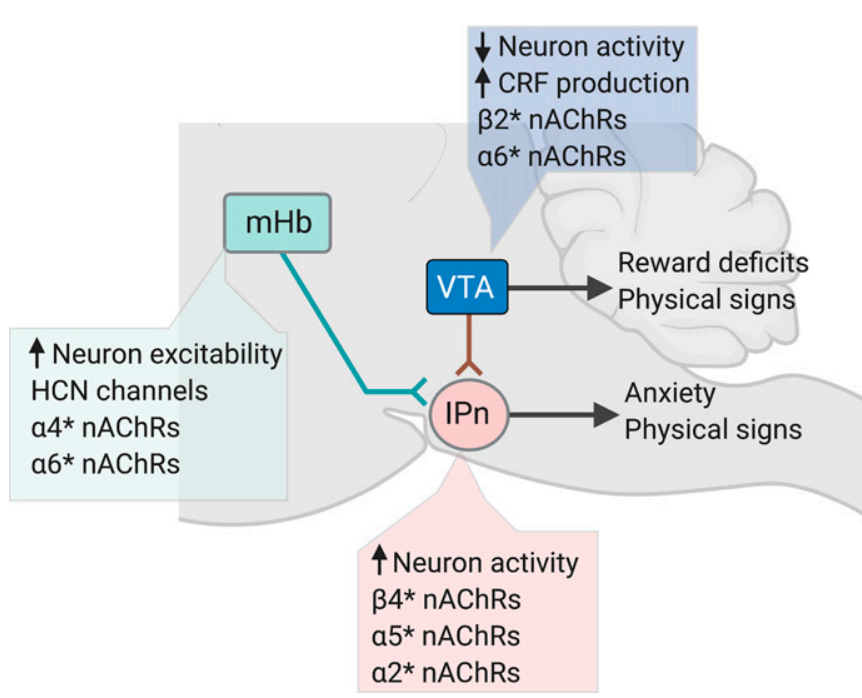

Fig. 6. VTA, habenula, and interpeduncular nucleus contributions to nicotine withdrawal. Neurons in ventral $\mathrm{mHb}$ show increased excitability after chronic nicotine treatment, and inhibitors of $\mathrm{HCN}$ pacemaker channels can precipitate withdrawal in nicotine-dependent animals. In addition, $\alpha 4^{*}$ and $\alpha 6^{*} \mathrm{nAChR}$ function in the $\mathrm{mHb}$ is upregulated in nicotinedependent animals. In the IPn, nicotine withdrawal is associated with increased activity of local GABAergic neurons that express somatostatin, and upregulated expression/function of $\beta 4^{*}, \alpha 5^{*}$, and $\alpha 2^{*} \mathrm{nAChRs}$ is thought to contribute to the expression of nicotine withdrawal. In the VTA, nicotine withdrawal is associated with decreased activity of dopamine neurons that project to the nucleus accumbens, increased production and release of the stress hormone CRF, and upregulated expression/ function of $\beta 2^{*}, \alpha 6^{*} \mathrm{nAChRs}$.

inhibition markedly enhanced the encoding of fear memories (Szőnyi et al., 2019). This suggests that bidirectional modulation of nucleus incertus activity by inhibitory and excitatory inputs from the IPn potentially contributes to the rewarding and aversive actions of nicotine, respectively, a possibility that has not yet been explored.

\section{Afferent Projections to IPn Regulate Nicotine Reward and Aversion}

The IPn receives inputs from brain sites other than the $\mathrm{mHb}$ known to encode reward and aversion states and likely to influence behavioral responses to nicotine. Recent evidence suggests that dopamine neurons in the VTA project to the IPn (VTA-IPn neurons) (Molas et al., 2017; DeGroot et al., 2020) (Fig. 5). Optogenetic stimulation of VTA-IPn neurons decreased anxiety-related behaviors in mice (DeGroot et al., 2020). Similarly, infusion of a D1 dopamine receptor agonist into the IPn decreased anxiety-related behaviors whereas a D1 receptor antagonist had the opposite effect (DeGroot et al., 2020). Hence, nicotine acting on VTA dopamine neurons that project to the IPn, potentially through $\alpha 5^{*}$ nAChRs expressed by these cells, may contribute to its well known anxiety-promoting actions and other aversive responses that promote nicotine avoidance (File et al., 1998, 2000; Ouagazzal et al., 1999a,b; Kenny et al., 2000). In addition to the mHb-IPn circuit, $\alpha 5, \alpha 3$, and $\alpha 4$
nAChR subunit transcripts are also densely expressed by neurons in the nTS. Nicotine dose-dependently activates nTS neurons, as reflected by increases in $\mathrm{cFos}$ immunoreactivity, with this effect most apparent in nTS neurons that express the neuropeptide glucagon-like peptide-1 (GLP-1) (Tuesta et al., 2017). Using a line of Chrna5-tdTomato reporter mice, GLP-1 neurons were shown to express $\alpha 5 \mathrm{nAChR}$ subunits (Tuesta et al., 2017). Systemic administration of the GLP-1 receptor agonist exendin- 4 or the dipeptidyl peptidase 4 inhibitor sitagliptin, which inhibits GLP-1 breakdown, decreased nicotine self-administration in mice (Tuesta et al., 2017). Using transgenic mice that express Cre recombinase in GLP-1 neurons in nTS, it was shown that chemogenetic stimulation of these cells reduced nicotine self-administration (Tuesta et al., 2017). Conversely, GLP-1 receptor knockout mice self-administered greater quantities of nicotine than their wild-type littermates (Tuesta et al., 2017). Some of the highest densities of GLP-1 receptor binding sites in the brain are detected in the IPn (Goke et al., 1995), suggesting that GLP-1-expressing nTS neurons project to the IPn and thereby regulate nicotine intake. Indeed, GLP-1-immunoreactive fibers were detected in the IPn of mice. Furthermore, optical stimulation of the terminals of GLP-1 neurons in the IPn enhanced local excitatory transmission through a mechanism involving increased glutamate release from $\mathrm{mHb}$ inputs (Tuesta et al., 2017) (Fig. 5). Consistent with this mechanism of action, infusion of exendin-4 into the IPn or habenula decreased nicotine intake, whereas IPn infusion of the GLP-1 receptor antagonist exendin-(9-39)amide or shRNA-mediated knockdown of GLP-1 receptor mRNA in the mHb-IPn circuit increased nicotine selfadministration in rats (Tuesta et al., 2017). GLP-1 neurons are known to be activated by gastric expansion to induce feelings of satiety and trigger cessation of food consumption (van Bloemendaal et al., 2014). GLP-1 neurons also regulate feelings of malaise and nausea when food is consumed past satiety (Chang and Scott, 1984; Hayama et al., 1985; Scott et al., 1986; Garcia-Diaz et al., 1988; Monnikes et al., 1997; Willing and Berthoud, 1997; Rinaman et al., 1998; Gutzwiller et al., 1999; Schwartz, 2000; Qin et al., 2005; Appleyard et al., 2007; Bello and Moran, 2008). Hence, it was proposed that activation of GLP-1 neurons by nicotine engages IPn circuits to elicit satiety-like responses to the drug that promote nicotine avoidance behaviors and thereby terminate nicotine selfadministration (Tuesta et al., 2017).

\section{J. Other Reward and Aversion Signaling Mechanisms in the $\mathrm{mHb}-\mathrm{IPn}$ Circuit}

In addition to nAChRs, other receptors and signaling mechanisms in the mHb-IPn circuit have been shown to regulate behavioral responses to nicotine. As described above, GLP-1 facilitates excitatory transmission in the $\mathrm{mHb}-\mathrm{PN}$ circuit to promote nicotine avoidance behaviors (Tuesta et al., 2017). GLP-1 receptors 
are G-protein-coupled receptors (GPCRs) that, when activated, enhance the production of cAMP. In pancreatic $\beta$ cells, GLP-1-enhanced cAMP signaling results in the phosphorylation and nuclear translocation of $\beta$-catenin, which dimerizes with the transcription factor TCF7L2, and TCF7L2 is considered a core component the GLP-1 signaling cascade (Yi et al., 2005; Liu and Habener, 2008; Vazquez-Roque et al., 2011; Chiang et al., 2012; Shao et al., 2013). TCF7L2 is densely expressed in $\mathrm{mHb}$ cholinergic neurons (Duncan et al., 2019). Using a line of genetically modified rats that express a loss-of-function TCF7L2 variant, it was shown that TCF7L2 deficiency markedly reduced aversive behavioral responses to nicotine and increased nicotine self-administration behavior (Duncan et al., 2019). Similarly, CRISPR/CRISPR-associated protein 9-mediated genomic cleavage of the $T c f 7 l 2$ gene in the $\mathrm{mHb}$ of mice or shRNA-mediated knockdown of TCF7L2 mRNA transcripts in the $\mathrm{mHb}$ of rats increased nicotine self-administration behavior (Duncan et al., 2019). The stimulatory effects of nicotine on mHb inputs to the IPn were diminished in the TCF7L2-deficient rats, as reflected by reductions in $\mathrm{mHb}$-derived excitatory transmission in IPn slices evoked by nicotine (Duncan et al., 2019). The diminished sensitivity of the mHb-IPn circuit to nicotine was explained by a deficit in the function but not the expression of $\mathrm{nAChRs}$ in $\mathrm{mHb}$ neurons (Duncan et al., 2019). Deficient TCF7L2-mediated transcriptional activity in the $\mathrm{mHb}$ of the TCF7L2 mutant rats resulted in striking deficits in the production of cAMP in $\mathrm{mHb}$ neurons, which reduced activity of the cAMP-regulated protein kinases that positively regulate nAChR function in mHb neurons (Huganir et al., 1986; Paradiso and Brehm, 1998; Giniatullin et al., 2005). This abnormality in cAMP signaling explained the deficits in habenular nAChR function and the elevated nicotine intake seen in TCF7L2-deficient animals (Duncan et al., 2019). These findings highlight the importance of cAMP and likely other intracellular signaling processes that regulate $\mathrm{nAChR}$ function in the $\mathrm{mHb}-\mathrm{IPn}$ pathway in the reinforcing actions to nicotine.

GPR151 is orphan GPCR that is highly enriched in habenular neurons in rodents and humans, particularly $\mathrm{mHb}$ neurons that coexpress $\alpha 3^{*}$ nAChRs (Quina et al., 2009; Kobayashi et al., 2013; Broms et al., 2015; Wagner et al., 2016; Broms et al., 2017; Antolin-Fontes et al., 2020). The stimulatory effects of nicotine on $\mathrm{mHb}$ neurons are attenuated in GPR151 knockout mice (Antolin-Fontes et al., 2020). The knockout mice also had attenuated aversive behavioral responses to nicotine and self-administered greater numbers of intravenous nicotine infusions than wild-type mice, particularly when a higher unit dose on the descending portion of the dose-response curve was available (Antolin-Fontes et al., 2020). GPR151 appears to be negatively coupled to adenylyl cyclase activity, as levels of cAMP were elevated in $\mathrm{mHb}$ tissue collected from GPR151 knockout mice compared with wild-type mice (Antolin-Fontes et al., 2020). Hence, GPR151 may modulate behavioral responses to nicotine in a manner similar to TCF7L2 by regulating the cAMP-dependent kinases that control nAChR function in the mHb-IPn circuit. GPR151 is thought to be expressed almost exclusively on the terminals of $\mathrm{mHb}$ neurons in the IPn (Antolin-Fontes et al., 2020). Hence, GPR151 may influence nicotine intake by regulating the function of presynaptically located nAChRs on $\mathrm{mHb}$ terminals and the neurotransmitter release machineries in $\mathrm{mHb}$ terminals known to be controlled by cAMP-dependent signaling processes (Azhderian et al., 1994; Chen and Regehr, 1997; Duncan et al., 2019).

GPR139 is another orphan GPCR that is densely expressed in the $\mathrm{mHb}$ and is also found in the $\mathrm{LHb}$, septum, and striatum (Liu et al., 2015; Wagner et al., 2016). Allelic variation in the GPR139 gene influences body mass index and blood pressure in a manner that depends on smoking status (current smoker, ex-smoker, or nonsmoker) (Justice et al., 2017; Sung et al., 2018). GPR139 modulates $\mu$ opioid receptor function in the $\mathrm{mHb}$ to regulate the rewarding actions of opioids (Wang et al., 2019) through a mechanism that likely involves regulation of cAMP signaling dynamics in $\mathrm{mHb}$ neurons (Stoveken et al., 2020). The role for GPR139 in regulating the motivational properties of nicotine has not yet been explored. However, the novel GPR139 agonist TAK-041 blocks the stimulatory effects of nicotine on accumbal dopamine release (Schiffer et al., 2020), suggesting that GPR139 located in the mHb-IPn circuit and striatum may modulate the rewarding and aversive actions of nicotine, a possibility that awaits further investigation.

Phosphodiesterase 2A (PDE2A) metabolizes cAMP and is robustly expressed by neurons in the $\mathrm{mHb}$ and IPn as well as the hippocampus, striatum, globus pallidus, and substantia nigra (Stephenson et al., 2009, 2012). Atrial natriuretic peptide activates PDE2A in $\mathrm{mHb}$ neurons, thereby inhibiting the release of glutamate into the IPn (Hu et al., 2012). This inhibitory action of atrial natriuretic peptide on $\mathrm{mHb}$-derived glutamatergic transmission in the IPn was prevented by PDE2A inhibition or by a chemical activator of cAMPregulated protein kinase A (Hu et al., 2012). These findings further highlight the importance of cAMP signaling in regulating $\mathrm{mHb}$-IPn circuit function and suggest that PDE2A modulators that influence $\mathrm{mHb}-\mathrm{IPn}$ circuit responses to nicotine may represent a novel class smoking-cessation therapeutics.

As described above, $\alpha 5 \beta 2^{*}$ nAChRs enhance neurokinin signaling in the $\mathrm{mHb}$, which increases the intrinsic excitability of $\mathrm{mHb}$ neurons (Dao et al., 2014). The source of neurokinins in this action is unclear but may 
derive from noncholinergic neurons in dorsal $\mathrm{mHb}$ that synthesize substance $\mathrm{P}$ (neurokinin $\mathrm{A}$ ) and project to lateral IPn (Contestabile et al., 1987; Qin and Luo, 2009; Aizawa et al., 2012; Melani et al., 2019). Substance $\mathrm{P}$-expressing $\mathrm{mHb}$ neurons in dorsal $\mathrm{mHb}$ that project to lateral IPn also express $\mathrm{CB}_{1}, \mathrm{GABA}_{\mathrm{B}}$, and glycine receptors, all of which regulate the encoding or extinction of fear memories (Soria-Gomez et al., 2015; Zhang et al., 2016; Melani et al., 2019) (Fig. 5). Stimulation of neurons in dorsal $\mathrm{mHb}$ neurons increased excitatory glutamatergic transmission in the lateral IPn, which in turn stimulated local inhibitory glycine-containing neurons in the IPn (Melani et al., 2019) (Fig. 5). Glycine released in this manner is thought to serve a negative feedback role by counterbalancing the local actions of $\mathrm{mHb}$-derived glutamate and thereby opposing glutamate-driven long-term synaptic potentiation in the IPn (Melani et al., 2019). Strychnine-mediated inhibition of glycinergic transmission in lateral IPn disrupted this counter-regulatory response and enhanced the release of glutamate from $\mathrm{mHb}$ terminals, which in turn facilitated the induction of long-term synaptic potentiation at the dorsal $\mathrm{mHb}$-lateral IPn synapse (Melani et al., 2019). This synaptic potentiation was blocked by neurokinin 1 receptor antagonism (Melani et al., 2019), suggesting that glycine released from lateral IPn neurons acts by inhibiting substance $\mathrm{P}$ and glutamate corelease from $\mathrm{mHb}$ terminals in the IPn (Melani et al., 2019). Injection of a neurokinin 1 receptor antagonist into lateral IPn, which blocked the actions of substance $\mathrm{P}$ released by dorsal $\mathrm{mHb}$ inputs, impaired the ability of mice to extinguish fear memories (Melani et al., 2019), suggesting that dorsal mHb-lateral IPn synapse controls the processing and gradual extinction of behavioral responses to aversive stimuli. Notably, $\mathrm{CB}_{1}$ receptor antagonism also blocked activitydependent synaptic potentiation at the dorsal mHb-lateral IPn synapse, an effect mimicked by a $\mathrm{GABA}_{\mathrm{B}}$ receptor antagonist (Melani et al., 2019) (Fig. 5). Conditional deletion of $\mathrm{CB}_{1}$ receptors from $\mathrm{mHb}$ neurons decreased fear-conditioned freezing behavior and abolished conditioned odor aversion in mice but did not alter behavioral responses to neutral or appetitive stimuli Soria-Gomez et al. (2015). These findings suggest that glycine, endocannabinoid, and neurokinin transmission in the IPn regulate the acquisition, expression, and extinction of behavioral responses to aversive stimuli (Fig. 5). Interestingly, $\mathrm{mHb}$ neurons express an uncommon NMDA receptor subtype that contains GluN1/GluN3A subunits, which are activated by glycine (Otsu et al., 2019). Activation of these GluN1/GluN3A NMDA receptors by glycine increases the activity of $\mathrm{mHb}$ neurons, which triggers avoidance-related behaviors (Otsu et al., 2019) (Fig. 5). It is likely that glycine, endocannabinoid, and neurokinin transmission in lateral IPn regulate aversive responses to nicotine in a manner that involves GluN1/GluN3A NMDA receptors on the terminals of $\mathrm{mHb}$ neurons. This possibility has not yet been explored.

\section{K. Nicotine Actions in the Periphery Impact Reward and Aversion Behaviors}

The nTS is the major site to which the vagus nerve (cranial nerve $\mathrm{X}$ ) delivers sensory information to the brain from peripheral organs involved in processing the interoceptive actions of nicotine contained in cigarette smoke (Ogawa et al., 1984; Hines et al., 1994). This raises the possibility that nicotine acts not only at nAChRs located directly in the nTS but also at nAChRs located in peripheral organs, with nicotine-related sensory information contributing to nTS activation and nicotine avoidance behaviors. Indeed, nAChRs are expressed in tissues that come into direct contact with nicotine in tobacco smoke, including nAChRs in the mouth and lungs. Sensory information related to the actions of nicotine in the oral cavity, airways, and elsewhere in the body (Cain, 1980; Ahijevych et al., 2015) activate nTS neurons through vagal inputs (Lemon and Smith, 2005; Simons et al., 2006). Nicotine-related sensory information is then relayed from the nTS to higher-order midbrain and corticothalamic circuits involved in reward and avoidance behaviors. This nicotine-related sensory information contributes to the interoceptive properties of tobacco smoke that smokers use to titrate their intake to avoid its aversive properties (Iwasaki and Sato, 1981; Feyerabend et al., 1982; Rose et al., 1993; Pritchard et al., 1996; Dahl et al., 1997; Ming et al., 1998; Fowler and Kenny, 2014). Nicotine acting peripherally can also precipitate craving in smokers during periods of abstinence (Naqvi et al., 2007; Gray and Critchley, 2007; Naqvi and Bechara, 2010; Fowler and Kenny, 2014). Moreover, blocking the sensory properties of nicotine in cigarette smokers attenuates the rewarding properties of cigarettes and decreases smoking behavior (Rose et al., 1985, 1993, 2016; McClernon et al., 2016). In rodents, actions of nicotine outside the brain contribute to many of the behavioral and physiologic responses to the drug (Kiyatkin, 2014). Despite the considerable evidence linking peripheral actions of nicotine to tobacco use disorder, remarkably little is known about the underlying mechanisms of such brain-body interactions. However, a number of groups have used the modified nicotine analog nicotine pyrrolidine methiodide (nicotine-PM), which is a full nAChR agonist that does not cross the blood-brain barrier (Lenoir and Kiyatkin, 2011; Kiyatkin, 2014) to map brain circuits and cells activated by nicotine acting at peripheral organs (Dehkordi et al., 2015; Rose et al., 2016). It was found that nicotine and nicotine-PM increased cFos immunoreactivity in overlapping brain regions, including the IPn and other sites involved in the reward and aversion, such as the LDTg, PPTg, LHb, accumbens, and VTA (Dehkordi et al., 2015; Rose et al., 2016). Within the VTA, GABAergic but not dopaminergic 
cells were almost exclusively activated by a higher aversive but not by a lower rewarding dose of nicotine (Dehkordi et al., 2018), suggesting that these cells may transduce aversive sensory properties of peripherally acting nicotine. Further studies will be required to explore this possibility and to determine whether nTS inputs to VTA IPn, LDTg, PPTg, LHb, or accumbens explain their sensitivity to the peripheral actions of nicotine.

\section{Mechanisms of Nicotine Withdrawal}

\section{A. Nicotine Withdrawal Syndrome in Humans and Rodents}

Tobacco use disorder depends not only on the rewarding properties of nicotine that underlie its positive reinforcing properties but also on escape from the aversive consequences of nicotine withdrawal that motivate tobacco smoking according to negative reinforcement processes (Doherty et al., 1995; Kenny and Markou, 2001). Chronic nicotine exposure results in the development of a dependent state in smokers such that cessation of intake elicits an aversive nicotine withdrawal syndrome (Shiffman and Jarvik, 1976; Hughes et al., 1991). This withdrawal syndrome is attenuated by nicotine replacement therapy (Schneider and Jarvik, 1984; Fagerstrom et al., 1993; Molander et al., 2000), confirming that nicotine plays a major role in its induction and expression. Conversely, smoking cigarettes with reduced nicotine content can elicit a withdrawal syndrome in smokers that is accompanied by a reduction in plasma nicotine levels (West et al., 1984). The duration and severity of the nicotine withdrawal syndrome in abstinent smokers accurately predict their likelihood of relapse to tobacco use (Piasecki et al., 1998, 2000, 2003). Conversely, the efficacy of nicotine replacement therapy in certain individuals is related to its ability to prevent the onset and reduce the duration of nicotine withdrawal (Fagerstrom, 1988; Sachs and Leischow, 1991). Hence, it is important to understand the brain mechanisms that regulate nicotine withdrawal-associated aversive states.

Nicotine withdrawal in abstinent smokers is comprised of physical and affective components. The most common physical symptoms include bradycardia and gastrointestinal discomfort, suggesting that autonomic nervous system function is disrupted in smokers during withdrawal (Niedermaier et al., 1993). Affective symptoms primarily include irritability, depressed mood, anxiety, difficulty concentrating, and craving (Parrott, 1993; Doherty et al., 1995; Kenny and Markou, 2001). Escape from affective components of withdrawal is thought to play a particularly important role in the maintenance nicotine dependence (Koob and Le Moal, 1997; Markou et al., 1998; Kenny and Markou, 2001). Nicotine withdrawal is associated with physical and affective abnormalities in laboratory rodents. Withdrawal from nicotine and other drugs of abuse is thought to reflect compensatory adaptations in the same neurobiological substrates that regulate acute responses to the drug (Koob and Bloom, 1988; Koob and Le Moal, 1997). Thus, it is likely that the same nAChRs that regulate the rewarding and aversive effects of acutely administered nicotine undergo adaptations upon prolonged exposure to nicotine, which contributes to the development of nicotine dependence and the expression of withdrawal-associated physical and affective components of nicotine withdrawal (Kenny and Markou, 2001).

\section{B. $\beta 2 * n A C h R s$ Regulate Affective but Not Physical Components of Nicotine Withdrawal}

Spontaneous withdrawal from nicotine, accomplished by the surgical removal of subcutaneously implanted osmotic minipumps that delivered nicotine continuously for $>7$ days, was associated with the expression of a physical withdrawal syndrome in rats (Malin et al., 1992; Epping-Jordan et al., 1998). Systemic administration of mecamylamine precipitated physical withdrawal signs in nicotine-dependent rats (Watkins et al., 2000). Systemic or intracerebroventricular administration of the nAChR antagonist chlorisondamine, which does not readily cross the blood-brain barrier, also precipitated physical withdrawal signs in nicotine-dependent rats (Watkins et al., 2000). These findings suggest that nAChRs in the brain regulate physical withdrawal from nicotine. They also suggest that peripheral ganglionic nAChRs located outside the brain contribute to the physical withdrawal syndrome. Indeed, expression of physical withdrawal signs in rats undergoing spontaneous nicotine withdrawal were attenuated by the peripherally acting $\mathrm{nAChR}$ agonist tetramethylammonium (Hildebrand et al., 1997). By contrast, $\beta 2^{*}$ nAChR-preferring antagonist $\mathrm{DH} \beta \mathrm{E}$ did not precipitate physical withdrawal signs in nicotine-dependent rats (EppingJordan et al., 1998). This suggests that $\beta 2^{*}$ nAChRs in the brain are unlikely to contribute to physical dependence on nicotine (see Fig. 6).

In addition to a physical syndrome, spontaneous and antagonist-precipitated nicotine withdrawal is also associated with an affective withdrawal syndrome in rats and mice, which can be measured by elevations of ICSS reward thresholds, the induction of a conditioned place avoidance (CPA), decrements in sucrose consumption, increases in anxiety-related behaviors, and other behavioral abnormalities consistent with the manifestation of a negative affective state (see Kenny and Markou, 2001; Fowler et al., 2008). In nicotinedependent rats, spontaneous withdrawal or withdrawal precipitated by mecamylamine or $\mathrm{DH} \beta \mathrm{E}$, elevated ICSS thresholds (Ivanova and Greenshaw, 1997; Epping-Jordan et al., 1998; Watkins et al., 2000; Harrison et al., 
2002). Similarly, systemic administration of chlorisondamine at doses unlikely to cross the blood-brain barrier or its direct intracerebroventricular administration also elevated ICSS thresholds in nicotine-dependent rats (Epping-Jordan et al., 1998; Watkins et al., 2000). This suggests that nAChRs located in brain reward circuits, including $\beta 2^{*} \mathrm{nAChRs}$, are contribute to the development of "affective dependence" on nicotine. Surprisingly, ganglionic nAChRs located outside the brain may also contribute to this process, although very little is known about the contribution of peripherally located nAChRs to affective components of withdrawal.

$\beta 2 * \mathrm{nAChR}$ subunit knockout mice, which are insensitive to the rewarding properties of nicotine (see above), demonstrated a robust physical nicotine withdrawal syndrome (Besson et al., 2006), but withdrawal-induced elevations in ICSS thresholds were absent in these animals (Stoker et al., 2015). Withdrawal-induced increases in anxiety-related behaviors and the establishment of a CPA for an environment paired with antagonist-precipitated nicotine withdrawal were also absent in $\beta 2$ knockout mice but readily apparent in wild-type mice (Jackson et al., 2008). Deficits in fear conditioning typically observed in wild-type mice experiencing nicotine withdrawal were also attenuated in $\beta 2$ knockout mice (Portugal et al., 2008; Raybuck and Gould, 2009). Most recently, it was shown that $\beta 2$ subunit knockout mice were resistant to nicotine withdrawal-induced deficits in sucrose consumption and increases in anxiety-related behaviors (Alkhlaif et al., 2017). Hence, $\beta 2 *$ nAChRs regulate affective but not physical signs of nicotine withdrawal (see Fig. 6).

\section{Other nAChR Subtypes Contribute to Affective Components of Nicotine Withdrawal}

Similar to $\beta 2 \mathrm{nAChR}$ subunit knockout mice, $\beta 3$ and $\alpha 6 \mathrm{nAChR}$ subunit knockout mice showed attenuated sucrose drinking and anxiety-related behavioral abnormalities during nicotine withdrawal, whereas physical withdrawal signs were unaltered in these animals (Alkhlaif et al., 2017; Jackson et al., 2019). Furthermore, an $\alpha 6^{*}$ nAChR-selective antagonist blocked the expression of a nicotine withdrawal-induced CPA and withdrawal-associated increases in anxiety-related behavior but did not modify the expression of physical withdrawal signs in mice (Jackson et al., 2009). These findings suggest that the same $\alpha 6 \beta 2 \beta 3^{*}$ nAChR subtype known to regulate the stimulatory effects of nicotine on striatal dopamine release (see above) is likely to undergo nicotine-induced adaptations in function and expression during chronic nicotine exposure, which contributes to the development of affective but not physical dependence on nicotine. The role for $\alpha 7 \mathrm{nAChRs}$ in affective components of nicotine withdrawal is less clear. Deficits in context-related encoding of fear memories typically seen in mice experiencing nicotine withdrawal were unaltered in $\alpha 7$ knockout mice, whereas similar withdrawal-related cognitive deficits were absent in $\beta 2$ subunit knockout mice (Portugal et al., 2008). The $\alpha 7$ nAChR antagonist MLA did not precipitate a CPA or other affective signs of withdrawal in nicotine-dependent mice (Markou and Paterson, 2001; Jackson et al., 2008). Nor did MLA precipitate withdrawal-related cognitive deficits in nicotine-dependent rats (Shoaib and Bizarro, 2005). The novel $\alpha 7 \mathrm{nAChR}$ partial agonist encenicline did not attenuate nicotine withdrawal-associated cognitive deficits in abstinent human smokers, as reflected by similar deficits in treated and untreated abstinent smokers when tested using the Conners Continuous Performance Task to assess attention and response inhibition and using the N-Back task to measure working memory (Schuster et al., 2018). Also, the $\alpha 7 \mathrm{nAChR}$ agonist ABT107 did not attenuate cognitive deficits (encoding of fear memories) detected in mice undergoing nicotine withdrawal (Yildirim et al., 2015). However, the $\alpha 7 \mathrm{nAChR}$ agonist PNU282987 attenuated nicotine withdrawal-induced increases in anxiety-related behavior in mice (Jackson et al., 2018). Furthermore, the deficits in attention typically seen in wild-type mice undergoing nicotine withdrawal were absent in $\alpha 7$ knockout mice (Higa et al., 2017). Infusion of MLA into the VTA of nicotinedependent rats decreased accumbal dopamine release in a manner similar to mecamylamine infusion (Nomikos et al., 1999), suggesting that $\alpha 7 \mathrm{nAChRs}$ may contribute to deficits in dopamine transmission during nicotine withdrawal (Nomikos et al., 2000). Together, these findings suggest that $\alpha 7 \mathrm{nAChRs}$ may play a role in affective components of nicotine withdrawal, but its precise contributions remain unclear (see Fig. 6).

\section{Dopamine Transmission Contributes to Affective Components of Nicotine Withdrawal}

Consistent with a role for mesoaccumbens dopamine transmission in nicotine withdrawal, extracellular dopamine levels were markedly reduced in rats undergoing nicotine withdrawal compared with nicotine-nave animals (Hildebrand et al., 1998), opposite to the stimulatory effects of acutely administered nicotine on accumbal dopamine release (see above). Nicotine withdrawal-related deficits in accumbens dopamine release are less severe in adolescent than adult rats (Natividad et al., 2010), which may explain the attenuated withdrawal-related behaviors seen in adolescent versus adult rats (O'Dell et al., 2004, 2007). Mecamylamine delivered into the VTA decreased extracellular dopamine levels in the accumbens and precipitated withdrawal-like physical signs in nicotine-dependent but not nicotine-naive control rats (Hildebrand et al., 1999). Similarly, infusion of $\mathrm{DH} \beta \mathrm{E}$ into the VTA (Bruijnzeel and Markou, 2004) but not the central nucleus of the amygdala or bed nucleus of the stria terminalis (Jonkman and Markou, 2006) precipitated withdrawal-associated elevations of ICSS thresholds in nicotine-dependent but not control rats. Hence, the VTA serves as a key 
neuroanatomical substrate in the regulation of affective components of nicotine withdrawal.

In contrast to the decreased dopamine release detected in the NAc during nicotine withdrawal, dopamine levels were dramatically increased in the $\mathrm{mPFC}$ during withdrawal (Carboni et al., 2000a). This effect is similar to the increased cortical dopamine transmission seen in rodents undergoing withdrawal from opioids (Bozarth, 1994; Bassareo et al., 1995; Espejo et al., 2001). Hence, it is unlikely that the reward deficits and other affective components of withdrawal from nicotine and other major drugs of abuse reflect a generalized state of depressed activity of midbrain dopamine neurons. Instead, the activity of some populations of dopamine neurons are decreased, whereas the activity of others is increased. This likely reflects the same type of partitioning of withdrawal-relevant dopamine neurons in the VTA into functional domains as described above for the reward-related actions of nicotine. Hence, it is an interesting possibility that withdrawal from nicotine and other drugs of abuse is associated with decreased activity of VTA ${ }^{\mathrm{LAT}}$ dopamine neurons that project to the NAc shell and increased activity of VTA ${ }^{\mathrm{MED}}$ dopamine neurons that project to the $\mathrm{mPFC}$.

A population of dopamine neurons that express corticotropin-releasing factor (CRF) has been identified in the posterior VTA (Grieder et al., 2014). Chronic nicotine treatment upregulated CRF expression in these VTA dopamine neurons, which blocked the stimulatory effects nicotine on local GABAergic transmission in the VTA (Grieder et al., 2014). Blockade of CRF signaling in the VTA during nicotine withdrawal restored the stimulatory actions of nicotine on VTA GABA transmission and attenuated affective components of nicotine withdrawal in rats (Grieder et al., 2014). Notably, the majority of these CRF-positive neurons were located in posterior regions of the VTA immediately adjacent to the IPn (Grieder et al., 2014). Moreover, the increased CRF mRNA levels detected in the VTA of nicotine-dependent rats coincided with decreased CRF immunoreactivity in the IPn of the same animals (Grieder et al., 2014). This suggests that posterior VTA neurons that synthesize CRF may project to the IPn, a circuit known to regulate anxiety-related behaviors in rodents and release $\mathrm{CRF}$ into the IPn to drive affective components of nicotine withdrawal. Consistent with this possibility, it was shown that increased anxiety-related behaviors in mice during nicotine withdrawal were associated with increased activity of neurons located in the intermediate nucleus of the IPn, in which $\alpha 5^{\text {Epyc }}$ neurons are known to be located (see above) (Zhao-Shea et al., 2015). This effect was regulated by CRF derived from VTA inputs (Zhao-Shea et al., 2015). Furthermore, blockade of CRF transmission in or optogenetic inhibition of $\mathrm{mHb}$ inputs to the intermediate nucleus of the IPn attenuated nicotine withdrawal-induced increases in anxietyrelated behavior (Zhao-Shea et al., 2015). Further supporting an important role for the IPn in nicotine withdrawal-related increases in anxiety-related behavior, it was shown that chronic nicotine treatment increased the sensitivity of nAChRs in the $\mathrm{mHb}$ to nicotine (Pang et al., 2016; Arvin et al., 2019), with habenular $\alpha 4^{*}$ and $\alpha 6^{*} \mathrm{nAChRs}$ located in the ventroinferior portion of the $\mathrm{mHb}$ being particularly sensitive to this action (Shih et al., 2015; Pang et al., 2016). Moreover, pharmacological blockade of $\alpha 4 \beta 2^{*}$ or $\alpha 6 \beta 2^{*}$ nAChRs but not $\alpha 3 \beta 4^{*}$ $\mathrm{nAChRs}$ in the $\mathrm{mHb}$ alleviated nicotine withdrawalrelated increased in anxiety-related behaviors in mice (Pang et al., 2016). Most recently, it was shown that a history of intravenous nicotine self-administration similarly upregulated $\mathrm{nAChR}$ function in the $\mathrm{mHb}$ of rats (Jin et al., 2020). Finally, it was shown that a metabotropic glutamate $2 / 3$ receptor antagonist infused systemically or directly into the VTA attenuated the elevations of ICSS thresholds in rats undergoing spontaneous nicotine withdrawal (Kenny et al., 2003). These findings suggest that adaptive responses in $\beta 2^{*}, \beta 3^{*}$, and $\alpha 6^{*}$ nAChRs in the VTA, leading to alterations in dopamine, $\mathrm{CRF}$, GABA, and glutamate signaling in the accumbens, IPn, and other reward and aversion relevant brain sites, drives the expression of affective but not physical components of nicotine withdrawal.

\section{E. $\beta 4^{*}$ nAChRs Regulate Physical but Not Affective Components of Nicotine Withdrawal}

$\beta 4^{*} \mathrm{nAChRs}$ appear to regulate physical not affective signs of nicotine withdrawal, opposite to the contributions of $\beta 2^{*}$ nAChRs described above. Indeed, $\beta 4$ nAChR subunit knockout mice had decreased physical signs in response to spontaneous or mecamylamine-precipitated nicotine withdrawal (Salas et al., 2004b; Stoker et al., 2012). However, mecamylamine-precipitated nicotine withdrawal elevated ICSS thresholds by a similar magnitude in wild-type and $\beta 4$ subunit knockout mice (Stoker et al., 2012), although the onset of threshold elevations during spontaneous nicotine withdrawal was delayed in $\beta 4$ knockout mice relative to wild-type mice (Stoker et al., 2012). Similar to $\beta 4$ knockout mice, $\alpha 5$ subunit knockout mice also demonstrated a loss of nicotine withdrawal-induced physical withdrawal signs compared with wild-type mice (Jackson et al., 2008; Salas et al., 2009). By contrast, withdrawalinduced increases in anxiety-related behaviors were unaltered in $\alpha 5$ knockout mice (Jackson et al., 2008). The role for $\alpha 2 \mathrm{nAChRs}$ in nicotine withdrawal is complex, however, as one study reported that physical withdrawal signs were attenuated in $\alpha 2 \mathrm{nAChR}$ subunit knockout mice (Salas et al., 2009), and another reported that physical withdrawal was exacerbated in these animals (Lotfipour et al., 2013). The role for $\alpha 7 \mathrm{nAChRs}$ in physical aspects of nicotine withdrawal is also unclear. 
Some studies have shown that physical withdrawal signs were attenuated in $\alpha 7$ subunit knockout mice (Salas et al., 2007), whereas others have shown no difference between knockout and wild-type mice (Stoker et al., 2012). The $\alpha 7 \mathrm{nAChR}$ antagonist MLA did not precipitate physical signs of withdrawal in nicotine dependent mice (Markou and Paterson, 2001; Jackson et al., 2008). However, the $\alpha 7$ nAChR agonist PNU282987 attenuated nicotine withdrawal-induced increases in anxiety-related behaviors and physical withdrawal signs in mice (Jackson et al., 2018). Overall, these findings suggest that $\beta 4^{*}$ and $\alpha 5^{*} \mathrm{nAChRs}$ and perhaps $\alpha 2^{*}$ and $\alpha 7 \mathrm{nAChRs}$ regulate physical dependence on nicotine.

\section{F. nAChR Signaling in mHb-IPn Circuit Regulates Nicotine Withdrawal}

Considering the high concentrations of $\beta 4, \alpha 5$, and $\alpha 2$ nAChR subunits in the mHb-IPn circuit, it is perhaps not surprising that this circuit has been implicated in physical components of the nicotine-withdrawal syndrome. Direct infusion of mecamylamine into the $\mathrm{mHb}$ or IPn but not the cortex, VTA, or hippocampus precipitated physical withdrawal signs and increased anxietyrelated behaviors in nicotine-dependent mice (Salas et al., 2009; Zhao-Shea et al., 2013). Mecamylamine infused into the IPn also increased physical signs of withdrawal and anxiety-related behaviors in nicotinedependent male and female rats (Correa et al., 2019). The onset of nicotine withdrawal was associated with increased activity of IPn GABAergic neurons, as reflected by increased $\mathrm{cFos}$ expression and spontaneous excitatory currents in IPn neurons (Zhao-Shea et al., 2013). Pharmacological blockade of $\mathrm{mHb}$-derived glutamatergic transmission in the IPn attenuated, whereas the optical stimulation of IPn GABAergic neurons precipitated, withdrawal-related physical signs in mice (Zhao-Shea et al., 2013). Chronic nicotine treatment upregulated the expression of $\beta 4 \mathrm{nAChR}$ subunits in somatostatin-expressing IPn neurons in dorsal portions of the IPn (Zhao-Shea et al., 2013), which may reflect the $\alpha 5^{\text {Amigo }}$ cells that regulate nitric oxide-mediated retrograde inhibition of $\mathrm{mHb}$ inputs to the IPn (see above) (Ables et al., 2017). This suggests that adaptions in $\beta 4^{*}$ nAChR signaling in the IPn contribute to the development of nicotine dependence. Consistent with this interpretation, infusion of the $\alpha 3 \beta 4^{*} \mathrm{nAChR}$ antagonist SR 16584 into the IPn of nicotine-dependent mice precipitated physical signs of nicotine withdrawal (Zhao-Shea et al., 2013). Together, these findings suggest that $\beta 4^{*}$ nAChRs and perhaps $\alpha 5^{*}$ and $\alpha 2^{*}$ nAChRs located in the $\mathrm{mHb}$-IPn circuit regulate physical signs of nicotine withdrawal. However, the $\mathrm{mHb}-\mathrm{IPn}$ circuit also regulates the increases in anxiety-related behaviors that occur during nicotine withdrawal, although $\beta 4^{*}, \alpha 5^{*}$, and $\alpha 2^{*}$ nAChRs do not appear to be involved. This may instead reflect the contribution of $\beta 2^{*} \mathrm{nAChR}$ - expressing dopamine neurons in the VTA that express $\mathrm{CRF}$ and project to the IPn to modulate anxiety-related states (Grieder et al., 2014; Zhao-Shea et al., 2015) (see above).

In addition to IPn neurons, $\mathrm{mHb}$ neurons also show adaptive alterations in their activity in response to chronic nicotine exposure, which contribute to the development of nicotine dependence and expression of the aversive nicotine withdrawal syndrome (Gorlich et al., 2013). In slice recordings, $\mathrm{mHb}$ neurons show tonic trains of action potentials (Kim and Chang, 2005; Gorlich et al., 2013), which likely reflects "pacemaker" activity driven by the high concentrations of HCN4 channels expressed by these cells (Monteggia et al., 2000; Santoro et al., 2000; Gorlich et al., 2013; Oyrer et al., 2019). This pattern of tonic cellular activity contrasts with $\mathrm{LHb}$ neurons, which show much lower levels of baseline activity but a greater propensity to engage in burst firing (Kim and Chang, 2005). Infusion of an HCN antagonist into the $\mathrm{mHb}$ of nicotine-dependent mice precipitated physical signs of withdrawal and increased anxiety-related behavior (Gorlich et al., 2013). No change in the rate of tonic firing of $\mathrm{mHb}$ neurons was detected in mice after chronic nicotine treatment (Gorlich et al., 2013). However, the ability of nicotine to stimulate $\mathrm{mHb}$ neurons was markedly enhanced during nicotine withdrawal in a manner that depended on $\alpha 3 \beta 4^{*}$ nAChRs (Gorlich et al., 2013). Hence, modulatory actions of nicotine on $\mathrm{mHb}$ neurons could alleviate signs of withdrawal regulated by the mHb-IPn circuit and thereby serve as a source of motivation to seek and consume the drug during periods of abstinence, which contributes to relapse vulnerability. Overall, these findings support a major role for the $\mathrm{mHb}-\mathrm{IPn}$ circuit in regulating behavioral abnormalities associated with nicotine withdrawal.

\section{Summary}

The diversity of nAChR subtypes and their dense expression in brain systems involved in reward, aversion, mood regulation, and cognition explain the complex actions of nicotine contained in tobacco products on these behavioral processes. By carefully titrating their nicotine intake to dynamically control the activity of nAChRs in these brain systems, tobacco users can powerfully modulate the motivational, mood, and cognitive processes that drive the initiation, establishment, and maintenance of regular tobacco use. Better understanding of the nAChR subtypes involved in the actions of nicotine in the brain and periphery and identification of the precise circuits, cells, and molecules through which nAChRs transduce responses to nicotine will reveal fundamental new insights into the mechanisms of tobacco dependence. Moreover, because nAChRs have also been implicated in dependence on other classes of abused drugs, including alcohol, opioids, and cocaine, and tobacco smoking is 
highly comorbid with other neuropsychiatric disorders, such as schizophrenia, improved understanding of the mechanisms of tobacco use disorder may provide key insights into other substance use and neuropsychiatric disorders. Ultimately, it is hoped that such information will catalyze the development of "next-generation" therapeutics to combat these disorders.

\section{Acknowledgments}

The authors thank Mrs. Nora McLaughlin for outstanding administrative assistance.

\section{Author Contributions}

Wrote or contributed to the writing of the manuscript: Wills, Ables, Braunscheidel, Caligiuri, Elayouby, Fillinger, Ishikawa, Moen, Kenny.

\section{References}

Ables JL, Görlich A, Antolin-Fontes B, Wang C, Lipford SM, Riad MH, Ren J, Hu F, Luo M, Kenny PJ, et al. (2017) Retrograde inhibition by a specific subset of interpeduncular $\alpha 5$ nicotinic neurons regulates nicotine preference. Proc Natl Acad Sci USA 114:13012-13017.

Acquas E, Carboni E, Leone P, and Di Chiara G (1989) SCH 23390 blocks drugconditioned place-preference and place-aversion: anhedonia (lack of reward) or apathy (lack of motivation) after dopamine-receptor blockade? Psychopharmacology (Berl) 99:151-155

Agetsuma M, Aizawa H, Aoki T, et al. (2010) The habenula is crucial for experiencedependent modification of fear responses in zebrafish. Nat Neurosci 13:1354-1356.

Ahijevych K, Tepper BJ, Graham MC, Holloman C, and Watson WA (2015) Relationships of PROP taste phenotype, taste receptor genotype, and oral nicotine replacement use. Nicotine Tob Res 17:1149-1155.

Ai J, Taylor KM, Lisko JG, Tran H, Watson CH, and Holman MR (2016) Menthol content in US marketed cigarettes. Nicotine Tob Res 18:1575-1580.

Aizawa H, Kobayashi M, Tanaka S, Fukai T, and Okamoto H (2012) Molecular characterization of the subnuclei in rat habenula. J Comp Neurol 520:4051-4066.

Aizawa $\mathrm{H}$ and Zhu M (2019) Toward an understanding of the habenula's various roles in human depression. Psychiatry Clin Neurosci 73:607-612.

Akers AT, Cooper SY, Baumgard ZJ, Casinelli GP, Avelar AJ, and Henderson BJ (2020) Upregulation of nAChRs and changes in excitability on VTA dopamine and GABA neurons correlates to changes in nicotine-reward-related behavior. eNeuro 7:ENEURO.0189-20.2020.

Albuquerque EX, Pereira EF, Alkondon M, and Rogers SW (2009) Mammalian nicotinic acetylcholine receptors: from structure to function. Physiol Rev 89:73-120.

Albuquerque EX, Pereira EF, Castro NG, Alkondon M, Reinhardt S, Schröder H, and Maelicke A (1995) Nicotinic receptor function in the mammalian central nervous system. Ann N Y Acad Sci 757:48-72.

Alderson HL, Latimer MP, and Winn P (2005) Involvement of the laterodorsal tegmental nucleus in the locomotor response to repeated nicotine administration. Neurosci Lett 380:335-339.

Alderson HL, Latimer MP, and Winn P (2006) Intravenous self-administration of nicotine is altered by lesions of the posterior, but not anterior, pedunculopontine tegmental nucleus. Eur J Neurosci 23:2169-2175.

Alderson HL, Latimer MP, and Winn P (2008) A functional dissociation of the anterior and posterior pedunculopontine tegmental nucleus: excitotoxic lesions have differential effects on locomotion and the response to nicotine. Brain Struct Funct 213:247-253.

Alkhlaif Y, Bagdas D, Jackson A, Park AJ, and Damaj IM (2017) Assessment of nicotine withdrawal-induced changes in sucrose preference in mice. Pharmacol Biochem Behav 161:47-52.

Alkondon M and Albuquerque EX (2005) Nicotinic receptor subtypes in rat hippocampal slices are differentially sensitive to desensitization and early in vivo functional up-regulation by nicotine and to block by bupropion. J Pharmacol Exp Ther 313:740-750.

Amos CI, Wu X, Broderick P, Gorlov IP, Gu J, Eisen T, Dong Q, Zhang Q, Gu X, Vijayakrishnan J, et al. (2008) Genome-wide association scan of tag SNPs identifies a susceptibility locus for lung cancer at 15q25.1. Nat Genet 40:616-622.

Andres KH, von Düring M, and Veh RW (1999) Subnuclear organization of the rat habenular complexes. J Comp Neurol 407:130-150.

Antolin-Fontes B, Ables JL, Görlich A, and Ibañez-Tallon I (2015) The habenulointerpeduncular pathway in nicotine aversion and withdrawal. Neuropharmacology 96 (Pt B):213-222.

Antolin-Fontes B, Li K, Ables JL, Riad MH, Görlich A, Williams M, Wang C, Lipford SM, Dao M, Liu J, et al. (2020) The habenular G-protein-coupled receptor 151 regulates synaptic plasticity and nicotine intake. Proc Natl Acad Sci USA 117:5502-5509.

Appleyard SM, Marks D, Kobayashi K, Okano H, Low MJ, and Andresen MC (2007) Visceral afferents directly activate catecholamine neurons in the solitary tract nucleus. J Neurosci 27:13292-13302.
Arvin MC, Jin XT, Yan Y, Wang Y, Ramsey MD, Kim VJ, Beckley NA, Henry BA, and Drenan RM (2019) Chronic nicotine exposure alters the neurophysiology of habenulo-interpeduncular circuitry. $J$ Neurosci 39:4268-4281.

Avale ME, Faure P, Pons S, Robledo P, Deltheil T, David DJ, Gardier AM, Maldonado R, Granon S, Changeux JP, et al. (2008) Interplay of beta2* nicotinic receptors and dopamine pathways in the control of spontaneous locomotion. Proc Natl Acad Sci USA 105:15991-15996.

Avelar AJ, Akers AT, Baumgard ZJ, Cooper SY, Casinelli GP, and Henderson BJ (2019) Why flavored vape products may be attractive: green apple tobacco flavor elicits reward-related behavior, upregulates nAChRs on VTA dopamine neurons, and alters midbrain dopamine and GABA neuron function. Neuropharmacology 158:107729.

Azam L, Maskos U, Changeux JP, Dowell CD, Christensen S, De Biasi M, and McIntosh JM (2010) $\alpha$-Conotoxin BuIA[T5A;P6O]: a novel ligand that discriminates between $\alpha 6 \beta 4$ and $\alpha 6 \beta 2$ nicotinic acetylcholine receptors and blocks nicotine-stimulated norepinephrine release. FASEB J 24:5113-5123.

Azam L, Winzer-Serhan UH, Chen Y, and Leslie FM (2002) Expression of neuronal nicotinic acetylcholine receptor subunit mRNAs within midbrain dopamine neurons. J Comp Neurol 444:260-274.

Azhderian EM, Hefner D, Lin CH, Kaczmarek LK, and Forscher P (1994) Cyclic AMP modulates fast axonal transport in Aplysia bag cell neurons by increasing the probability of single organelle movement. Neuron 12:1223-1233.

Bagdas D, Diester CM, Riley J, Carper M, Alkhlaif Y, AlOmari D, Alayoubi H, Poklis JL, and Damaj MI (2019) Assessing nicotine dependence using an oral nicotine free-choice paradigm in mice. Neuropharmacology 157:107669.

Barlow RB and McLeod LJ (1969) Some studies on cytisine and its methylated derivatives. Br J Pharmacol 35:161-174.

Barrot M, Sesack SR, Georges F, Pistis M, Hong S, and Jhou TC (2012) Braking dopamine systems: a new GABA master structure for mesolimbic and nigrostriatal functions. J Neurosci 32:14094-14101.

Bassareo V, Tanda G, and Di Chiara G (1995) Increase of extracellular dopamine in the medial prefrontal cortex during spontaneous and naloxone-precipitated opiate abstinence. Psychopharmacology (Berl) 122:202-205.

Bell RL, Eiler 2nd BJ, Cook JB, and Rahman S (2009) Nicotinic receptor ligands reduce ethanol intake by high alcohol-drinking HAD-2 rats. Alcohol 43:581-592.

Bello NT and Moran TH (2008) GLP-1 agonists and satiety. Immunol Endocr Metab Agents Med Chem 8:311-316.

Benowitz NL (2009) Pharmacology of nicotine: addiction, smoking-induced disease, and therapeutics. Annu Rev Pharmacol Toxicol 49:57-71.

Berrendero F, Kieffer BL, and Maldonado R (2002) Attenuation of nicotine-induced antinociception, rewarding effects, and dependence in mu-opioid receptor knockout mice. J Neurosci 22:10935-10940.

Berrendero F, Mendizábal V, Robledo P, Galeote L, Bilkei-Gorzo A, Zimmer A, and Maldonado R (2005) Nicotine-induced antinociception, rewarding effects, and physical dependence are decreased in mice lacking the preproenkephalin gene. $J$ Neurosci 25:1103-1112.

Berrettini W, Yuan X, Tozzi F, Song K, Francks C, Chilcoat H, Waterworth D, Muglia P, and Mooser V (2008) Alpha-5/alpha-3 nicotinic receptor subunit alleles increase risk for heavy smoking. Mol Psychiatry 13:368-373.

Besson M, David V, Suarez S, Cormier A, Cazala P, Changeux JP, and Granon S (2006) Genetic dissociation of two behaviors associated with nicotine addiction: beta-2 containing nicotinic receptors are involved in nicotine reinforcement but not in withdrawal syndrome. Psychopharmacology (Berl) 187:189-199.

Bierut LJ, Madden PA, Breslau N, Johnson EO, Hatsukami D, Pomerleau OF Swan GE, Rutter J, Bertelsen S, Fox L, et al. (2007) Novel genes identified in a high-density genome wide association study for nicotine dependence. Hum Mol Genet 16:24-35.

Bierut LJ, Stitzel JA, Wang JC, Hinrichs AL, Grucza RA, Xuei X, Saccone NL, Saccone SF, Bertelsen S, Fox L, et al. (2008) Variants in nicotinic receptors and risk for nicotine dependence. Am J Psychiatry 165:1163-1171.

Blaha CD, Allen LF, Das S, Inglis WL, Latimer MP, Vincent SR, and Winn P (1996) Modulation of dopamine efflux in the nucleus accumbens after cholinergic stimulation of the ventral tegmental area in intact, pedunculopontine tegmental nucleus-lesioned, and laterodorsal tegmental nucleus-lesioned rats. J Neurosci 16:714-722.

Bordia T, Hrachova M, Chin M, McIntosh JM, and Quik M (2012) Varenicline is a potent partial agonist at $\alpha 6 \beta 2^{*}$ nicotinic acetylcholine receptors in rat and monkey striatum. J Pharmacol Exp Ther 342:327-334.

Bozarth MA (1994) Physical dependence produced by central morphine infusions an anatomical mapping study. Neurosci Biobehav Rev 18:373-383.

Breese CR, Marks MJ, Logel J, Adams CE, Sullivan B, Collins AC, and Leonard S (1997) Effect of smoking history on [3H]nicotine binding in human postmortem brain. J Pharmacol Exp Ther 282:7-13.

Brimblecombe KR, Threlfell S, Dautan D, Kosillo P, Mena-Segovia J, and Cragg SJ (2018) Targeted activation of cholinergic interneurons accounts for the modulation of dopamine by striatal nicotinic receptors. eNeuro 5:5.

Broadbent S, Groot-Kormelink PJ, Krashia PA, Harkness PC, Millar NS, Beato M, and Sivilotti LG (2006) Incorporation of the beta3 subunit has a dominantnegative effect on the function of recombinant central-type neuronal nicotinic receptors. Mol Pharmacol 70:1350-1357.

Brody AL, Mandelkern MA, Olmstead RE, Allen-Martinez Z, Scheibal D, Abrams AL, Costello MR, Farahi J, Saxena S, Monterosso J, et al. (2009) Ventral striatal dopamine release in response to smoking a regular vs a denicotinized cigarette. Neuropsychopharmacology 34:282-289.

Brody AL, Mandelkern MA, Olmstead RE, Scheibal D, Hahn E, Shiraga S, ZamoraPaja E, Farahi J, Saxena S, London ED, et al. (2006) Gene variants of brain dopamine pathways and smoking-induced dopamine release in the ventral caudate/nucleus accumbens. Arch Gen Psychiatry 63:808-816.

Brody AL, Mukhin AG, Mamoun MS, Luu T, Neary M, Liang L, Shieh J, Sugar CA, Rose JE, and Mandelkern MA (2014) Brain nicotinic acetylcholine receptor 
availability and response to smoking cessation treatment: a randomized trial. JAMA Psychiatry 71:797-805.

Brody AL, Olmstead RE, London ED, Farahi J, Meyer JH, Grossman P, Lee GS, Huang J, Hahn EL, and Mandelkern MA (2004) Smoking-induced ventral striatum dopamine release. Am J Psychiatry 161:1211-1218.

Bromberg-Martin ES, Matsumoto M, and Hikosaka O (2010) Dopamine in motivational control: rewarding, aversive, and alerting. Neuron 68:815-834

Broms J, Antolin-Fontes B, Tingström A, and Ibañez-Tallon I (2015) Conserved expression of the GPR151 receptor in habenular axonal projections of vertebrates. J Comp Neurol 523:359-380.

Broms J, Grahm M, Haugegaard L, Blom T, Meletis K, and Tingström A (2017) Monosynaptic retrograde tracing of neurons expressing the G-protein coupled receptor Gpr151 in the mouse brain. J Comp Neurol 525:3227-3250.

Brown DA, Docherty RJ, and Halliwell JV (1983) Chemical transmission in the rat interpeduncular nucleus in vitro. $J$ Physiol 341:655-670.

Bruijnzeel AW and Markou A (2004) Adaptations in cholinergic transmission in the ventral tegmental area associated with the affective signs of nicotine withdrawal in rats. Neuropharmacology 47:572-579.

Brunzell DH (2012) Preclinical evidence that activation of mesolimbic alpha 6 subunit containing nicotinic acetylcholine receptors supports nicotine addiction phenotype. Nicotine Tob Res 14:1258-1269.

Brunzell DH, Boschen KE, Hendrick ES, Beardsley PM, and McIntosh JM (2010) Alpha-conotoxin MII-sensitive nicotinic acetylcholine receptors in the nucleus accumbens shell regulate progressive ratio responding maintained by nicotine. Neuropsychopharmacology 35:665-673.

Bryant DL, Free RB, Thomasy SM, Lapinsky DJ, Ismail KA, McKay SB, Bergmeier SC, and McKay DB (2002) Structure-activity studies with ring E analogues of methyllycaconitine on bovine adrenal alpha3beta4* nicotinic receptors. Neurosci Res 42:57-63.

Bueno D, Lima LB, Souza R, Gonçalves L, Leite F, Souza S, Furigo IC, Donato Jr $\mathrm{J}$, and Metzger M (2019) Connections of the laterodorsal tegmental nucleus with the habenular-interpeduncular-raphe system. J Comp Neurol 527:3046-3072.

Cachope R, Mateo Y, Mathur BN, Irving J, Wang HL, Morales M, Lovinger DM, and Cheer JF (2012) Selective activation of cholinergic interneurons enhances accumbal phasic dopamine release: setting the tone for reward processing. Cell Rep 2:33-41.

Caffery PM, Krishnaswamy A, Sanders T, Liu J, Hartlaub H, Klysik J, Cooper E, and Hawrot E (2009) Engineering neuronal nicotinic acetylcholine receptors with functional sensitivity to alpha-bungarotoxin: a novel alpha3-knock-in mouse. Eur $J$ Neurosci 30:2064-2076.

Cahir E, Pillidge K, Drago J, and Lawrence AJ (2011) The necessity of $\alpha 4^{*}$ nicotinic receptors in nicotine-driven behaviors: dissociation between reinforcing and motor effects of nicotine. Neuropsychopharmacology 36:1505-1517.

Cain WS(1980) Sensory attributes of cigarette smoking. In: A Safe Cigarette? (G. B Gori and F. G. Bock eds.), Vol. 3. Cold Spring Harbor Laboratory, New York, Banbury Center.

Campling BG, Kuryatov A, and Lindstrom J (2013) Acute activation, desensitization and smoldering activation of human acetylcholine receptors. PLoS One 8: 79653

Carboni E, Bortone L, Giua C, and Di Chiara G (2000a) Dissociation of physical abstinence signs from changes in extracellular dopamine in the nucleus accumbens and in the prefrontal cortex of nicotine dependent rats. Drug Alcohol Depend 58:93-102.

Carboni E, Silvagni A, Rolando MT, and Di Chiara G (2000b) Stimulation of in vivo dopamine transmission in the bed nucleus of stria terminalis by reinforcing drugs. J Neurosci 20:RC102.

Champtiaux N, Gotti C, Cordero-Erausquin M, David DJ, Przybylski C, Léna C, Clementi F, Moretti M, Rossi FM, Le Novère N, et al. (2003) Subunit composition of functional nicotinic receptors in dopaminergic neurons investigated with knock-out mice. J Neurosci 23:7820-7829.

Champtiaux N, Han ZY, Bessis A, Rossi FM, Zoli M, Marubio L, McIntosh JM, and Changeux JP (2002) Distribution and pharmacology of alpha 6-containing nicotinic acetylcholine receptors analyzed with mutant mice. $J$ Neurosci 22:1208-1217.

Chang FC and Scott TR (1984) Conditioned taste aversions modify neural responses in the rat nucleus tractus solitarius. J Neurosci 4:1850-1862.

Changeux JP (1979) The acetylcholine receptor: an "allosteric" membrane protein. Harvey Lect 75:85-254.

Changeux JP, Devillers-Thiéry A, and Chemouilli P (1984) Acetylcholine receptor: an allosteric protein. Science 225:1335-1345.

Changeux JP and Taly A (2008) Nicotinic receptors, allosteric proteins and medicine. Trends Mol Med 14:93-102.

Charpantier E, Barnéoud P, Moser P, Besnard F, and Sgard F (1998) Nicotinic acetylcholine subunit mRNA expression in dopaminergic neurons of the rat substantia nigra and ventral tegmental area. Neuroreport 9:3097-3101.

Chatterjee S, Santos N, Holgate J, Haass-Koffler CL, Hopf FW, Kharazia V, Lester $\mathrm{H}$, Bonci A, and Bartlett SE (2013) The $\alpha 5$ subunit regulates the expression and function of $\alpha 4^{*}$-containing neuronal nicotinic acetylcholine receptors in the ventral-tegmental area. PLoS One 8:e68300.

Chen C and Regehr WG (1997) The mechanism of cAMP-mediated enhancement at a cerebellar synapse. $J$ Neurosci 17:8687-8694.

Chen L and Lodge DJ (2013) The lateral mesopontine tegmentum regulates both tonic and phasic activity of VTA dopamine neurons. $J$ Neurophysiol 110:2287-2294

Chen T, Dong YX, and Li YQ (2003) Fos expression in serotonergic neurons in the rat brainstem following noxious stimuli: an immunohistochemical doublelabelling study. J Anat 203:579-588.

Chiang YT, Ip W, and Jin T (2012) The role of the Wnt signaling pathway in incretin hormone production and function. Front Physiol 3:273.

Cimino M, Marini P, Fornasari D, Cattabeni F, and Clementi F (1992) Distribution of nicotinic receptors in cynomolgus monkey brain and ganglia: localization of alpha 3 subunit mRNA, alpha-bungarotoxin and nicotine binding sites. Neuroscience 51:77-86.

Clarke PB, Fu DS, Jakubovic A, and Fibiger HC (1988) Evidence that mesolimbic dopaminergic activation underlies the locomotor stimulant action of nicotine in rats. J Pharmacol Exp Ther 246:701-708.

Clarke PB, Hamill GS, Nadi NS, Jacobowitz DM, and Pert A (1986) 3H-nicotineand 125I-alpha-bungarotoxin-labeled nicotinic receptors in the interpeduncular nucleus of rats. II. Effects of habenular deafferentation. J Comp Neurol 251:407-413.

Clarke PB and Kumar R (1983a) Characterization of the locomotor stimulant action of nicotine in tolerant rats. Br J Pharmacol 80:587-594

Clarke PB and Kumar R (1983b) The effects of nicotine on locomotor activity in non-tolerant and tolerant rats. Br J Pharmacol 78:329-337.

Clarke PB and Pert A (1985) Autoradiographic evidence for nicotine receptors on nigrostriatal and mesolimbic dopaminergic neurons. Brain Res 348:355-358.

Clarke PB, Schwartz RD, Paul SM, Pert CB, and Pert A (1985) Nicotinic binding in rat brain: autoradiographic comparison of $[3 \mathrm{H}]$ acetylcholine, $[3 \mathrm{H}]$ nicotine, and [125I]-alpha-bungarotoxin. J Neurosci 5:1307-1315.

Coe JW, Brooks PR, Vetelino MG, Wirtz MC, Arnold EP, Huang J, Sands SB, Davis TI, Lebel LA, Fox CB, et al. (2005) Varenicline: an alpha4beta2 nicotinic receptor partial agonist for smoking cessation. J Med Chem 48:3474-3477.

Cohen C, Bergis OE, Galli F, Lochead AW, Jegham S, Biton B, Leonardon J, Avenet P, Sgard F, Besnard F, et al. (2003) SSR591813, a novel selective and partial alpha4beta2 nicotinic receptor agonist with potential as an aid to smoking cessation. J Pharmacol Exp Ther 306:407-420.

Coimbra B, Soares-Cunha C, Vasconcelos NAP, Domingues AV, Borges S, Sousa N, and Rodrigues AJ (2019) Role of laterodorsal tegmentum projections to nucleus accumbens in reward-related behaviors. Nat Commun 10:4138.

Collaborators GBDT; GBD 2015 Tobacco Collaborators (2017) Smoking prevalence and attributable disease burden in 195 countries and territories, 1990-2015: a systematic analysis from the Global Burden of Disease Study 2015. Lancet 389:1885-1906.

Connolly JG, Gibb AJ, and Colquhoun D (1995) Heterogeneity of neuronal nicotinic acetylcholine receptors in thin slices of rat medial habenula. J Physiol 484:87-105.

Contestabile A, Villani L, Fasolo A, Franzoni MF, Gribaudo L, Oktedalen O, and Fonnum F (1987) Topography of cholinergic and substance P pathways in the habenulo-interpeduncular system of the rat. An immunocytochemical and microchemical approach. Neuroscience 21:253-270.

Correa VL, Flores RJ, Carcoba LM, Arreguin MC, and O'Dell LE (2019) Sex differences in cholinergic systems in the interpeduncular nucleus following nicotine exposure and withdrawal. Neuropharmacology 158:107714.

Corrigall WA and Coen KM (1989) Nicotine maintains robust self-administration in rats on a limited-access schedule. Psychopharmacology (Berl) 99:473-478.

Corrigall WA and Coen KM (1991) Selective dopamine antagonists reduce nicotine self-administration. Psychopharmacology (Berl) 104:171-176.

Corrigall WA, Coen KM, Zhang J, and Adamson L (2002) Pharmacological manipulations of the pedunculopontine tegmental nucleus in the rat reduce selfadministration of both nicotine and cocaine. Psychopharmacology (Berl) 160:198-205

Corrigall WA, Franklin KB, Coen KM, and Clarke PB (1992) The mesolimbic dopaminergic system is implicated in the reinforcing effects of nicotine. Psychopharmacology (Berl) 107:285-289.

Cover, K. K., Gyawali, U., Kerkhoff, W. G., et al. (2019) Activation of the rostral intralaminar thalamus drives reinforcement through striatal dopamine release. Cell Rep 26, 13891398 e1383.

Cragg SJ (2003) Variable dopamine release probability and short-term plasticity between functional domains of the primate striatum. J Neurosci 23:4378-4385.

Cui C, Booker TK, Allen RS, Grady SR, Whiteaker P, Marks MJ, Salminen O, Tritto T, Butt CM, Allen WR, et al. (2003) The beta3 nicotinic receptor subunit: a component of alpha-conotoxin MII-binding nicotinic acetylcholine receptors that modulate dopamine release and related behaviors. J Neurosci 23:11045-11053.

D'Silva J, Cohn AM, Johnson AL, and Villanti AC (2018) Differences in subjective experiences to first use of menthol and nonmenthol cigarettes in a national sample of young adult cigarette smokers. Nicotine Tob Res 20:1062-1068.

Dahl M, Erickson RP, and Simon SA (1997) Neural responses to bitter compounds in rats. Brain Res $\mathbf{7 5 6}: 22-34$

Dao DQ, Perez EE, Teng Y, Dani JA, and De Biasi M (2014) Nicotine enhances excitability of medial habenular neurons via facilitation of neurokinin signaling. $J$ Neurosci 34:4273-4284.

Dash B, Bhakta M, Chang Y, and Lukas RJ (2012) Modulation of recombinant, $\alpha 2^{*}$, $\alpha 3^{*}$ or $\alpha 4^{*}$-nicotinic acetylcholine receptor $(\mathrm{nAChR})$ function by $\mathrm{nAChR} \beta 3$ subunits. $J$ Neurochem 121:349-361.

Dash B and Li MD (2014) Two rare variations, D478N and D478E, that occur at the same amino acid residue in nicotinic acetylcholine receptor (nAChR) $\alpha 2$ subunit influence $\mathrm{nAChR}$ function. Neuropharmacology 85:471-481.

Dautan D, Huerta-Ocampo I, Witten IB, Deisseroth K, Bolam JP, Gerdjikov T, and Mena-Segovia J (2014) A major external source of cholinergic innervation of the striatum and nucleus accumbens originates in the brainstem. $J$ Neurosci 34:4509-4518.

Dautan D, Souza AS, Huerta-Ocampo I, Valencia M, Assous M, Witten IB Deisseroth K, Tepper JM, Bolam JP, Gerdjikov TV, et al. (2016) Segregated cholinergic transmission modulates dopamine neurons integrated in distinct functional circuits. Nat Neurosci 19:1025-1033.

David V, Besson M, Changeux JP, Granon S, and Cazala P (2006) Reinforcing effects of nicotine microinjections into the ventral tegmental area of mice: dependence on cholinergic nicotinic and dopaminergic D1 receptors. Neuropharmacology 50:1030-1040.

David V, Segu L, Buhot MC, Ichaye M, and Cazala P (2004) Rewarding effects elicited by cocaine microinjections into the ventral tegmental area of $\mathrm{C} 57 \mathrm{BL} / 6 \mathrm{mice}$ involvement of dopamine D1 and serotonin1B receptors. Psychopharmacology (Berl) 174:367-375. 
Dawe S, Gerada C, Russell MA, and Gray JA (1995) Nicotine intake in smokers increases following a single dose of haloperidol. Psychopharmacology (Berl) 117:110-115.

De Biasi M and Dani JA (2011) Reward, addiction, withdrawal to nicotine. Annu Rev Neurosci 34:105-130.

De Biasi M and Salas R (2008) Influence of neuronal nicotinic receptors over nicotine addiction and withdrawal. Exp Biol Med (Maywood) 233:917-929.

de Wit H and Phillips TJ (2012) Do initial responses to drugs predict future use or abuse? Neurosci Biobehav Rev 36:1565-1576.

DeGroot SR, Zhao-Shea R, Chung L, Klenowski PM, Sun F, Molas S, Gardner PD, Li Y, and Tapper AR (2020) Midbrain dopamine controls anxiety-like behavior by engaging unique interpeduncular nucleus microcircuitry. Biol Psychiatry 88:855-866.

Dehkordi O, Rose JE, Asadi S, Manaye KF, Millis RM, and Jayam-Trouth A (2015) Neuroanatomical circuitry mediating the sensory impact of nicotine in the central nervous system. J Neurosci Res 93:230-243.

Dehkordi O, Rose JE, Millis RM, Dalivand MM, and Johnson SM (2018) GABAergic neurons as putative neurochemical substrate mediating aversive effects of nicotine. J Alcohol Drug Depend 6:6.

Demontis D, Rajagopal VM, Thorgeirsson TE, Als TD, Grove J, Leppälä K, Gudbjartsson DF, Pallesen J, Hjorthøj C, Reginsson GW, et al. (2019) Genomewide association study implicates CHRNA2 in cannabis use disorder. Nat Neurosci 22:1066-1074

Deneris ES, Connolly J, Rogers SW, and Duvoisin R (1991) Pharmacological and functional diversity of neuronal nicotinic acetylcholine receptors. Trends Pharmacol Sci 12:34-40.

DeNoble VJ and Mele PC (2006) Intravenous nicotine self-administration in rats: effects of mecamylamine, hexamethonium and naloxone. Psychopharmacology (Berl) 184:266-272.

Di Chiara G (2000) Role of dopamine in the behavioural actions of nicotine related to addiction. Eur J Pharmacol 393:295-314.

Di Chiara G and Imperato A (1988) Drugs abused by humans preferentially increase synaptic dopamine concentrations in the mesolimbic system of freely moving rats. Proc Natl Acad Sci USA 85:5274-5278.

Doherty K, Kinnunen T, Militello FS, and Garvey AJ (1995) Urges to smoke during the first month of abstinence: relationship to relapse and predictors. Psychopharmacology (Berl) 119:171-178.

Doll R, Peto R, Boreham J, and Sutherland I (2004) Mortality in relation to smoking: 50 years' observations on male British doctors. BMJ 328:1519.

Doll R, Peto R, Wheatley K, Gray R, and Sutherland I (1994) Mortality in relation to smoking: 40 years' observations on male British doctors. BMJ 309:901-911.

Donovick PJ, Burright RG, and Zuromski E (1970) Localization of quinine aversion within the septum, habenula, and interpeduncular nucleus of the rat. J Comp Physiol Psychol 71:376-383.

Drenan RM, Grady SR, Steele AD, McKinney S, Patzlaff NE, McIntosh JM, Marks MJ, Miwa JM, and Lester HA (2010) Cholinergic modulation of locomotion and striatal dopamine release is mediated by alpha6alpha4* nicotinic acetylcholine receptors. J Neurosci 30:9877-9889.

Drenan RM, Nashmi R, Imoukhuede P, Just H, McKinney S, and Lester HA (2008) Subcellular trafficking, pentameric assembly, and subunit stoichiometry of neuronal nicotinic acetylcholine receptors containing fluorescently labeled alpha6 and beta3 subunits. Mol Pharmacol 73:27-41.

Duncan A, Heyer MP, Ishikawa M, Caligiuri SPB, Liu XA, Chen Z, Micioni Di Bonaventura MV, Elayouby KS, Ables JL, Howe WM, et al. (2019) Habenular TCF7L2 links nicotine addiction to diabetes. Nature 574:372-377.

Durand-de Cuttoli R, Mondoloni S, Marti F, Lemoine D, Nguyen C, Naudé J, d'Izarny-Gargas T, Pons S, Maskos U, Trauner D, et al. (2018) Manipulating midbrain dopamine neurons and reward-related behaviors with light-controllable nicotinic acetylcholine receptors. eLife 7:7.

Dwoskin LP, Smith AM, Wooters TE, Zhang Z, Crooks PA, and Bardo MT (2009) Nicotinic receptor-based therapeutics and candidates for smoking cessation. Biochem Pharmacol 78:732-743.

Edwards FA, Gibb AJ, and Colquhoun D (1992) ATP receptor-mediated synaptic currents in the central nervous system. Nature 359:144-147.

Elayouby KS, Ishikawa M, Dukes AJ, Smith ACW, Lu Q, Fowler CD, and Kenny PJ (2021) $\alpha 3^{*}$ Nicotinic acetylcholine receptors in the habenula-interpeduncular nucleus circuit regulate nicotine intake. $J$ Neurosci 41:1779-1787.

Elgoyhen AB, Johnson DS, Boulter J, Vetter DE, and Heinemann S (1994) Alpha 9: an acetylcholine receptor with novel pharmacological properties expressed in rat cochlear hair cells. Cell 79:705-715

Elgoyhen AB, Vetter DE, Katz E, Rothlin CV, Heinemann SF, and Boulter J (2001) alpha10: a determinant of nicotinic cholinergic receptor function in mammalian vestibular and cochlear mechanosensory hair cells. Proc Natl Acad Sci USA 98:3501-3506

Engle SE, Shih PY, McIntosh JM, and Drenan RM (2013) $\alpha 4 \alpha 6 \beta 2 *$ nicotinic acetylcholine receptor activation on ventral tegmental area dopamine neurons is sufficient to stimulate a depolarizing conductance and enhance surface AMPA receptor function. Mol Pharmacol 84:393-406.

Epping-Jordan MP, Watkins SS, Koob GF, and Markou A (1998) Dramatic decreases in brain reward function during nicotine withdrawal. Nature 393:76-79.

Espejo EF, Serrano MI, Caillé S, and Stinus L (2001) Behavioral expression of opiate withdrawal is altered after prefrontocortical dopamine depletion in rats: monoaminergic correlates. Neuropsychopharmacology 25:204-212.

Esterlis I, Hillmer AT, Bois F, Pittman B, McGovern E, O'Malley SS, Picciotto MR Yang BZ, Gelernter J, and Cosgrove KP (2016) CHRNA4 and ANKK1 polymorphisms influence smoking-induced nicotinic acetylcholine receptor upregulation. Nicotine Tob Res 18:1845-1852.

Esterlis I, Ranganathan M, Bois F, Pittman B, Picciotto MR, Shearer L, Anticevic A, Carlson J, Niciu MJ, Cosgrove KP, et al. (2014) In vivo evidence for $\beta 2$ nicotinic acetylcholine receptor subunit upregulation in smokers as compared with nonsmokers with schizophrenia. Biol Psychiatry 76:495-502.
Etter JF (2006) Cytisine for smoking cessation: a literature review and a metaanalysis. Arch Intern Med 166:1553-1559.

Exley R, Clements MA, Hartung H, McIntosh JM, and Cragg SJ (2008) Alpha6containing nicotinic acetylcholine receptors dominate the nicotine control of dopamine neurotransmission in nucleus accumbens. Neuropsychopharmacology 33:2158-2166.

Exley R and Cragg SJ (2008) Presynaptic nicotinic receptors: a dynamic and diverse cholinergic filter of striatal dopamine neurotransmission. $\mathrm{Br} J$ Pharmacol 153 (Suppl 1):S283-S297.

Exley R, Maubourguet N, David V, Eddine R, Evrard A, Pons S, Marti F, Threlfell S, Cazala P, McIntosh JM, et al. (2011) Distinct contributions of nicotinic acetylcholine receptor subunit alpha4 and subunit alpha6 to the reinforcing effects of nicotine. Proc Natl Acad Sci USA 108:7577-7582.

Exley R, McIntosh JM, Marks MJ, Maskos U, and Cragg SJ (2012) Striatal $\alpha 5$ nicotinic receptor subunit regulates dopamine transmission in dorsal striatum. $J$ Neurosci 32:2352-2356.

Ezzati M and Lopez AD (2003) Estimates of global mortality attributable to smoking in 2000. Lancet 362:847-852.

Fagerström KO (1988) Efficacy of nicotine chewing gum: a review. Prog Clin Biol Res 261:109-128.

Fagerström KO, Schneider NG, and Lunell E (1993) Effectiveness of nicotine patch and nicotine gum as individual versus combined treatments for tobacco withdrawal symptoms. Psychopharmacology (Berl) 111:271-277.

Faget L, Osakada F, Duan J, Ressler R, Johnson AB, Proudfoot JA, Yoo JH, Callaway EM, and Hnasko TS (2016) Afferent inputs to neurotransmitterdefined cell types in the ventral tegmental area. Cell Rep 15:2796-2808.

Feyerabend C, Higenbottam T, and Russell MA (1982) Nicotine concentrations in urine and saliva of smokers and non-smokers. Br Med J (Clin Res Ed) 284:1002-1004.

File SE, Cheeta S, and Kenny PJ (2000) Neurobiological mechanisms by which nicotine mediates different types of anxiety. Eur J Pharmacol 393:231-236.

File SE, Kenny PJ, and Ouagazzal AM (1998) Bimodal modulation by nicotine of anxiety in the social interaction test: role of the dorsal hippocampus. Behav Neurosci 112:1423-1429.

Flannery JS, Riedel MC, Poudel R, Laird AR, Ross TJ, Salmeron BJ, Stein EA, and Sutherland MT (2019) Habenular and striatal activity during performance feedback are differentially linked with state-like and trait-like aspects of tobacco use disorder. Sci Adv 5:eaax2084.

Flores CM, Rogers SW, Pabreza LA, Wolfe BB, and Kellar KJ (1992) A subtype of nicotinic cholinergic receptor in rat brain is composed of alpha 4 and beta 2 subunits and is up-regulated by chronic nicotine treatment. Mol Pharmacol 41:31-37.

Fonck C, Cohen BN, Nashmi R, Whiteaker P, Wagenaar DA, Rodrigues-Pinguet N, Deshpande P, McKinney S, Kwoh S, Munoz J, et al. (2005) Novel seizure phenotype and sleep disruptions in knock-in mice with hypersensitive alpha $4^{*}$ nicotinic receptors. $J$ Neurosci 25:11396-11411.

Fonck C, Nashmi R, Salas R, Zhou C, Huang Q, De Biasi M, Lester RA, and Lester HA (2009) Demonstration of functional alpha4-containing nicotinic receptors in the medial habenula. Neuropharmacology 56:247-253.

Forget B, Scholze P, Langa F, Morel C, Pons S, Mondoloni S, Besson M, Durand-de Cuttoli R, Hay A, Tricoire L, et al. (2018) A human polymorphism in CHRNA5 is linked to relapse to nicotine seeking in transgenic rats. Curr Biol 28:3244-3253.e7.

Forsayeth JR and Kobrin E (1997) Formation of oligomers containing the beta 3 and beta4 subunits of the rat nicotinic receptor. $J$ Neurosci 17:1531-1538.

Forster GL and Blaha CD (2000) Laterodorsal tegmental stimulation elicits dopamine efflux in the rat nucleus accumbens by activation of acetylcholine and glutamate receptors in the ventral tegmental area. Eur J Neurosci 12:3596-3604.

Fowler CD, Arends MA, and Kenny PJ (2008) Subtypes of nicotinic acetylcholine receptors in nicotine reward, dependence, and withdrawal: evidence from genetically modified mice. Behav Pharmacol 19:461-484.

Fowler CD and Kenny PJ (2011) Intravenous nicotine self-administration and cueinduced reinstatement in mice: effects of nicotine dose, rate of drug infusion and prior instrumental training. Neuropharmacology 61:687-698.

Fowler, C. D. and Kenny, P. J. (2014) Nicotine aversion: Neurobiological mechanisms and relevance to tobacco dependence vulnerability. Neuropharmacology 76 Pt B, 533-544.

Fowler CD, Lu Q, Johnson PM, Marks MJ, and Kenny PJ (2011) Habenular $\alpha 5$ nicotinic receptor subunit signalling controls nicotine intake. Nature 471:597-601.

Fowler, C. D., Tuesta, L. and Kenny, P. J. (2013) Role of alpha5* nicotinic acetylcholine receptors in the effects of acute and chronic nicotine treatment on brain reward function in mice. Psychopharmacology (Berl).

Frahm S, Antolin-Fontes B, Görlich A, Zander JF, Ahnert-Hilger G, and Ibañez Tallon I (2015) An essential role of acetylcholine-glutamate synergy at habenular synapses in nicotine dependence. eLife 4:e11396.

Frahm S, Slimak MA, Ferrarese L, Santos-Torres J, Antolin-Fontes B, Auer S, Filkin S, Pons S, Fontaine JF, Tsetlin V, et al. (2011) Aversion to nicotine is regulated by the balanced activity of $\beta 4$ and $\alpha 5$ nicotinic receptor subunits in the medial habenula. Neuron 70:522-535.

Fu Y, Matta SG, Gao W, Brower VG, and Sharp BM (2000) Systemic nicotine stimulates dopamine release in nucleus accumbens: re-evaluation of the role of $\mathrm{N}$-methyl-D-aspartate receptors in the ventral tegmental area. J Pharmacol Exp Ther 294:458-465.

Fu Y, Matta SG, Kane VB, and Sharp BM (2003) Norepinephrine release in amygdala of rats during chronic nicotine self-administration: an in vivo microdialysis study. Neuropharmacology 45:514-523.

Fucile S, Barabino B, Palma E, Grassi F, Limatola C, Mileo AM, Alemà S, Ballivet M, and Eusebi F (1997) Alpha 5 subunit forms functional alpha 3 beta 4 alpha 5 nAChRs in transfected human cells. Neuroreport 8:2433-2436.

Galeote L, Berrendero F, Bura SA, Zimmer A, and Maldonado R (2009) Prodynorphin gene disruption increases the sensitivity to nicotine self-administration in mice. Int J Neuropsychopharmacol 12:615-625. 
Gallego X, Cox RJ, Laughlin JR, Stitzel JA, and Ehringer MA (2013) Alternative CHRNB4 3'-UTRs mediate the allelic effects of SNP rs1948 on gene expression. PLoS One 8:e63699.

Garcia-Diaz DE, Jimenez-Montufar LL, Guevara-Aguilar R, Wayner MJ, and Armstrong DL (1988) Olfactory and visceral projections to the nucleus of the solitary tract. Physiol Behav 44:619-624.

Geng F, Liu JY, Chen XW, Zou WJ, Wu JL, Rodriguez G, Peng C, Tian J, and Lu GF (2019) ErbB4 receptors in the medial habenula regulate contextual fear memory. Pharmacology 103:68-75.

Gerzanich V, Wang F, Kuryatov A, and Lindstrom J (1998) alpha 5 Subunit alters desensitization, pharmacology, $\mathrm{Ca}++$ permeability and $\mathrm{Ca}++$ modulation of human neuronal alpha 3 nicotinic receptors. J Pharmacol Exp Ther 286:311-320.

Giniatullin R, Nistri A, and Yakel JL (2005) Desensitization of nicotinic ACh receptors: shaping cholinergic signaling. Trends Neurosci 28:371-378.

Girod R, Barazangi N, McGehee D, and Role LW (2000) Facilitation of glutamatergic neurotransmission by presynaptic nicotinic acetylcholine receptors. Neuropharmacology 39:2715-2725.

Glick SD, Sell EM, McCallum SE, and Maisonneuve IM (2011) Brain regions mediating $\alpha 3 \beta 4$ nicotinic antagonist effects of $18-\mathrm{MC}$ on nicotine self-administration. Eur J Pharmacol 669:71-75.

Göke R, Larsen PJ, Mikkelsen JD, and Sheikh SP (1995) Distribution of GLP-1 binding sites in the rat brain: evidence that exendin- 4 is a ligand of brain GLP-1 binding sites. Eur J Neurosci 7:2294-2300.

Goldberg SR and Spealman RD (1982) Maintenance and suppression of behavior by intravenous nicotine injections in squirrel monkeys. Fed Proc 41:216-220.

Goldberg SR and Spealman RD (1983) Suppression of behavior by intravenous injections of nicotine or by electric shocks in squirrel monkeys: effects of chlordiazepoxide and mecamylamine. $J$ Pharmacol Exp Ther 224:334-340.

Goldberg SR, Spealman RD, and Goldberg DM (1981) Persistent behavior at high rates maintained by intravenous self-administration of nicotine. Science 214:573-575.

Good CH and Lupica CR (2009) Properties of distinct ventral tegmental area synapses activated via pedunculopontine or ventral tegmental area stimulation in vitro. J Physiol 587:1233-1247.

Görlich A, Antolin-Fontes B, Ables JL, Frahm S, Slimak MA, Dougherty JD, and Ibañez-Tallon I (2013) Reexposure to nicotine during withdrawal increases the pacemaking activity of cholinergic habenular neurons. Proc Natl Acad Sci USA 110:17077-17082.

Goto M, Swanson LW, and Canteras NS (2001) Connections of the nucleus incertus. J Comp Neurol 438:86-122.

Gotti C, Clementi F, Fornari A, Gaimarri A, Guiducci S, Manfredi I, Moretti M, Pedrazzi P, Pucci L, and Zoli M (2009) Structural and functional diversity of native brain neuronal nicotinic receptors. Biochem Pharmacol 78:703-711.

Gotti C, Guiducci S, Tedesco V, Corbioli S, Zanetti L, Moretti M, Zanardi A, Rimondini R, Mugnaini M, Clementi F, et al. (2010) Nicotinic acetylcholine receptors in the mesolimbic pathway: primary role of ventral tegmental area alpha6beta2* receptors in mediating systemic nicotine effects on dopamine release, locomotion, and reinforcement. $J$ Neurosci 30:5311-5325.

Gotti C, Moretti M, Clementi F, Riganti L, McIntosh JM, Collins AC, Marks MJ, and Whiteaker P (2005) Expression of nigrostriatal alpha 6-containing nicotinic acetylcholine receptors is selectively reduced, but not eliminated, by beta 3 subunit gene deletion. Mol Pharmacol 67:2007-2015

Gotti C, Moretti M, Gaimarri A, Zanardi A, Clementi F, and Zoli M (2007) Heterogeneity and complexity of native brain nicotinic receptors. Biochem Pharmacol 74:1102-1111.

Gotti C, Moretti M, Meinerz NM, Clementi F, Gaimarri A, Collins AC, and Marks MJ (2008) Partial deletion of the nicotinic cholinergic receptor alpha 4 or beta 2 subunit genes changes the acetylcholine sensitivity of receptor-mediated $86 \mathrm{Rb}+$ efflux in cortex and thalamus and alters relative expression of alpha 4 and beta 2 subunits. Mol Pharmacol 73:1796-1807.

Gotti C, Riganti L, Vailati S, and Clementi F (2006a) Brain neuronal nicotinic receptors as new targets for drug discovery. Curr Pharm Des 12:407-428.

Gotti C, Zoli M, and Clementi F (2006b) Brain nicotinic acetylcholine receptors: native subtypes and their relevance. Trends Pharmacol Sci 27:482-491.

Goutier W, Lowry JP, McCreary AC, and O'Connor JJ (2016) Frequency-dependent modulation of dopamine release by nicotine and dopamine D1 receptor ligands: an in vitro fast cyclic voltammetry study in rat striatum. Neurochem Res 41:945-950.

Grady SR, Meinerz NM, Cao J, Reynolds AM, Picciotto MR, Changeux JP, McIntosh JM, Marks MJ, and Collins AC (2001) Nicotinic agonists stimulate acetylcholine release from mouse interpeduncular nucleus: a function mediated by a different nAChR than dopamine release from striatum. $J$ Neurochem 76:258-268.

Grady SR, Moretti M, Zoli M, Marks MJ, Zanardi A, Pucci L, Clementi F, and Gotti C (2009) Rodent habenulo-interpeduncular pathway expresses a large variety of uncommon nAChR subtypes, but only the alpha3beta4* and alpha3beta3beta4* subtypes mediate acetylcholine release. J Neurosci 29:2272-2282.

Grady SR, Salminen O, Laverty DC, Whiteaker P, McIntosh JM, Collins AC, and Marks MJ (2007) The subtypes of nicotinic acetylcholine receptors on dopaminergic terminals of mouse striatum. Biochem Pharmacol 74:1235-1246.

Grady SR, Salminen O, McIntosh JM, Marks MJ, and Collins AC (2010) Mouse striatal dopamine nerve terminals express alpha4alpha5beta2 and two stoichiometric forms of alpha4beta $2 *$-nicotinic acetylcholine receptors. $J \mathrm{Mol}$ Neurosci 40:91-95.

Grady SR, Wageman CR, Patzlaff NE, and Marks MJ (2012) Low concentrations of nicotine differentially desensitize nicotinic acetylcholine receptors that include $\alpha 5$ or $\alpha 6$ subunits and that mediate synaptosomal neurotransmitter release. Neuropharmacology 62:1935-1943.

Gray MA and Critchley HD (2007) Interoceptive basis to craving. Neuron 54:183-186.

Grenhoff J, Aston-Jones G, and Svensson TH (1986) Nicotinic effects on the firing pattern of midbrain dopamine neurons. Acta Physiol Scand 128:351-358.
Grieder TE, Besson M, Maal-Bared G, Pons S, Maskos U, and van der Kooy D (2019) $\beta 2 *$ nAChRs on VTA dopamine and GABA neurons separately mediate nicotine aversion and reward. Proc Natl Acad Sci USA 116:25968-25973.

Grieder TE, George O, Tan H, George SR, Le Foll B, Laviolette SR, and van der Kooy D (2012) Phasic D1 and tonic D2 dopamine receptor signaling double dissociate the motivational effects of acute nicotine and chronic nicotine withdrawal. Proc Natl Acad Sci USA 109:3101-3106.

Grieder TE, George O, Yee M, Bergamini MA, Chwalek M, Maal-Bared G, VargasPerez H, and van der Kooy D (2017) Deletion of $\alpha 5$ nicotine receptor subunits abolishes nicotinic aversive motivational effects in a manner that phenocopies dopamine receptor antagonism. Eur J Neurosci 46:1673-1681.

Grieder TE, Herman MA, Contet C, Tan LA, Vargas-Perez H, Cohen A, Chwalek M, Maal-Bared G, Freiling J, Schlosburg JE, et al. (2014) VTA CRF neurons mediate the aversive effects of nicotine withdrawal and promote intake escalation. Nat Neurosci 17:1751-1758.

Grillner P and Svensson TH (2000) Nicotine-induced excitation of midbrain dopamine neurons in vitro involves ionotropic glutamate receptor activation. Synapse 38:1-9.

Groenewegen HJ and Steinbusch HW (1984) Serotonergic and non-serotonergic projections from the interpeduncular nucleus to the ventral hippocampus in the rat. Neurosci Lett 51:19-24.

Grucza RA, Wang JC, Stitzel JA, Hinrichs AL, Saccone SF, Saccone NL, Bucholz KK, Cloninger CR, Neuman RJ, Budde JP, et al. (2008) A risk allele for nicotine dependence in CHRNA5 is a protective allele for cocaine dependence. Biol Psychiatry 64:922-929.

Gu S, Matta JA, Davini WB, Dawe GB, Lord B, and Bredt DS (2019) $\alpha$ 6-Containing nicotinic acetylcholine receptor reconstitution involves mechanistically distinct accessory components. Cell Rep 26:866-874.e3.

Guo X and Lester RA (2007) Ca2+ flux and signaling implications by nicotinic acetylcholine receptors in rat medial habenula. J Neurophysiol 97:83-92.

Gutzwiller JP, Göke B, Drewe J, Hildebrand P, Ketterer S, Handschin D, Winterhalder R, Conen D, and Beglinger C (1999) Glucagon-like peptide-1: a potent regulator of food intake in humans. Gut 44:81-86.

Harmey D, Griffin PR, and Kenny PJ (2012) Development of novel pharmacotherapeutics for tobacco dependence: progress and future directions. Nicotine Tob Res 14:1300-1318.

Harpsoe K, Ahring PK, Christensen JK, Jensen ML, Peters D, and Balle T (2011) Unraveling the high- and low-sensitivity agonist responses of nicotinic acetylcholine receptors. $J$ Neurosci 31:10759-10766.

Harrington L, Viñals X, Herrera-Solís A, Flores A, Morel C, Tolu S, Faure P, Maldonado R, Maskos U, and Robledo P (2016) Role of $\beta 4^{*}$ nicotinic acetylcholine receptors in the habenulo-interpeduncular pathway in nicotine reinforcement in mice. Neuropsychopharmacology 41:1790-1802.

Harris AC, Muelken P, Swain Y, Palumbo M, Jain V, Goniewicz ML, Stepanov I, and LeSage MG (2019) Non-nicotine constituents in e-cigarette aerosol extract attenuate nicotine's aversive effects in adolescent rats. Drug Alcohol Depend 203:51-60

Harrison AA, Gasparini F, and Markou A (2002) Nicotine potentiation of brain stimulation reward reversed by $\mathrm{DH} \beta \mathrm{E}$ and $\mathrm{SCH} 23390$, but not by eticlopride, LY 314582 or MPEP in rats. Psychopharmacology (Berl) 160:56-66.

Harvey DM, Yasar S, Heishman SJ, Panlilio LV, Henningfield JE, and Goldberg SR (2004) Nicotine serves as an effective reinforcer of intravenous drug-taking behavior in human cigarette smokers. Psychopharmacology (Berl) 175:134-142.

Harvey SC and Luetje CW (1996) Determinants of competitive antagonist sensitivity on neuronal nicotinic receptor beta subunits. J Neurosci 16:3798-3806.

Harvey SC, Maddox FN, and Luetje CW (1996) Multiple determinants of dihydrobeta-erythroidine sensitivity on rat neuronal nicotinic receptor alpha subunits. $J$ Neurochem 67:1953-1959.

Hayama T, Ito S, and Ogawa H (1985) Responses of solitary tract nucleus neurons to taste and mechanical stimulations of the oral cavity in decerebrate rats. Exp Brain Res 60:235-242.

Hedman L, Backman H, Stridsman C, Bosson JA, Lundbäck M, Lindberg A, Rönmark E, and Ekerljung L (2018) Association of electronic cigarette use with smoking habits, demographic factors, and respiratory symptoms. JAMA Netw Open 1:e180789.

Henderson BJ, Wall TR, Henley BM, Kim CH, McKinney S, and Lester HA (2017) Menthol Enhances Nicotine Reward-Related Behavior by Potentiating NicotineInduced Changes in nAChR Function, nAChR Upregulation, and DA Neuron Excitability. Neuropsychopharmacology 42:2285-2291.

Henningfield JE and Goldberg SR (1983) Nicotine as a reinforcer in human subjects and laboratory animals. Pharmacol Biochem Behav 19:989-992.

Hentall ID and Gollapudi L (1995) The interpeduncular nucleus regulates nicotine's effects on free-field activity. Neuroreport 6:2469-2472.

Herkenham M and Nauta WJ (1979) Efferent connections of the habenular nucle in the rat. J Comp Neurol 187:19-47.

Hicks JH, Dani JA, and Lester RA (2000) Regulation of the sensitivity of acetylcholine receptors to nicotine in rat habenula neurons. $J$ Physiol 529:579-597.

Higa KK, Grim A, Kamenski ME, et al. (2017) Nicotine withdrawal-induced inattention is absent in alpha7 nAChR knockout mice. Psychopharmacology (Berl) 234:1573-1586.

Hildebrand BE, Nomikos GG, Bondjers C, Nisell M, and Svensson TH (1997) Behavioral manifestations of the nicotine abstinence syndrome in the rat: peripheral versus central mechanisms. Psychopharmacology (Berl) 129:348-356.

Hildebrand BE, Nomikos GG, Hertel P, Schilström B, and Svensson TH (1998) Reduced dopamine output in the nucleus accumbens but not in the medial prefrontal cortex in rats displaying a mecamylamine-precipitated nicotine withdrawal syndrome. Brain Res 779:214-225.

Hildebrand BE, Panagis G, Svensson TH, and Nomikos GG (1999) Behavioral and biochemical manifestations of mecamylamine-precipitated nicotine withdrawal in the rat: role of nicotinic receptors in the ventral tegmental area. Neuropsychopharmacology 21:560-574. 
Hines T, Toney GM, and Mifflin SW (1994) Responses of neurons in the nucleus tractus solitarius to stimulation of heart and lung receptors in the rat. Circ Res 74:1188-1196.

Hnasko TS, Hjelmstad GO, Fields HL, and Edwards RH (2012) Ventral tegmental area glutamate neurons: electrophysiological properties and projections. $J$ Neurosci 32:15076-15085.

Hong S, Jhou TC, Smith M, Saleem KS, and Hikosaka O (2011) Negative reward signals from the lateral habenula to dopamine neurons are mediated by rostromedial tegmental nucleus in primates. J Neurosci 31:11457-11471.

Houser CR, Crawford GD, Barber RP, Salvaterra PM, and Vaughn JE (1983) Organization and morphological characteristics of cholinergic neurons: an immunocytochemical study with a monoclonal antibody to choline acetyltransferase. Brain Res 266:97-119.

Hsu YW, Morton G, Guy EG, Wang SD, and Turner EE (2016) Dorsal Medial Habenula Regulation of Mood-Related Behaviors and Primary Reinforcement by Tachykinin-Expressing Habenula Neurons. eNeuro 3:3.

Hsu YW, Tempest L, Quina LA, Wei AD, Zeng H, and Turner EE (2013) Medial habenula output circuit mediated by $\alpha 5$ nicotinic receptor-expressing GABAergic neurons in the interpeduncular nucleus. $J$ Neurosci 33:18022-18035.

Hu F, Ren J, Zhang JE, Zhong W, and Luo M (2012) Natriuretic peptides block synaptic transmission by activating phosphodiesterase $2 \mathrm{~A}$ and reducing presynaptic PKA activity. Proc Natl Acad Sci USA 109:17681-17686.

Hucho F and Changeux JP (1973) Molecular weight and quaternary structure of the cholinergic receptor protein extracted by detergents from Electrophorus electricus electric tissue. FEBS Lett 38:11-15.

Huganir RL, Delcour AH, Greengard P, and Hess GP (1986) Phosphorylation of the nicotinic acetylcholine receptor regulates its rate of desensitization. Nature 321:774-776.

Hughes JR, Gust SW, Skoog K, Keenan RM, and Fenwick JW (1991) Symptoms of tobacco withdrawal. A replication and extension. Arch Gen Psychiatry 48:52-59.

Hung RJ, McKay JD, Gaborieau V, Boffetta P, Hashibe M, Zaridze D, Mukeria A Szeszenia-Dabrowska N, Lissowska J, Rudnai P, et al. (2008) A susceptibility locus for lung cancer maps to nicotinic acetylcholine receptor subunit genes on 15q25. Nature 452:633-637.

Hussain RJ, Taraschenko OD, and Glick SD (2008) Effects of nicotine, methamphetamine and cocaine on extracellular levels of acetylcholine in the interpeduncular nucleus of rats. Neurosci Lett 440:270-274.

Husson M, Harrington L, Tochon L, Cho Y, Ibañez-Tallon I, Maskos U, and David V (2020) B4-Nicotinic Receptors Are Critically Involved in Reward-Related Behaviors and Self-Regulation of Nicotine Reinforcement. J Neurosci 40:3465-3477.

Huston-Lyons D, Sarkar M, and Kornetsky C (1993) Nicotine and brainstimulation reward: interactions with morphine, amphetamine and pimozide. Pharmacol Biochem Behav 46:453-457.

Ikemoto S, Qin M, and Liu ZH (2006) Primary reinforcing effects of nicotine are triggered from multiple regions both inside and outside the ventral tegmental area. $J$ Neurosci 26:723-730.

Ikemoto S and Wise RA (2002) Rewarding effects of the cholinergic agents carbachol and neostigmine in the posterior ventral tegmental area. J Neurosci 22:9895-9904

Ikemoto S, Witkin BM, and Morales M (2003) Rewarding injections of the cholinergic agonist carbachol into the ventral tegmental area induce locomotion and c-Fos expression in the retrosplenial area and supramammillary nucleus. Brain Res 969:78-87.

Imperato A, Mulas A, and Di Chiara G (1986) Nicotine preferentially stimulates dopamine release in the limbic system of freely moving rats. Eur J Pharmacol 132:337-338.

Ishibashi M, Leonard CS, and Kohlmeier KA (2009) Nicotinic activation of laterodorsal tegmental neurons: implications for addiction to nicotine. Neuropsychopharmacology 34:2529-2547.

Ivanová S and Greenshaw AJ (1997) Nicotine-induced decreases in VTA electrical self-stimulation thresholds: blockade by haloperidol and mecamylamine but not scopolamine or ondansetron. Psychopharmacology (Berl) 134:187-192.

Iwasaki K and Sato M (1981) Neural responses and aversion to bitter stimuli in rats. Chem Senses 6:119-128.

Iyaniwura TT, Wright AE, and Balfour DJ (2001) Evidence that mesoaccumbens dopamine and locomotor responses to nicotine in the rat are influenced by pretreatment dose and strain. Psychopharmacology (Berl) 158:73-79.

Jackson A, Papke RL, and Damaj MI (2018) Pharmacological modulation of the $\alpha 7$ nicotinic acetylcholine receptor in a mouse model of mecamylamine-precipitated nicotine withdrawal. Psychopharmacology (Berl) 235:1897-1905.

Jackson AB, Toma W, Contreras KM, Alkhlaif Y, and Damaj MI (2019) The $\beta 3$ subunit of the nicotinic acetylcholine receptor is required for nicotine withdrawal-induced affective but not physical signs or nicotine reward in mice. Pharmacol Biochem Behav 183:1-5.

Jackson KJ, Marks MJ, Vann RE, Chen X, Gamage TF, Warner JA, and Damaj MI (2010) Role of alpha5 nicotinic acetylcholine receptors in pharmacological and behavioral effects of nicotine in mice. J Pharmacol Exp Ther 334:137-146.

Jackson KJ, Martin BR, Changeux JP, and Damaj MI (2008) Differential role of nicotinic acetylcholine receptor subunits in physical and affective nicotine withdrawal signs. J Pharmacol Exp Ther 325:302-312.

Jackson KJ, McIntosh JM, Brunzell DH, Sanjakdar SS, and Damaj MI (2009) The role of alpha6-containing nicotinic acetylcholine receptors in nicotine reward and withdrawal. J Pharmacol Exp Ther 331:547-554.

Jain A, Kuryatov A, Wang J, Kamenecka TM, and Lindstrom J (2016) Unorthodox Acetylcholine Binding Sites Formed by $\alpha 5$ and $\beta 3$ Accessory Subunits in $\alpha 4 \beta 2^{*}$ Nicotinic Acetylcholine Receptors. J Biol Chem 291:23452-23463.

Jennings C, Gosnell S, Curtis KN, Kosten T, and Salas R (2020) Altered habenula resting state functional connectivity in deprived veteran tobacco smokers: A pilot study. Bull Menninger Clin 84:21-34.
Jensen KP, DeVito EE, Herman AI, Valentine GW, Gelernter J, and Sofuoglu M (2015) A CHRNA5 Smoking Risk Variant Decreases the Aversive Effects of Nicotine in Humans. Neuropsychopharmacology 40:2813-2821.

Jhou TC, Fields HL, Baxter MG, Saper CB, and Holland PC (2009a) The rostromedial tegmental nucleus (RMTg), a GABAergic afferent to midbrain dopamine neurons, encodes aversive stimuli and inhibits motor responses. Neuron 61:786-800.

Jhou TC, Geisler S, Marinelli M, Degarmo BA, and Zahm DS (2009b) The mesopontine rostromedial tegmental nucleus: A structure targeted by the lateral habenula that projects to the ventral tegmental area of Tsai and substantia nigra compacta. J Comp Neurol 513:566-596.

Jin XT, Tucker BR, and Drenan RM (2020) Nicotine Self-Administration Induces Plastic Changes to Nicotinic Receptors in Medial Habenula. eNeuro 7:7.

Johnson JH, Zhao C, James JR, and Rosecrans JA (2000) Individual variability of dopamine release from nucleus accumbens induced by nicotine. Brain Res Bull 51:249-253.

Jonkman S and Markou A (2006) Blockade of nicotinic acetylcholine or dopamine D1-like receptors in the central nucleus of the amygdala or the bed nucleus of the stria terminalis does not precipitate nicotine withdrawal in nicotinedependent rats. Neurosci Lett 400:140-145.

Justice AE, Winkler TW, Feitosa MF, Graff M, Fisher VA, Young K, Barata L, Deng X, Czajkowski J, Hadley D, et al. (2017) Genome-wide meta-analysis of 241,258 adults accounting for smoking behaviour identifies novel loci for obesity traits. Nat Commun 8:14977.

Kaiser SA, Soliakov L, Harvey SC, Luetje CW, and Wonnacott S (1998) Differentia inhibition by alpha-conotoxin-MII of the nicotinic stimulation of $[3 \mathrm{H}]$ dopamine release from rat striatal synaptosomes and slices. J Neurochem 70:1069-1076.

Kemp G and Morley BJ (1986) Ganglionic nAChRs and high-affinity nicotinic binding sites are not equivalent. FEBS Lett 205:265-268.

Kenny PJ, Chartoff E, Roberto M, Carlezon Jr WA, and Markou A (2009) NMDA receptors regulate nicotine-enhanced brain reward function and intravenous nicotine self-administration: role of the ventral tegmental area and centra nucleus of the amygdala. Neuropsychopharmacology 34:266-281.

Kenny PJ, Cheeta S, and File SE (2000) Anxiogenic effects of nicotine in the dorsal hippocampus are mediated by 5 -HT1A and not by muscarinic M1 receptors. Neuropharmacology 39:300-307.

Kenny PJ, Gasparini F, and Markou A (2003) Group II metabotropic and alphaamino-3-hydroxy-5-methyl-4-isoxazole propionate (AMPA)/kainate glutamate receptors regulate the deficit in brain reward function associated with nicotine withdrawal in rats. J Pharmacol Exp Ther 306:1068-1076.

Kenny PJ and Markou A (2001) Neurobiology of the nicotine withdrawal syndrome. Pharmacol Biochem Behav 70:531-549.

Kenny PJ and Markou A (2006) Nicotine self-administration acutely activates brain reward systems and induces a long-lasting increase in reward sensitivity. Neuropsychopharmacology 31:1203-1211.

Kim U and Chang SY (2005) Dendritic morphology, local circuitry, and intrinsic electrophysiology of neurons in the rat medial and lateral habenular nuclei of the epithalamus. J Comp Neurol 483:236-250.

Kimura H, McGeer PL, Peng JH, and McGeer EG (1981) The central cholinergic system studied by choline acetyltransferase immunohistochemistry in the cat. $J$ Comp Neurol 200:151-201.

Kiyatkin EA (2014) Critical role of peripheral sensory systems in mediating the neural effects of nicotine following its acute and repeated exposure. Rev Neurosci 25:207-221.

Klink R, de Kerchove d'Exaerde A, Zoli M, and Changeux JP (2001) Molecular and physiological diversity of nicotinic acetylcholine receptors in the midbrain dopaminergic nuclei. J Neurosci 21:1452-1463.

Knight, C., Howard, P., Baker, C. L. and Marton, J. P. (2009) The CostEffectiveness of an Extended Course $(12+12$ Weeks $)$ of Varenicline Compared with Other Available Smoking Cessation Strategies in the United States: An Extension and Update to the BENESCO Model. Value Health.

Kobayashi Y, Sano Y, Vannoni E, Goto H, Suzuki H, Oba A, Kawasaki H, Kanba S Lipp HP, Murphy NP et al. (2013) Genetic dissection of medial habenulainterpeduncular nucleus pathway function in mice. Front Behav Neurosci 7:17.

Koob GF and Bloom FE (1988) Cellular and molecular mechanisms of drug dependence. Science 242:715-723.

Koob GF and Le Moal M (1997) Drug abuse: hedonic homeostatic dysregulation. Science 278:52-58.

Koppensteiner P, Melani R, and Ninan I (2017) A Cooperative Mechanism Involving $\mathrm{Ca}^{2+}$-Permeable AMPA Receptors and Retrograde Activation of $\mathrm{GABA}_{\mathrm{B}}$ Receptors in Interpeduncular Nucleus Plasticity. Cell Rep 20:1111-1122.

Kulak JM, Nguyen TA, Olivera BM, and McIntosh JM (1997) Alpha-conotoxin MII blocks nicotine-stimulated dopamine release in rat striatal synaptosomes. $J$ Neurosci 17:5263-5270.

Kuryatov A, Berrettini W, and Lindstrom J (2011) Acetylcholine receptor (AChR) $\alpha 5$ subunit variant associated with risk for nicotine dependence and lung cance reduces $(\alpha 4 \beta 2)_{2} \alpha 5$ AChR function. Mol Pharmacol 79:119-125.

Kuryatov A, Onksen J, and Lindstrom J (2008) Roles of accessory subunits in alpha4beta2(*) nicotinic receptors. Mol Pharmacol 74:132-143.

Lai A, Parameswaran N, Khwaja M, Whiteaker P, Lindstrom JM, Fan H, McIntosh JM, Grady SR, and Quik M (2005) Long-term nicotine treatment decreases striatal alpha $6^{*}$ nicotinic acetylcholine receptor sites and function in mice. Mol Pharmacol 67:1639-1647.

Lammel S, Hetzel A, Häckel O, Jones I, Liss B, and Roeper J (2008) Unique properties of mesoprefrontal neurons within a dual mesocorticolimbic dopamine system. Neuron 57:760-773.

Lammel S, Ion DI, Roeper J, and Malenka RC (2011) Projection-specific modulation of dopamine neuron synapses by aversive and rewarding stimuli. Neuron 70:855-862.

Lammel, S., Lim, B. K. and Malenka, R. C. (2014) Reward and aversion in a heterogeneous midbrain dopamine system. Neuropharmacology 76 Pt B, 351-359. 
Lammel S, Lim BK, Ran C, Huang KW, Betley MJ, Tye KM, Deisseroth K, and Malenka RC (2012) Input-specific control of reward and aversion in the ventral tegmental area. Nature 491:212-217.

Lammel S, Steinberg EE, Földy C, Wall NR, Beier K, Luo L, and Malenka RC (2015) Diversity of transgenic mouse models for selective targeting of midbrain dopamine neurons. Neuron 85:429-438.

Laviolette SR, Alexson TO, and van der Kooy D (2002) Lesions of the tegmental pedunculopontine nucleus block the rewarding effects and reveal the aversive effects of nicotine in the ventral tegmental area. $J$ Neurosci 22:8653-8660.

Laviolette SR, Lauzon NM, Bishop SF, Sun N, and Tan H (2008) Dopamine signaling through D1-like versus D2-like receptors in the nucleus accumbens core versus shell differentially modulates nicotine reward sensitivity. $J$ Neurosci 28:8025-8033.

Laviolette, S. R. and van der Kooy, D. (2003a) Blockade of mesolimbic dopamine transmission dramatically increases sensitivity to the rewarding effects of nicotine in the ventral tegmental area. Mol Psychiatry 8, 50-59, 59.

Laviolette SR and van der Kooy D (2003b) The motivational valence of nicotine in the rat ventral tegmental area is switched from rewarding to aversive following blockade of the alpha7-subunit-containing nicotinic acetylcholine receptor. Psychopharmacology (Berl) 166:306-313.

Le Foll B, Wertheim C, and Goldberg SR (2007) High reinforcing efficacy of nicotine in non-human primates. PLoS One 2:e230.

Le Novère N, Corringer PJ, and Changeux JP (2002) The diversity of subunit composition in nAChRs: evolutionary origins, physiologic and pharmacologic consequences. J Neurobiol 53:447-456.

Le Novère N, Zoli M, and Changeux JP (1996) Neuronal nicotinic receptor alpha 6 subunit mRNA is selectively concentrated in catecholaminergic nuclei of the rat brain. Eur J Neurosci 8:2428-2439.

Lecca D, Cacciapaglia F, Valentini V, Gronli J, Spiga S, and Di Chiara G (2006) Preferential increase of extracellular dopamine in the rat nucleus accumbens shell as compared to that in the core during acquisition and maintenance of intravenous nicotine self-administration. Psychopharmacology (Berl) 184:435-446.

Lecca S, Melis M, Luchicchi A, Ennas MG, Castelli MP, Muntoni AL, and Pistis M (2011) Effects of drugs of abuse on putative rostromedial tegmental neurons, inhibitory afferents to midbrain dopamine cells. Neuropsychopharmacology 36:589-602

Lemon CH and Smith DV (2005) Neural representation of bitter taste in the nucleus of the solitary tract. $J$ Neurophysiol 94:3719-3729.

Léna C and Changeux JP (1998) Allosteric nicotinic receptors, human pathologies. $J$ Physiol Paris 92:63-74.

Léna C, Changeux JP, and Mulle C (1993) Evidence for "preterminal" nicotinic receptors on GABAergic axons in the rat interpeduncular nucleus. $J$ Neurosci 13:2680-2688

Lenn NJ (1976) Synapses in the interpeduncular nucleus: electron microscopy of normal and habenula lesioned rats. J Comp Neurol 166:77-99.

Lenn NJ, Wong V, and Hamill GS (1983) Left-right pairing at the crest synapses of rat interpeduncular nucleus. Neuroscience 9:383-389.

Lenoir M and Kiyatkin EA (2011) Critical role of peripheral actions of intravenous nicotine in mediating its central effects. Neuropsychopharmacology 36:2125-2138.

Lerman C, LeSage MG, Perkins KA, O'Malley SS, Siegel SJ, Benowitz NL, and Corrigall WA (2007) Translational research in medication development for nicotine dependence. Nat Rev Drug Discov 6:746-762.

Lester RA and Dani JA (1995) Acetylcholine receptor desensitization induced by nicotine in rat medial habenula neurons. J Neurophysiol 74:195-206.

Leventhal AM, Strong DR, Kirkpatrick MG, Unger JB, Sussman S, Riggs NR, Stone MD, Khoddam R, Samet JM, and Audrain-McGovern J (2015) Association of Electronic Cigarette Use With Initiation of Combustible Tobacco Product Smoking in Early Adolescence. JAMA 314:700-707.

Levin ED, Petro A, Rezvani AH, Pollard N, Christopher NC, Strauss M, Avery J, Nicholson J, and Rose JE (2009) Nicotinic alpha7- or beta2-containing receptor knockout: effects on radial-arm maze learning and long-term nicotine consumption in mice. Behav Brain Res 196:207-213.

Lima LB, Bueno D, Leite F, Souza S, Gonçalves L, Furigo IC, Donato Jr J, and Metzger M (2017) Afferent and efferent connections of the interpeduncular nucleus with special reference to circuits involving the habenula and raphe nuclei. J Comp Neurol 525:2411-2442.

Lips EH, Gaborieau V, McKay JD, et al. (2009) Association between a 15q25 gene variant, smoking quantity and tobacco-related cancers among 17000 individuals. Int $J$ Epidemiol 39:563-77.

Lisoprawski A, Blanc G, and Glowinski J (1981) Activation by stress of the habenulo-interpeduncular substance $\mathrm{P}$ neurons in the rat. Neurosci Lett 25:47-51.

Liu C, Bonaventure P, Lee G, Nepomuceno D, Kuei C, Wu J, Li Q, Joseph V, Sutton SW, Eckert W, et al. (2015) GPR139, an orphan receptor highly enriched in the habenula and septum, is activated by the essential amino acids L-tryptophan and L-phenylalanine. Mol Pharmacol 88:911-925.

Liu L, Zhao-Shea R, McIntosh JM, Gardner PD, and Tapper AR (2012) Nicotine persistently activates ventral tegmental area dopaminergic neurons via nicotinic acetylcholine receptors containing $\alpha 4$ and $\alpha 6$ subunits. Mol Pharmacol 81:541-548.

Liu Q, Han H, Wang M, Yao Y, Wen L, Jiang K, Ma Y, Fan R, Chen J, Su K, et al (2018) Association and cis-mQTL analysis of variants in CHRNA3-A5, CHRNA7, CHRNB2, and CHRNB4 in relation to nicotine dependence in a Chinese Han population. Transl Psychiatry 8:83.

Liu X, Koren AO, Yee SK, Pechnick RN, Poland RE, and London ED (2003) Selfadministration of 5-iodo-A-85380, a beta2-selective nicotinic receptor ligand, by operantly trained rats. Neuroreport 14:1503-1505.

Liu Z and Habener JF (2008) Glucagon-like peptide-1 activation of TCF7L2 dependent Wnt signaling enhances pancreatic beta cell proliferation. $J$ Biol Chem 283:8723-8735.
Lodge DJ and Grace AA (2006) The laterodorsal tegmentum is essential for burst firing of ventral tegmental area dopamine neurons. Proc Natl Acad Sci USA 103:5167-5172.

Lotfipour S, Byun JS, Leach P, Fowler CD, Murphy NP, Kenny PJ, Gould TJ, and Boulter J (2013) Targeted deletion of the mouse $\alpha 2$ nicotinic acetylcholine receptor subunit gene (Chrna2) potentiates nicotine-modulated behaviors. $J$ Neurosci 33:7728-7741.

Lynch WJ and Carroll ME (1999) Regulation of intravenously self-administered nicotine in rats. Exp Clin Psychopharmacol 7:198-207.

Lynch WJ and Carroll ME (2001) Regulation of drug intake. Exp Clin Psychopharmacol 9:131-143.

Madsen HB, Koghar HS, Pooters T, Massalas JS, Drago J, and Lawrence AJ (2015) Role of $\alpha 4-$ and $\alpha 6$-containing nicotinic receptors in the acquisition and maintenance of nicotine self-administration. Addict Biol 20:500-512.

Malin DH, Lake JR, Newlin-Maultsby P, Roberts LK, Lanier JG, Carter VA, Cunningham JS, and Wilson OB (1992) Rodent model of nicotine abstinence syndrome. Pharmacol Biochem Behav 43:779-784.

Mameli-Engvall M, Evrard A, Pons S, Maskos U, Svensson TH, Changeux JP, and Faure P (2006) Hierarchical control of dopamine neuron-firing patterns by nicotinic receptors. Neuron 50:911-921.

Mansvelder HD, Keath JR, and McGehee DS (2002) Synaptic mechanisms underlie nicotine-induced excitability of brain reward areas. Neuron 33:905-919.

Mansvelder HD and McGehee DS (2000) Long-term potentiation of excitatory inputs to brain reward areas by nicotine. Neuron 27:349-357.

Mao D, Perry DC, Yasuda RP, Wolfe BB, and Kellar KJ (2008) The alpha4beta2alpha5 nicotinic cholinergic receptor in rat brain is resistant to upregulation by nicotine in vivo. $J$ Neurochem 104:446-456.

Markou A, Kosten TR, and Koob GF (1998) Neurobiological similarities in depression and drug dependence: a self-medication hypothesis. Neuropsychopharmacology 18:135-174.

Markou A and Paterson NE (2001) The nicotinic antagonist methyllycaconitine has differential effects on nicotine self-administration and nicotine withdrawal in the rat. Nicotine Tob Res 3:361-373.

Marks MJ, Burch JB, and Collins AC (1983) Effects of chronic nicotine infusion on tolerance development and nicotinic receptors. J Pharmacol Exp Ther 226:817-825.

Marks MJ, Grady SR, Salminen O, Paley MA, Wageman CR, McIntosh JM, and Whiteaker P (2014) $\alpha 6 \beta 2 *$-subtype nicotinic acetylcholine receptors are more sensitive than $\alpha 4 \beta 2 *$-subtype receptors to regulation by chronic nicotine administration. $J$ Neurochem 130:185-198

Marks MJ, Meinerz NM, Drago J, and Collins AC (2007) Gene targeting demonstrates that alpha4 nicotinic acetylcholine receptor subunits contribute to expression of diverse $[3 \mathrm{H}]$ epibatidine binding sites and components of biphasic $86 \mathrm{Rb}+$ efflux with high and low sensitivity to stimulation by acetylcholine. Neuropharmacology 53:390-405.

Marks MJ, Pauly JR, Gross SD, Deneris ES, Hermans-Borgmeyer I, Heinemann SF, and Collins AC (1992) Nicotine binding and nicotinic receptor subunit RNA after chronic nicotine treatment. J Neurosci 12:2765-2784.

Marques-Vidal P, Kutalik Z, Paccaud F, Bergmann S, Waeber G, Vollenweider P, and Cornuz J (2011) Variant within the promoter region of the CHRNA3 gene associated with FTN dependence is not related to self-reported willingness to quit smoking. Nicotine Tob Res 13:833-839.

Marshall DL, Redfern PH, and Wonnacott S (1997) Presynaptic nicotinic modulation of dopamine release in the three ascending pathways studied by in vivo microdialysis: comparison of naive and chronic nicotine-treated rats. $J$ Neurochem 68:1511-1519.

Marubio LM, Gardier AM, Durier S, David D, Klink R, Arroyo-Jimenez MM, McIntosh JM, Rossi F, Champtiaux N, Zoli M, et al. (2003) Effects of nicotine in the dopaminergic system of mice lacking the alpha4 subunit of neuronal nicotinic acetylcholine receptors. Eur J Neurosci 17:1329-1337.

Maskos U (2008) The cholinergic mesopontine tegmentum is a relatively neglected nicotinic master modulator of the dopaminergic system: relevance to drugs of abuse and pathology. Br J Pharmacol 153 (Suppl 1):S438-S445.

Maskos U, Molles BE, Pons S, Besson M, Guiard BP, Guilloux JP, Evrard A, Cazala P, Cormier A, Mameli-Engvall M, et al. (2005) Nicotine reinforcement and cognition restored by targeted expression of nicotinic receptors. Nature 436:103-107.

Mathers CD and Loncar D (2006) Projections of global mortality and burden of disease from 2002 to 2030 . PLoS Med 3:e442.

Maurer JJ, Sandager-Nielsen K, and Schmidt HD (2017) Attenuation of nicotine taking and seeking in rats by the stoichiometry-selective alpha4beta2 nicotinic acetylcholine receptor positive allosteric modulator NS9283. Psychopharmacology (Berl) 234:475-484.

Mazzaferro S, Benallegue N, Carbone A, Gasparri F, Vijayan R, Biggin PC, Moron M, and Bermudez I (2011) Additional acetylcholine (ACh) binding site at alpha4 alpha4 interface of (alpha4beta2)2alpha4 nicotinic receptor influences agonist sensitivity. J Biol Chem 286:31043-31054.

Mazzaferro S, Bermudez I, and Sine SM (2019) Potentiation of a neuronal nicotinic receptor via pseudo-agonist site. Cell Mol Life Sci 76:1151-1167.

McCallum SE, Cowe MA, Lewis SW, and Glick SD (2012) $\alpha 3 \beta 4$ nicotinic acetylcholine receptors in the medial habenula modulate the mesolimbic dopaminergic response to acute nicotine in vivo. Neuropharmacology 63:434-440. McClernon FJ, Froeliger B, Rose JE, Kozink RV, Addicott MA, Sweitzer MM Westman EC, and Van Wert DM (2016) The effects of nicotine and non-nicotine smoking factors on working memory and associated brain function. Addict Biol 21:954-961.

McGehee DS, Heath MJ, Gelber S, Devay P, and Role LW (1995) Nicotine enhancement of fast excitatory synaptic transmission in CNS by presynaptic receptors. Science 269:1692-1696.

McGovern DJ, Polter AM, and Root DH(2020) Neurochemical signaling of reward and aversion by ventral tegmental area glutamate neurons. In: bioRxiv. 
McGranahan TM, Patzlaff NE, Grady SR, Heinemann SF, and Booker TK (2011) $\alpha 4 \beta 2$ nicotinic acetylcholine receptors on dopaminergic neurons mediate nicotine reward and anxiety relief. $J$ Neurosci 31:10891-10902.

Melani R, Von Itter R, Jing D, Koppensteiner P, and Ninan I (2019) Opposing effects of an atypical glycinergic and substance $\mathrm{P}$ transmission on interpeduncular nucleus plasticity. Neuropsychopharmacology 44:1828-1836.

Metzger M, Souza R, Lima LB, Bueno D, Goncalves L, Sego C, Donato Jr J, and Shammah-Lagnado SJ (2019) Habenular connections with the dopaminergic and serotonergic system and their role in stress-related psychiatric disorders. Eur $J$ Neurosci 53:65-88.

Mihalak KB, Carroll FI, and Luetje CW (2006) Varenicline is a partial agonist at alpha4beta2 and a full agonist at alpha7 neuronal nicotinic receptors. Mol Pharmacol 70:801-805

Mineur YS, Brunzell DH, Grady SR, Lindstrom JM, McIntosh JM, Marks MJ, King SL, and Picciotto MR (2009) Localized low-level re-expression of highaffinity mesolimbic nicotinic acetylcholine receptors restores nicotine-induced locomotion but not place conditioning. Genes Brain Behav 8:257-266.

Ming D, Ruiz-Avila L, and Margolskee RF (1998) Characterization and solubilization of bitter-responsive receptors that couple to gustducin. Proc Natl Acad Sci USA 95:8933-8938.

Moen JK, DeBaker MC, Myjak JE, Wickman K, and Lee AM (2021) Bidirectional sex-dependent regulation of $\alpha 6$ and $\beta 3$ nicotinic acetylcholine receptors by protein kinase $\mathrm{C} \varepsilon$. Addict Biol 26:e12954.

Mokdad AH, Marks JS, Stroup DF, and Gerberding JL (2004) Actual causes of death in the United States, 2000. JAMA 291:1238-1245.

Molander L, Lunell E, and Fagerström KO (2000) Reduction of tobacco withdrawal symptoms with a sublingual nicotine tablet: a placebo controlled study. Nicotine Tob Res 2:187-191.

Molas S, Zhao-Shea R, Liu L, DeGroot SR, Gardner PD, and Tapper AR (2017) A circuit-based mechanism underlying familiarity signaling and the preference for novelty. Nat Neurosci 20:1260-1268.

Mönnikes H, Lauer G, and Arnold R (1997) Peripheral administration of cholecystokinin activates c-fos expression in the locus coeruleus/subcoeruleus nucleus, dorsal vagal complex and paraventricular nucleus via capsaicinsensitive vagal afferents and CCK-A receptors in the rat. Brain Res 770:277-288.

Monteggia LM, Eisch AJ, Tang MD, Kaczmarek LK, and Nestler EJ (2000) Cloning and localization of the hyperpolarization-activated cyclic nucleotide-gated channel family in rat brain. Brain Res Mol Brain Res 81:129-139.

Morel C, Fattore L, Pons S, Hay YA, Marti F, Lambolez B, De Biasi M, Lathrop M, Fratta W, Maskos U, et al. (2014) Nicotine consumption is regulated by a human polymorphism in dopamine neurons. Mol Psychiatry 19:930-936.

Moroni M, Zwart R, Sher E, Cassels BK, and Bermudez I (2006) alpha4beta2 nicotinic receptors with high and low acetylcholine sensitivity: pharmacology, stoichiometry, and sensitivity to long-term exposure to nicotine. Mol Pharmacol 70:755-768.

Morrison CF and Stephenson JA (1972) The occurrence of tolerance to a central depressant effect of nicotine. Br J Pharmacol 46:151-156.

Morton G, Nasirova N, Sparks DW, Brodsky M, Sivakumaran S, Lambe EK, and Turner EE (2018) Chrna5-expressing neurons in the interpeduncular nucleus mediate aversion primed by prior stimulation or nicotine exposure. $J$ Neurosci 38:6900-6920.

Mugnaini M, Garzotti M, Sartori I, Pilla M, Repeto P, Heidbreder CA, and Tessari M (2006) Selective down-regulation of [(125)I]Y0-alpha-conotoxin MII binding in rat mesostriatal dopamine pathway following continuous infusion of nicotine. Neuroscience 137:565-572.

Mulle C, Choquet D, Korn H, and Changeux JP (1992) Calcium influx through nicotinic receptor in rat central neurons: its relevance to cellular regulation. Neuron 8:135-143.

Mulle C, Vidal C, Benoit P, and Changeux JP (1991) Existence of different subtypes of nicotinic acetylcholine receptors in the rat habenulo-interpeduncular system. $J$ Neurosci 11:2588-2597.

Murray M, Murphy CA, Ross LL, and Haun F (1994) The role of the habenulainterpeduncular pathway in modulating levels of circulating adrenal hormones. Restor Neurol Neurosci 6:301-307.

Naqvi NH and Bechara A (2010) The insula and drug addiction: an interoceptive view of pleasure, urges, and decision-making. Brain Struct Funct 214:435-450.

Naqvi NH, Rudrauf D, Damasio H, and Bechara A (2007) Damage to the insula disrupts addiction to cigarette smoking. Science 315:531-534.

Nashmi R, Xiao C, Deshpande P, McKinney S, Grady SR, Whiteaker P, Huang Q McClure-Begley T, Lindstrom JM, Labarca C, et al. (2007) Chronic nicotine cell specifically upregulates functional alpha $4 *$ nicotinic receptors: basis for both tolerance in midbrain and enhanced long-term potentiation in perforant path. $J$ Neurosci 27:8202-8218.

Natividad LA, Tejeda HA, Torres OV, and O'Dell LE (2010) Nicotine withdrawal produces a decrease in extracellular levels of dopamine in the nucleus accumben that is lower in adolescent versus adult male rats. Synapse 64:136-145.

Naudé J, Tolu S, Dongelmans M, Torquet N, Valverde S, Rodriguez G, Pons S, Maskos U, Mourot A, Marti F, et al. (2016) Nicotinic receptors in the ventral tegmental area promote uncertainty-seeking. Nat Neurosci 19:471-478.

Nelson ME, Kuryatov A, Choi CH, Zhou Y, and Lindstrom J (2003) Alternate stoichiometries of alpha4beta2 nicotinic acetylcholine receptors. Mol Pharmacol 63:332-341.

Nemeth-Coslett R, Henningfield JE, O’Keeffe MK, and Griffiths RR (1986) Effects of mecamylamine on human cigarette smoking and subjective ratings. Psychopharmacology (Berl) 88:420-425.

Neugebauer NM, Henehan RM, Hales CA, and Picciotto MR (2011) Mice lacking the galanin gene show decreased sensitivity to nicotine conditioned place preference. Pharmacol Biochem Behav 98:87-93.

Neugebauer NM, Zhang Z, Crooks PA, Dwoskin LP, and Bardo MT (2006) Effect of a novel nicotinic receptor antagonist, N,N'-dodecane-1,12-diyl-bis-3- picolinium dibromide, on nicotine self-administration and hyperactivity in rats. Psychopharmacology (Berl) 184:426-434.

Ngolab J, Liu L, Zhao-Shea R, Gao G, Gardner PD, and Tapper AR (2015) Functional upregulation of $\alpha 4^{*}$ nicotinic acetylcholine receptors in VTA GABAergic neurons increases sensitivity to nicotine reward. $J$ Neurosci 35:8570-8578.

Niedermaier ON, Smith ML, Beightol LA, Zukowska-Grojec Z, Goldstein DS, and Eckberg DL (1993) Influence of cigarette smoking on human autonomic function. Circulation 88:562-571.

Nisell M, Marcus M, Nomikos GG, and Svensson TH (1997) Differential effects of acute and chronic nicotine on dopamine output in the core and shell of the rat nucleus accumbens. $J$ Neural Transm (Vienna) 104:1-10.

Nisell M, Nomikos GG, and Svensson TH (1994a) Infusion of nicotine in the ventral tegmental area or the nucleus accumbens of the rat differentially affects accumbal dopamine release. Pharmacol Toxicol 75:348-352.

Nisell M, Nomikos GG, and Svensson TH (1994b) Systemic nicotine-induced dopamine release in the rat nucleus accumbens is regulated by nicotinic receptors in the ventral tegmental area. Synapse 16:36-44.

Nomikos GG, Hildebrand BE, Panagis G, and Svensson TH (1999) Nicotine withdrawal in the rat: role of alpha7 nicotinic receptors in the ventral tegmental area. Neuroreport 10:697-702.

Nomikos GG, Schilström B, Hildebrand BE, Panagis G, Grenhoff J, and Svensson TH (2000) Role of alpha7 nicotinic receptors in nicotine dependence and implications for psychiatric illness. Behav Brain Res 113:97-103.

Ntamati NR and Lüscher C (2016) VTA projection neurons releasing GABA and glutamate in the dentate gyrus. eNeuro 3:3

O'Connor EC, Parker D, Rollema H, and Mead AN (2010) The alpha4beta2 nicotinic acetylcholine-receptor partial agonist varenicline inhibits both nicotine self-administration following repeated dosing and reinstatement of nicotine seeking in rats. Psychopharmacology (Berl) 208:365-376.

O'Dell LE, Bruijnzeel AW, Ghozland S, Markou A, and Koob GF (2004) Nicotine withdrawal in adolescent and adult rats. Ann N Y Acad Sci 1021:167-174.

O'Dell LE, Torres OV, Natividad LA, and Tejeda HA (2007) Adolescent nicotine exposure produces less affective measures of withdrawal relative to adult nicotine exposure in male rats. Neurotoxicol Teratol 29:17-22.

O'Leary KT, Loughlin SE, Chen Y, and Leslie FM (2008) Nicotinic acetylcholine receptor subunit mRNA expression in adult and developing rat medullary catecholamine neurons. J Comp Neurol 510:655-672.

Oberg M, Jaakkola MS, Woodward A, Peruga A, and Prüss-Ustün A (2011) Worldwide burden of disease from exposure to second-hand smoke: a retrospective analysis of data from 192 countries. Lancet 377:139-146.

Ogawa, H., Imoto, T. and Hayama, T. (1984) Responsiveness of solitarioparabrachial relay neurons to taste and mechanical stimulation applied to the oral cavity in rats. Experimental brain research. Experimentelle Hirnforschung. Experimentation cerebrale 54, 349-358.

Ohi K, Kuwata A, Shimada T, Kataoka Y, Yasuyama T, Uehara T, and Kawasaki Y (2019) Genome-wide variants shared between smoking quantity and schizophrenia on $15 q 25$ are associated with CHRNA5 expression in the brain. Schizophr Bull 45:813-823.

Olucha-Bordonau FE, Teruel V, Barcia-González J, Ruiz-Torner A, ValverdeNavarro AA, and Martínez-Soriano F (2003) Cytoarchitecture and efferent projections of the nucleus incertus of the rat. J Comp Neurol 464:62-97.

Orejarena MJ, Herrera-Solís A, Pons S, Maskos U, Maldonado R, and Robledo P (2012) Selective re-expression of $\beta 2$ nicotinic acetylcholine receptor subunits in the ventral tegmental area of the mouse restores intravenous nicotine selfadministration. Neuropharmacology 63:235-241.

Otsu Y, Darcq E, Pietrajtis K, Mátyás F, Schwartz E, Bessaih T, Abi Gerges S, Rousseau CV, Grand T, Dieudonné S, et al. (2019) Control of aversion by glycinegated GluN1/GluN3A NMDA receptors in the adult medial habenula. Science 366:250-254.

Otsu Y, Lecca S, Pietrajtis K, Rousseau CV, Marcaggi P, Dugué GP, MailhesHamon C, Mameli M, and Diana MA (2018) Functional principles of posterior septal inputs to the medial habenula. Cell Rep 22:693-705.

Ouagazzal AM, Kenny PJ, and File SE (1999a) Modulation of behaviour on trials 1 and 2 in the elevated plus-maze test of anxiety after systemic and hippocampal administration of nicotine. Psychopharmacology (Berl) 144:54-60.

Ouagazzal AM, Kenny PJ, and File SE (1999b) Stimulation of nicotinic receptors in the lateral septal nucleus increases anxiety. Eur J Neurosci 11:3957-3962.

Oyrer J, Bleakley LE, Richards KL, Maljevic S, Phillips AM, Petrou S, Nowell CJ, and Reid CA (2019) Using a multiplex nucleic acid in situ hybridization technique to determine HCN4 mRNA expression in the adult rodent brain. Front Mol Neurosci 12:211.

Palma E, Maggi L, Barabino B, Eusebi F, and Ballivet M (1999) Nicotinic acetylcholine receptors assembled from the alpha7 and beta3 subunits. $J$ Biol Chem 274:18335-18340.

Pang X, Liu L, Ngolab J, Zhao-Shea R, McIntosh JM, Gardner PD, and Tapper AR (2016) Habenula cholinergic neurons regulate anxiety during nicotine withdrawal via nicotinic acetylcholine receptors. Neuropharmacology 107:294-304.

Pang Y, Kiba H, and Jayaraman A (1993) Acute nicotine injections induce c-fos mostly in non-dopaminergic neurons of the midbrain of the rat. Brain Res $\mathrm{Mol}$ Brain Res 20:162-170.

Paradiso K and Brehm P (1998) Long-term desensitization of nicotinic acetylcholine receptors is regulated via protein kinase A-mediated phosphorylation. J Neurosci 18:9227-9237.

Parker SL, Fu Y, McAllen K, Luo J, McIntosh JM, Lindstrom JM, and Sharp BM (2004) Up-regulation of brain nicotinic acetylcholine receptors in the rat during long-term self-administration of nicotine: disproportionate increase of the alpha6 subunit. Mol Pharmacol 65:611-622.

Parrott AC (1993) Cigarette smoking: effects upon self-rated stress and arousal over the day. Addict Behav 18:389-395. 
Parsons LH and Justice Jr JB (1992) Extracellular concentration and in vivo recovery of dopamine in the nucleus accumbens using microdialysis. $J$ Neurochem 58:212-218.

Passlick S, Thapaliya ER, Chen Z, Richers MT, and Ellis-Davies GCR (2018) Optical probing of acetylcholine receptors on neurons in the medial habenula with a novel caged nicotine drug analogue. J Physiol 596:5307-5318.

Peng C, Engle SE, Yan Y, Weera MM, Berry JN, Arvin MC, Zhao G, McIntosh JM, Chester JA, and Drenan RM (2017) Altered nicotine reward-associated behavior following $\alpha 4 \mathrm{nAChR}$ subunit deletion in ventral midbrain. PLoS One 12:e0182142.

Perez XA, Ly J, McIntosh JM, and Quik M (2012) Long-term nicotine exposure depresses dopamine release in nonhuman primate nucleus accumbens. $J$ Pharmacol Exp Ther 342:335-344.

Pérez-Morales R, González-Zamora A, González-Delgado MF, Calleros Rincón EY, Olivas Calderón EH, Martínez-Ramírez OC, and Rubio J (2018) CHRNA3 rs1051730 and CHRNA5 rs16969968 polymorphisms are associated with heavy smoking, lung cancer, and chronic obstructive pulmonary disease in a mexican population. Ann Hum Genet 82:415-424.

Perkins KA, Lerman C, Coddington S, Jetton C, Karelitz JL, Wilson A, Jennings JR, Ferrell R, Bergen AW, and Benowitz NL (2008) Gene and gene by sex associations with initial sensitivity to nicotine in nonsmokers. Behav Pharmacol 19:630-640.

Perry DC, Mao D, Gold AB, McIntosh JM, Pezzullo JC, and Kellar KJ (2007) Chronic nicotine differentially regulates alpha6- and beta3-containing nicotinic cholinergic receptors in rat brain. J Pharmacol Exp Ther 322:306-315.

Peto R, Darby S, Deo H, Silcocks P, Whitley E, and Doll R (2000) Smoking, smoking cessation, and lung cancer in the UK since 1950: combination of national statistics with two case-control studies. BMJ 321:323-329.

Piasecki TM, Fiore MC, and Baker TB (1998) Profiles in discouragement: two studies of variability in the time course of smoking withdrawal symptoms. $J$ Abnorm Psychol 107:238-251.

Piasecki TM, Jorenby DE, Smith SS, Fiore MC, and Baker TB (2003) Smoking withdrawal dynamics: II. Improved tests of withdrawal-relapse relations. $J$ Abnorm Psychol 112:14-27.

Piasecki TM, Niaura R, Shadel WG, Abrams D, Goldstein M, Fiore MC, and Baker TB (2000) Smoking withdrawal dynamics in unaided quitters. J Abnorm Psychol 109:74-86.

Picciotto MR, Zoli M, Rimondini R, Léna C, Marubio LM, Pich EM, Fuxe K, and Changeux JP (1998) Acetylcholine receptors containing the beta2 subunit are involved in the reinforcing properties of nicotine. Nature 391:173-177.

Pidoplichko VI, DeBiasi M, Williams JT, and Dani JA (1997) Nicotine activates and desensitizes midbrain dopamine neurons. Nature 390:401-404.

Pons S, Fattore L, Cossu G, Tolu S, Porcu E, McIntosh JM, Changeux JP, Maskos U, and Fratta W (2008) Crucial role of alpha4 and alpha6 nicotinic acetylcholine receptor subunits from ventral tegmental area in systemic nicotine selfadministration. $J$ Neurosci 28:12318-12327.

Poorthuis RB, Bloem B, Verhoog MB, and Mansvelder HD (2013) Layer-specific interference with cholinergic signaling in the prefrontal cortex by smoking concentrations of nicotine. J Neurosci 33:4843-4853.

Portugal GS, Kenney JW, and Gould TJ (2008) Beta2 subunit containing acetylcholine receptors mediate nicotine withdrawal deficits in the acquisition of contextual fear conditioning. Neurobiol Learn Mem 89:106-113.

Pritchard WS, Robinson JH, Guy TD, Davis RA, and Stiles MF (1996) Assessing the sensory role of nicotine in cigarette smoking. Psychopharmacology (Berl) 127:55-62.

Qi J, Zhang S, Wang HL, Barker DJ, Miranda-Barrientos J, and Morales M (2016) VTA glutamatergic inputs to nucleus accumbens drive aversion by acting on GABAergic interneurons. Nat Neurosci 19:725-733.

Qin C and Luo M (2009) Neurochemical phenotypes of the afferent and efferent projections of the mouse medial habenula. Neuroscience 161:827-837.

Qin C, Sun Y, Chen JD, and Foreman RD (2005) Gastric electrical stimulation modulates neuronal activity in nucleus tractus solitarii in rats. Auton Neurosci 119:1-8.

Quick MW, Ceballos RM, Kasten M, McIntosh JM, and Lester RA (1999) Alpha3beta4 subunit-containing nicotinic receptors dominate function in rat medial habenula neurons. Neuropharmacology 38:769-783.

Quik M, Polonskaya Y, Gillespie A, Jakowec M, Lloyd GK, and Langston JW (2000) Localization of nicotinic receptor subunit mRNAs in monkey brain by in situ hybridization. J Comp Neurol 425:58-69.

Quina LA, Harris J, Zeng H, and Turner EE (2017) Specific connections of the interpeduncular subnuclei reveal distinct components of the habenulopeduncular pathway. J Comp Neurol 525:2632-2656.

Quina LA, Wang S, Ng L, and Turner EE (2009) Brn3a and Nurr1 mediate a gene regulatory pathway for habenula development. $J$ Neurosci 29:14309-14322.

Ramirez-Latorre J, Yu CR, Qu X, Perin F, Karlin A, and Role L (1996) Functional contributions of alpha5 subunit to neuronal acetylcholine receptor channels. Nature 380:347-351.

Ramón y Cajal S (1953) Histologie du système nerveux de l'homme \& des vertébrés, Consejo Superior de Investigaciones Científicas, Instituto Ramón y Cajal, Madrid.

Raybuck JD and Gould TJ (2009) Nicotine withdrawal-induced deficits in trace fear conditioning in C57BL/6 mice-a role for high-affinity beta2 subunit-containing nicotinic acetylcholine receptors. Eur J Neurosci 29:377-387.

Reavill C, Walther B, Stolerman IP, and Testa B (1990) Behavioural and pharmacokinetic studies on nicotine, cytisine and lobeline. Neuropharmacology 29:619-624.

Ren J, Qin C, Hu F, Tan J, Qiu L, Zhao S, Feng G, and Luo M (2011) Habenula "cholinergic" neurons co-release glutamate and acetylcholine and activate postsynaptic neurons via distinct transmission modes. Neuron 69:445-452.

Ren T and Sagar SM (1992) Induction of c-fos immunostaining in the rat brain after the systemic administration of nicotine. Brain Res Bull 29:589-597.
Reperant C, Pons S, Dufour E, Rollema H, Gardier AM, and Maskos U (2010) Effect of the alpha4beta2* nicotinic acetylcholine receptor partial agonist varenicline on dopamine release in beta 2 knock-out mice with selective re-expression of the beta2 subunit in the ventral tegmental area. Neuropharmacology 58:346-350.

Reuben M, Boye S, and Clarke PB (2000) Nicotinic receptors modulating somatodendritic and terminal dopamine release differ pharmacologically. Eur $J$ Pharmacol 393:39-49.

Reus VI, Obach RS, Coe JW, Faessel H, Rollema H, Watsky E, and Reeves K (2007) Varenicline: new treatment with efficacy in smoking cessation. Drugs Today (Barc) 43:65-75.

Rice ME and Cragg SJ (2004) Nicotine amplifies reward-related dopamine signals in striatum. Nat Neurosci 7:583-584

Rinaman L, Baker EA, Hoffman GE, Stricker EM, and Verbalis JG (1998) Medullary c-Fos activation in rats after ingestion of a satiating meal. Am $J$ Physiol 275:R262-R268.

Risner ME and Goldberg SR (1983) A comparison of nicotine and cocaine selfadministration in the dog: fixed-ratio and progressive-ratio schedules of intravenous drug infusion. J Pharmacol Exp Ther 224:319-326.

Rodd ZA, Bell RL, Kuc KA, Zhang Y, Murphy JM, and McBride WJ (2005) Intracranial self-administration of cocaine within the posterior ventral tegmental area of Wistar rats: evidence for involvement of serotonin-3 receptors and dopamine neurons. J Pharmacol Exp Ther 313:134-145.

Rodd-Henricks ZA, McKinzie DL, Crile RS, Murphy JM, and McBride WJ (2000) Regional heterogeneity for the intracranial self-administration of ethanol within the ventral tegmental area of female Wistar rats. Psychopharmacology (Berl) 149:217-224.

Rodrigo J, Springall DR, Uttenthal O, Bentura ML, Abadia-Molina F, RiverosMoreno V, Martínez-Murillo R, Polak JM, and Moncada S (1994) Localization of nitric oxide synthase in the adult rat brain. Philos Trans $R$ Soc Lond B Biol Sci 345:175-221.

Rollema H, Chambers LK, Coe JW, Glowa J, Hurst RS, Lebel LA, Lu Y, Mansbach RS, Mather RJ, Rovetti CC, et al. (2007) Pharmacological profile of the alpha4beta2 nicotinic acetylcholine receptor partial agonist varenicline, an effective smoking cessation aid. Neuropharmacology 52:985-994.

Rollema H, Shrikhande A, Ward KM, Tingley 3rd FD, Coe JW, O’Neill BT, Tseng E, Wang EQ, Mather RJ, Hurst RS, et al. (2010) Pre-clinical properties of the alpha4beta2 nicotinic acetylcholine receptor partial agonists varenicline, cytisine and dianicline translate to clinical efficacy for nicotine dependence. $\mathrm{Br} J$ Pharmacol 160:334-345.

Root DH, Mejias-Aponte CA, Qi J, and Morales M (2014a) Role of glutamatergic projections from ventral tegmental area to lateral habenula in aversive conditioning. J Neurosci 34:13906-13910.

Root DH, Mejias-Aponte CA, Zhang S, Wang HL, Hoffman AF, Lupica CR, and Morales M (2014b) Single rodent mesohabenular axons release glutamate and GABA. Nat Neurosci 17:1543-1551.

Rose JE, Behm FM, and Levin ED (1993) Role of nicotine dose and sensory cues in the regulation of smoke intake. Pharmacol Biochem Behav 44:891-900.

Rose JE, Dehkordi O, Manaye KF, Millis RM, Cianaki SA, and Jayam-Trouth A (2016) The sensory impact of nicotine on noradrenergic and dopaminergic neurons of the nicotine reward - addiction neurocircuitry. J Addict Res Ther 7:7.

Rose JE, Tashkin DP, Ertle A, Zinser MC, and Lafer R (1985) Sensory blockade of smoking satisfaction. Pharmacol Biochem Behav 23:289-293.

Rossi S, Singer S, Shearman E, Sershen H, and Lajtha A (2005) The effects of cholinergic and dopaminergic antagonists on nicotine-induced cerebral neurotransmitter changes. Neurochem Res 30:541-558.

Rostron BL, Chang CM, and Pechacek TF (2014) Estimation of cigarette smokingattributable morbidity in the United States. JAMA Intern Med 174:1922-1928.

Saccone SF, Hinrichs AL, Saccone NL, Chase GA, Konvicka K, Madden PA Breslau N, Johnson EO, Hatsukami D, Pomerleau O, et al. (2007) Cholinergic nicotinic receptor genes implicated in a nicotine dependence association study targeting 348 candidate genes with 3713 SNPs. Hum Mol Genet 16:36-49.

Sachs DP and Leischow SJ (1991) Pharmacologic approaches to smoking cessation. Clin Chest Med 12:769-791.

Sakoori K and Murphy NP (2009) Enhanced nicotine sensitivity in nociceptin/ orphanin FQ receptor knockout mice. Neuropharmacology 56:896-904.

Salas R, Cook KD, Bassetto L, and De Biasi M (2004a) The alpha3 and beta4 nicotinic acetylcholine receptor subunits are necessary for nicotine-induced seizures and hypolocomotion in mice. Neuropharmacology 47:401-407.

Salas R, Main A, Gangitano D, and De Biasi M (2007) Decreased withdrawal symptoms but normal tolerance to nicotine in mice null for the alpha7 nicotinic acetylcholine receptor subunit. Neuropharmacology 53:863-869.

Salas R, Pieri F, and De Biasi M (2004b) Decreased signs of nicotine withdrawal in mice null for the beta4 nicotinic acetylcholine receptor subunit. $J$ Neurosci 24:10035-10039.

Salas R, Sturm R, Boulter J, and De Biasi M (2009) Nicotinic receptors in the habenulo-interpeduncular system are necessary for nicotine withdrawal in mice. J Neurosci 29:3014-3018.

Salminen O, Drapeau JA, McIntosh JM, Collins AC, Marks MJ, and Grady SR (2007) Pharmacology of alpha-conotoxin MII-sensitive subtypes of nicotinic acetylcholine receptors isolated by breeding of null mutant mice. Mol Pharmacol 71:1563-1571.

Salminen O, Murphy KL, McIntosh JM, Drago J, Marks MJ, Collins AC, and Grady SR (2004) Subunit composition and pharmacology of two classes of striatal presynaptic nicotinic acetylcholine receptors mediating dopamine release in mice. Mol Pharmacol 65:1526-1535.

Salminen O, Seppä T, Gäddnäs H, and Ahtee L (2000) Effect of acute nicotine on Fos protein expression in rat brain during chronic nicotine and its withdrawal. Pharmacol Biochem Behav 66:87-93.

Sánchez-Catalán MJ, Hipólito L, Zornoza T, Polache A, and Granero L (2009) Motor stimulant effects of ethanol and acetaldehyde injected into the posterior 
ventral tegmental area of rats: role of opioid receptors. Psychopharmacology (Berl) 204:641-653.

Sanjakdar SS, Maldoon PP, Marks MJ, Brunzell DH, Maskos U, McIntosh JM, Bowers MS, and Damaj MI (2015) Differential roles of $\alpha 6 \beta 2^{*}$ and $\alpha 4 \beta 2^{*}$ neuronal nicotinic receptors in nicotine- and cocaine-conditioned reward in mice. Neuropsychopharmacology 40:350-360.

Santoro B, Chen S, Luthi A, Pavlidis P, Shumyatsky GP, Tibbs GR, and Siegelbaum SA (2000) Molecular and functional heterogeneity of hyperpolarization-activated pacemaker channels in the mouse CNS. J Neurosci 20:5264-5275.

Sargent PB (1993) The diversity of neuronal nicotinic acetylcholine receptors. Annu Rev Neurosci 16:403-443.

Sartor CE, Lessov-Schlaggar CN, Scherrer JF, Bucholz KK, Madden PA, Pergadia ML, Grant JD, Jacob T, and Xian H (2010) Initial response to cigarettes predicts rate of progression to regular smoking: findings from an offspring-of-twins design. Addict Behav 35:771-778.

Sastry BR (1978) Effects of substance P, acetylcholine and stimulation of habenula on rat interpeduncular neuronal activity. Brain Res 144:404-410.

Schaefer GJ and Michael RP (1986) Task-specific effects of nicotine in rats. Intracranial self-stimulation and locomotor activity. Neuropharmacology 25:125-131.

Schiffer H, Atienza J, Reichard H, et al. (2020) The selective gpr139 agonist tak041 reverses anhedonia and social interaction deficits in rodent models related to negative symptoms in schizophrenia. Schizophr Bull 46:S106-S107.

Schilström B, Nomikos GG, Nisell M, Hertel P, and Svensson TH (1998) N-methyl$\mathrm{D}$-aspartate receptor antagonism in the ventral tegmental area diminishes the systemic nicotine-induced dopamine release in the nucleus accumbens Neuroscience 82:781-789.

Schneider NG and Jarvik ME (1984) Time course of smoking withdrawal symptoms as a function of nicotine replacement. Psychopharmacology (Berl) 82:143-144.

Scholze P and Huck S (2020) The alpha5 nicotinic acetylcholine receptor subunit differentially modulates alpha4beta $\left.2{ }^{*}\right)$ and alpha3beta4 $\left(^{*}\right)$ receptors. Front Synaptic Neurosci 12:607959.

Schultz W (1986) Responses of midbrain dopamine neurons to behavioral trigger stimuli in the monkey. $J$ Neurophysiol 56:1439-1461.

Schuster RM, Pachas GN, Stoeckel L, Cather C, Nadal M, Mischoulon D, Schoenfeld DA, Zhang H, Ulysse C, Dodds EB, et al. (2018) Phase IIb trial of an $\alpha 7$ nicotinic receptor partial agonist with and without nicotine patch for withdrawal-associated cognitive deficits and tobacco abstinence. J Clin Psychopharmacol 38:307-316.

Schwartz GJ (2000) The role of gastrointestinal vagal afferents in the control of food intake: current prospects. Nutrition 16:866-873.

Schwartz RD, Lehmann J, and Kellar KJ (1984) Presynaptic nicotinic cholinergic receptors labeled by $[3 \mathrm{H}]$ acetylcholine on catecholamine and serotonin axons in brain. J Neurochem 42:1495-1498.

Sciaccaluga M, Moriconi C, Martinello K, Catalano M, Bermudez I, Stitzel JA Maskos U, and Fucile S (2015) Crucial role of nicotinic $\alpha 5$ subunit variants for $\mathrm{Ca} 2+$ fluxes in ventral midbrain neurons. FASEB J 29:3389-3398.

Scott TR, Yaxley S, Sienkiewicz ZJ, and Rolls ET (1986) Gustatory responses in the nucleus tractus solitarius of the alert cynomolgus monkey. $J$ Neurophysiol $\mathbf{5 5}: 182-200$.

Sellings LH, Baharnouri G, McQuade LE, and Clarke PB (2008) Rewarding and aversive effects of nicotine are segregated within the nucleus accumbens. Eur $J$ Neurosci 28:342-352.

Shao W, Wang D, Chiang YT, Ip W, Zhu L, Xu F, Columbus J, Belsham DD, Irwin DM, Zhang H, et al. (2013) The Wnt signaling pathway effector TCF7L2 controls gut and brain proglucagon gene expression and glucose homeostasis. Diabetes 62:789-800.

Sheffield EB, Quick MW, and Lester RA (2000) Nicotinic acetylcholine receptor subunit mRNA expression and channel function in medial habenula neurons. Neuropharmacology 39:2591-2603.

Sherafat Y, Bautista M, Fowler JP, Chen E, Ahmed A, and Fowler CD (2020) The interpeduncular-ventral hippocampus pathway mediates active stress coping and natural reward. eNeuro 7:7.

Sherva R, Wilhelmsen K, Pomerleau CS, Chasse SA, Rice JP, Snedecor SM, Bierut LJ, Neuman RJ, and Pomerleau OF (2008) Association of a single nucleotide polymorphism in neuronal acetylcholine receptor subunit alpha 5 (CHRNA5) with smoking status and with 'pleasurable buzz' during early experimentation with smoking. Addiction 103:1544-1552.

Shibata $\mathrm{H}$ and Suzuki T (1984) Efferent projections of the interpeduncular complex in the rat, with special reference to its subnuclei: a retrograde horseradish peroxidase study. Brain Res 296:345-349.

Shiffman SM and Jarvik ME (1976) Smoking withdrawal symptoms in two weeks of abstinence. Psychopharmacology (Berl) 50:35-39.

Shih PY, Engle SE, Oh G, Deshpande P, Puskar NL, Lester HA, and Drenan RM (2014) Differential expression and function of nicotinic acetylcholine receptors in subdivisions of medial habenula. J Neurosci 34:9789-9802.

Shih PY, McIntosh JM, and Drenan RM (2015) Nicotine dependence reveals distinct responses from neurons and their resident nicotinic receptors in medial habenula. Mol Pharmacol 88:1035-1044.

Shippenberg TS, Bals-Kubik R, and Herz A (1993) Examination of the neurochemical substrates mediating the motivational effects of opioids: role of the mesolimbic dopamine system and D-1 vs. D-2 dopamine receptors. $J$ Pharmacol Exp Ther 265:53-59.

Shoaib M and Bizarro L (2005) Deficits in a sustained attention task following nicotine withdrawal in rats. Psychopharmacology (Berl) 178:211-222.

Shoaib M, Schindler CW, and Goldberg SR (1997) Nicotine self-administration in rats: strain and nicotine pre-exposure effects on acquisition. Psychopharmacology (Berl) 129:35-43.

Shumake J, Edwards E, and Gonzalez-Lima F (2003) Opposite metabolic changes in the habenula and ventral tegmental area of a genetic model of helpless behavior. Brain Res 963:274-281.
Simons CT, Boucher Y, Carstens MI, and Carstens E (2006) Nicotine suppression of gustatory responses of neurons in the nucleus of the solitary tract. $J$ Neurophysiol 96:1877-1886.

Singer G and Wallace M (1984) Effects of 6-OHDA lesions in the nucleus accumbens on the acquisition of self injection of heroin under schedule and non schedule conditions in rats. Pharmacol Biochem Behav 20:807-809.

Slimak MA, Ables JL, Frahm S, Antolin-Fontes B, Santos-Torres J, Moretti M, Gotti C, and Ibañez-Tallon I (2014) Habenular expression of rare missense variants of the $\beta 4$ nicotinic receptor subunit alters nicotine consumption. Front Hum Neurosci 8:12.

Smaha LA and Kaelber WW (1973) Efferent fiber projections of the habenula and the interpeduncular nucleus. An experimental study in the opossum and cat. Exp Brain Res 16:291-308.

Soria-Gómez E, Busquets-Garcia A, Hu F, Mehidi A, Cannich A, Roux L, Louit I, Alonso L, Wiesner T, Georges F et al (2015) Habenular CB1 receptors control the expression of aversive memories. Neuron 88:306-313.

Sotomayor-Zárate R, Gysling K, Busto UE, Cassels BK, Tampier L, and Quintanilla ME (2013) Varenicline and cytisine: two nicotinic acetylcholine receptor ligands reduce ethanol intake in University of Chile bibulous rats. Psychopharmacology (Berl) 227:287-298.

Spealman RD (1983) Maintenance of behavior by postponement of scheduled injections of nicotine in squirrel monkeys. $J$ Pharmacol Exp Ther 227:154-159.

Spealman RD and Goldberg SR (1978) Drug self-administration by laboratory animals: control by schedules of reinforcement. Annu Rev Pharmacol Toxicol 18:313-339.

Spealman RD and Goldberg SR (1982) Maintenance of schedule-controlled behavior by intravenous injections of nicotine in squirrel monkeys. $J$ Pharmacol Exp Ther 223:402-408.

Sperlágh B, Kittel A, Lajtha A, and Vizi ES (1995) ATP acts as fast neurotransmitter in rat habenula: neurochemical and enzymecytochemical evidence. Neuroscience 66:915-920.

Sperlágh B, Maglóczky Z, Vizi ES, and Freund TF (1998) The triangular septal nucleus as the major source of ATP release in the rat habenula: a combined neurochemical and morphological study. Neuroscience 86:1195-1207.

Spina L, Fenu S, Longoni R, Rivas E, and Di Chiara G (2006) Nicotine-conditioned single-trial place preference: selective role of nucleus accumbens shell dopamine D1 receptors in acquisition. Psychopharmacology (Berl) 184:447-455.

Staley JK, Krishnan-Sarin S, Cosgrove KP, Krantzler E, Frohlich E, Perry E, Dubin JA, Estok K, Brenner E, Baldwin RM, et al. (2006) Human tobacco smokers in early abstinence have higher levels of beta2* nicotinic acetylcholine receptors than nonsmokers. $J$ Neurosci 26:8707-8714.

Stamatakis AM, Jennings JH, Ung RL, Blair GA, Weinberg RJ, Neve RL, Boyce F Mattis J, Ramakrishnan C, Deisseroth K, et al. (2013) A unique population of ventral tegmental area neurons inhibits the lateral habenula to promote reward. Neuron 80:1039-1053.

Statistics NCH (2018) National Health Interview Survey, Centers for Disease Control and Prevention, Atlanta, GA

Steensland P, Simms JA, Holgate J, Richards JK, and Bartlett SE (2007) Varenicline, an alpha4beta2 nicotinic acetylcholine receptor partial agonist, selectively decreases ethanol consumption and seeking. Proc Natl Acad Sci USA 104:12518-12523.

Steidl S, O'Sullivan S, Pilat D, Bubula N, Brown J, and Vezina P (2017a) Operant responding for optogenetic excitation of LDTg inputs to the VTA requires D1 and D2 dopamine receptor activation in the NAcc. Behav Brain Res 333:161-170.

Steidl S and Veverka K (2015) Optogenetic excitation of LDTg axons in the VTA reinforces operant responding in rats. Brain Res 1614:86-93.

Steidl S, Wang H, Ordonez M, Zhang S, and Morales M (2017b) Optogenetic excitation in the ventral tegmental area of glutamatergic or cholinergic inputs from the laterodorsal tegmental area drives reward. Eur J Neurosci 45:559-571.

Stephenson DT, Coskran TM, Kelly MP, Kleiman RJ, Morton D, O’Neill SM Schmidt CJ, Weinberg RJ, and Menniti FS (2012) The distribution of phosphodiesterase 2A in the rat brain. Neuroscience 226:145-155.

Stephenson DT, Coskran TM, Wilhelms MB, Adamowicz WO, O'Donnell MM, Muravnick KB, Menniti FS, Kleiman RJ, and Morton D (2009) Immunohistochemical localization of phosphodiesterase $2 \mathrm{~A}$ in multiple mammalian species. $J$ Histochem Cytochem 57:933-949.

Stevens VL, Bierut LJ, Talbot JT, Wang JC, Sun J, Hinrichs AL, Thun MJ, Goate A, and Calle EE (2008) Nicotinic receptor gene variants influence susceptibility to heavy smoking. Cancer Epidemiol Biomarkers Prev 17:3517-3525.

Stoker AK, Marks MJ, and Markou A (2015) Null mutation of the $\beta 2$ nicotinic acetylcholine receptor subunit attenuates nicotine withdrawal-induced anhedonia in mice. Eur J Pharmacol 753:146-150.

Stoker AK, Olivier B, and Markou A (2012) Role of $\alpha 7$ - and $\beta 4$-containing nicotinic acetylcholine receptors in the affective and somatic aspects of nicotine withdrawal: studies in knockout mice. Behav Genet 42:423-436.

Stolerman IP, Fink R, and Jarvik ME (1973) Acute and chronic tolerance to nicotine measured by activity in rats. Psychopharmacology (Berl) 30:329-342.

Stolerman IP and Jarvis MJ (1995) The scientific case that nicotine is addictive. Psychopharmacology (Berl) 117:2-10, discussion 14-20.

Stoveken HM, Zucca S, Masuho I, Grill B, and Martemyanov KA (2020) The orphan receptor GPR139 signals via $\mathrm{G}_{\mathrm{q} / 11}$ to oppose opioid effects. J Biol Chem 295:10822-10830.

Subramaniyan M and Dani JA (2015) Dopaminergic and cholinergic learning mechanisms in nicotine addiction. Ann N Y Acad Sci 1349:46-63.

Sung YJ, Winkler TW, de Las Fuentes L, Bentley AR, Brown MR, Kraja AT, Schwander K, Ntalla I, Guo X, Franceschini N, et al.; CHARGE Neurology Working Group; COGENT-Kidney Consortium; GIANT Consortium; Lifelines Cohort Study (2018) A large-scale multi-ancestry genome-wide study accounting for smoking behavior identifies multiple significant loci for blood pressure. Am J Hum Genet 102:375-400. 
Szőnyi A, Sos KE, Nyilas R, Schlingloff D, Domonkos A, Takács VT, Pósfai B, Hegedüs P, Priestley JB, Gundlach AL, et al. (2019) Brainstem nucleus incertus controls contextual memory formation. Science 364:364

Tan H, Bishop SF, Lauzon NM, Sun N, and Laviolette SR (2009) Chronic nicotine exposure switches the functional role of mesolimbic dopamine transmission in the processing of nicotine's rewarding and aversive effects. Neuropharmacology 56:741-751.

Tapia L, Kuryatov A, and Lindstrom J (2007) Ca2+ permeability of the (alpha4)3(beta2)2 stoichiometry greatly exceeds that of (alpha4)2(beta2)3 human acetylcholine receptors. Mol Pharmacol 71:769-776.

Tapper AR, McKinney SL, Nashmi R, Schwarz J, Deshpande P, Labarca C, Whiteaker P, Marks MJ, Collins AC, and Lester HA (2004) Nicotine activation of alpha4* receptors: sufficient for reward, tolerance, and sensitization. Science 306:1029-1032.

Thiruchselvam T, Malik S, and Le Foll B (2017) A review of positron emission tomography studies exploring the dopaminergic system in substance use with a focus on tobacco as a co-variate. Am J Drug Alcohol Abuse 43:197-214.

Thompson R (1960) Interpeduncular nucleus and avoidance conditioning in the rat. Science 132:1551-1553.

Thorgeirsson TE, Geller F, Sulem P, Rafnar T, Wiste A, Magnusson KP, Manolescu A, Thorleifsson G, Stefansson H, Ingason A, et al. (2008) A variant associated with nicotine dependence, lung cancer and peripheral arterial disease. Nature 452:638-642.

Thorgeirsson TE, Gudbjartsson DF, Surakka I, Vink JM, Amin N, Geller F, Sulem P, Rafnar T, Esko T, Walter S, et al.; ENGAGE Consortium (2010) Sequence variants at CHRNB3-CHRNA6 and CYP2A6 affect smoking behavior. Nat Genet 42:448-453.

Threlfell S, Lalic T, Platt NJ, Jennings KA, Deisseroth K, and Cragg SJ (2012) Striatal dopamine release is triggered by synchronized activity in cholinergic interneurons. Neuron 75:58-64.

Timmermann DB, Sandager-Nielsen K, Dyhring T, Smith M, Jacobsen AM, Nielsen Eł, Grunnet M, Christensen JK, Peters D, Kohlhaas K, et al. (2012) Augmentation of cognitive function by NS9283, a stoichiometry-dependent positive allosteric modulator of $\alpha 2$ - and $\alpha 4$-containing nicotinic acetylcholine receptors. Br J Pharmacol 167:164-182.

Tolu S, Eddine R, Marti F, David V, Graupner M, Pons S, Baudonnat M, Husson M, Besson M, Reperant C, et al. (2013) Co-activation of VTA DA and GABA neurons mediates nicotine reinforcement. Mol Psychiatry 18:382-393.

Trigo JM, Zimmer A, and Maldonado R (2009) Nicotine anxiogenic and rewarding effects are decreased in mice lacking beta-endorphin. Neuropharmacology 56:1147-1153.

Tuesta LM, Chen Z, Duncan A, Fowler CD, Ishikawa M, Lee BR, Liu XA, Lu Q, Cameron M, Hayes MR, et al. (2017) GLP-1 acts on habenular avoidance circuits to control nicotine intake. Nat Neurosci 20:708-716.

Tuesta LM, Fowler CD, and Kenny PJ (2011) Recent advances in understanding nicotinic receptor signaling mechanisms that regulate drug self-administration behavior. Biochem Pharmacol 82:984-995.

van Bloemendaal L, Ten Kulve JS, la Fleur SE, Ijzerman RG, and Diamant M (2014) Effects of glucagon-like peptide 1 on appetite and body weight: focus on the CNS. J Endocrinol 221:T1-T16.

Vazquez-Roque MI, Camilleri M, Vella A, Carlson P, Laugen J, and Zinsmeister AR (2011) Association of TCF7L2 allelic variations with gastric function, satiation, and GLP-1 levels. Clin Transl Sci 4:183-187.

Viswanath H, Carter AQ, Baldwin PR, Molfese DL, and Salas R (2014) The medial habenula: still neglected. Front Hum Neurosci 7:931.

Wada E, Wada K, Boulter J, Deneris E, Heinemann S, Patrick J, and Swanson LW (1989) Distribution of alpha 2, alpha 3, alpha 4, and beta 2 neuronal nicotinic receptor subunit mRNAs in the central nervous system: a hybridization histochemical study in the rat. J Comp Neurol 284:314-335.

Wada K, Ballivet M, Boulter J, Connolly J, Wada E, Deneris ES, Swanson LW, Heinemann S, and Patrick J (1988) Functional expression of a new pharmacologica subtype of brain nicotinic acetylcholine receptor. Science 240:330-334.

Wageman CR, Marks MJ, and Grady SR (2014) Effectiveness of nicotinic agonists as desensitizers at presynaptic $\alpha 4 \beta 2$ - and $\alpha 4 \alpha 5 \beta 2$-nicotinic acetylcholine receptors. Nicotine Tob Res 16:297-305

Wagner F, Bernard R, Derst C, French L, and Veh RW (2016) Microarray analysis of transcripts with elevated expressions in the rat medial or lateral habenula suggest fast GABAergic excitation in the medial habenula and habenular involvement in the regulation of feeding and energy balance. Brain Struct Funct 221:4663-4689.

Wall TR, Henderson BJ, Voren G, Wageman CR, Deshpande P, Cohen BN, Grady SR, Marks MJ, Yohannes D, Kenny PJ, et al. (2017) TC299423, a novel agonist for nicotinic acetylcholine receptors. Front Pharmacol 8:641.

Walters CL, Brown S, Changeux JP, Martin B, and Damaj MI (2006) The beta2 but not alpha7 subunit of the nicotinic acetylcholine receptor is required for nicotineconditioned place preference in mice. Psychopharmacology (Berl) 184:339-344

Walters CL, Cleck JN, Kuo YC, and Blendy JA (2005) Mu-opioid receptor and CREB activation are required for nicotine reward. Neuron 46:933-943.

Wang D, Stoveken HM, Zucca S, Dao M, Orlandi C, Song C, Masuho I, Johnston C, Opperman KJ, Giles AC, et al. (2019) Genetic behavioral screen identifies an orphan anti-opioid system. Science 365:1267-1273.

Wang F, Gerzanich V, Wells GB, Anand R, Peng X, Keyser K, and Lindstrom J (1996) Assembly of human neuronal nicotinic receptor alpha5 subunits with alpha3, beta2, and beta4 subunits. $J$ Biol Chem 271:17656-17665.

Wang F, Nelson ME, Kuryatov A, Olale F, Cooper J, Keyser K, and Lindstrom J (1998) Chronic nicotine treatment up-regulates human alpha3 beta2 but not alpha3 beta4 acetylcholine receptors stably transfected in human embryonic kidney cells. J Biol Chem 273:28721-28732.
Wang J, Blasio A, Chapman HL, Doebelin C, Liaw V, Kuryatov A, Giovanetti SM, Lindstrom J, Lin L, Cameron MD, et al. (2020) Promoting activity of $(\alpha 4)_{3}(\beta 2)_{2}$ nicotinic cholinergic receptors reduces ethanol consumption. Neuropsychopharmacology 45:301-308.

Wang J, Kuryatov A, Sriram A, Jin Z, Kamenecka TM, Kenny PJ, and Lindstrom J (2015) An accessory agonist binding site promotes activation of $\alpha 4 \beta 2 *$ nicotinic acetylcholine receptors. J Biol Chem 290:13907-13918.

Wang JC, Cruchaga C, Saccone NL, Bertelsen S, Liu P, Budde JP, Duan W, Fox L, Grucza RA, Kern J, et al.; COGEND collaborators and GELCC collaborators (2009) Risk for nicotine dependence and lung cancer is conferred by mRNA expression levels and amino acid change in CHRNA5. Hum Mol Genet 18:3125-3135.

Wang S, D van der Vaart A, Xu Q, Seneviratne C, Pomerleau OF, Pomerleau CS, Payne TJ, Ma JZ, and Li MD (2014) Significant associations of CHRNA2 and CHRNA6 with nicotine dependence in European American and African American populations. Hum Genet 133:575-586.

Ware JJ, van den Bree MB, and Munafò MR (2011) Association of the CHRNA5A3-B4 gene cluster with heaviness of smoking: a meta-analysis. Nicotine Tob Res 13:1167-1175

Watkins SS, Epping-Jordan MP, Koob GF, and Markou A (1999) Blockade of nicotine self-administration with nicotinic antagonists in rats. Pharmacol Biochem Behav 62:743-751.

Watkins SS, Stinus L, Koob GF, and Markou A (2000) Reward and somatic changes during precipitated nicotine withdrawal in rats: centrally and peripherally mediated effects. J Pharmacol Exp Ther 292:1053-1064.

Weiss RB, Baker TB, Cannon DS, von Niederhausern A, Dunn DM, Matsunami N, Singh NA, Baird L, Coon H, McMahon WM, et al. (2008) A candidate gene approach identifies the CHRNA5-A3-B4 region as a risk factor for age-dependent nicotine addiction. PLoS Genet 4:e1000125.

Wen L, Yang Z, Cui W, and Li MD (2016) Crucial roles of the CHRNB3-CHRNA6 gene cluster on chromosome 8 in nicotine dependence: update and subjects for future research. Transl Psychiatry 6:e843.

West RJ, Russell MA, Jarvis MJ, and Feyerabend C (1984) Does switching to an ultralow nicotine cigarette induce nicotine withdrawal effects? Psychopharmacology (Berl) 84:120-123.

Whiteaker P, McIntosh JM, Luo S, Collins AC, and Marks MJ (2000) 125I-alphaconotoxin MII identifies a novel nicotinic acetylcholine receptor population in mouse brain. Mol Pharmacol 57:913-925.

WHO (2008) WHO Report on the Global Tobacco Epidemic, 2008: the MPOWER package, World Health Organization, Geneva.

Williams JM, Gandhi KK, Lu SE, Steinberg ML, and Benowitz NL (2013) Rapid smoking may not be aversive in schizophrenia. Nicotine Tob Res 15:262-266.

Willing AE and Berthoud HR (1997) Gastric distension-induced c-fos expression in catecholaminergic neurons of rat dorsal vagal complex. Am $J$ Physiol 272:R59-R67.

Winzer-Serhan UH and Leslie FM (1997) Codistribution of nicotinic acetylcholine receptor subunit alpha3 and beta4 mRNAs during rat brain development. $J$ Comp Neurol 386:540-554.

Wise RA, Bauco P, Carlezon Jr WA, and Trojniar W (1992) Self-stimulation and drug reward mechanisms. Ann N Y Acad Sci 654:192-198.

Wolfman SL, Gill DF, Bogdanic F, Long K, Al-Hasani R, McCall JG, Bruchas MR, and McGehee DS (2018) Nicotine aversion is mediated by GABAergic interpeduncular nucleus inputs to laterodorsal tegmentum. Nat Commun 9:2710.

Wonnacott S (1997) Presynaptic nicotinic ACh receptors. Trends Neurosci 20:92-98

Xiao C, Cho JR, Zhou C, Treweek JB, Chan K, McKinney SL, Yang B, and Gradinaru V (2016) Cholinergic mesopontine signals govern locomotion and reward through dissociable midbrain pathways. Neuron 90:333-347.

Xie W, Kathuria H, Galiatsatos P, Blaha MJ, Hamburg NM, Robertson RM, Bhatnagar A, Benjamin EJ, and Stokes AC (2020) Association of electronic cigarette use with incident respiratory conditions among US adults from 2013 to 2018. JAMA Netw Open 3:e2020816.

Xu C, Sun Y, Cai X, You T, Zhao H, Li Y, and Zhao H (2018) Medial habenulainterpeduncular nucleus circuit contributes to anhedonia-like behavior in a rat model of depression. Front Behav Neurosci 12:238.

Yamaguchi T, Danjo T, Pastan I, Hikida T, and Nakanishi S (2013) Distinct roles of segregated transmission of the septo-habenular pathway in anxiety and fear. Neuron 78:537-544.

Yan Y, Beckley NA, Kim VJ, and Drenan RM (2019) Differential nicotinic modulation of glutamatergic and GABAergic VTA microcircuits. eNeuro 6:6.

Yan Y, Peng C, Arvin MC, Jin XT, Kim VJ, Ramsey MD, Wang Y, Banala S, Wokosin DL, McIntosh JM, et al. (2018) Nicotinic cholinergic receptors in VTA glutamate neurons modulate excitatory transmission. Cell Rep 23 $2236-2244$.

Yang K, Buhlman L, Khan GM, Nichols RA, Jin G, McIntosh JM, Whiteaker P, Lukas RJ, and Wu J (2011) Functional nicotinic acetylcholine receptors containing $\alpha 6$ subunits are on GABAergic neuronal boutons adherent to ventral tegmental area dopamine neurons. J Neurosci 31:2537-2548.

Yang K, Hu J, Lucero L, Liu Q, Zheng C, Zhen X, Jin G, Lukas RJ, and Wu J (2009) Distinctive nicotinic acetylcholine receptor functional phenotypes of rat ventral tegmental area dopaminergic neurons. J Physiol 587:345-361.

Yi F, Brubaker PL, and Jin T (2005) TCF-4 mediates cell type-specific regulation of proglucagon gene expression by beta-catenin and glycogen synthase kinase3beta. J Biol Chem 280:1457-1464.

Yildirim E, Connor DA, and Gould TJ (2015) ABT-089, but not ABT-107, ameliorates nicotine withdrawal-induced cognitive deficits in C57BL6/J mice. Behav Pharmacol 26:241-248.

Yoshimura RF, Hogenkamp DJ, Li WY, Tran MB, Belluzzi JD, Whittemore ER, Leslie FM, and Gee KW (2007) Negative allosteric modulation of nicotinic 
acetylcholine receptors blocks nicotine self-administration in rats. J Pharmacol Exp Ther 323:907-915.

Zangen A, Ikemoto S, Zadina JE, and Wise RA (2002) Rewarding and psychomotor stimulant effects of endomorphin-1: anteroposterior differences within the ventral tegmental area and lack of effect in nucleus accumbens. J Neurosci 22:7225-7233.

Zhang H and Sulzer D (2004) Frequency-dependent modulation of dopamine release by nicotine. Nat Neurosci 7:581-582.

Zhang J, Tan L, Ren Y, Liang J, Lin R, Feng Q, Zhou J, Hu F, Ren J, Wei C, et al. (2016) Presynaptic excitation via GABAB receptors in habenula cholinergic neurons regulates fear memory expression. Cell 166:716-728.

Zhao-Shea R, DeGroot SR, Liu L, Vallaster M, Pang X, Su Q, Gao G, Rando OJ, Martin GE, George O, et al. (2015) Increased CRF signalling in a ventral tegmental area-interpeduncular nucleus-medial habenula circuit induces anxiety during nicotine withdrawal. Nat Commun 6:6770

Zhao-Shea R, Liu L, Pang X, Gardner PD, and Tapper AR (2013) Activation of GABAergic neurons in the interpeduncular nucleus triggers physical nicotine withdrawal symptoms. Curr Biol 23:2327-2335.
Zhao-Shea R, Liu L, Soll LG, Improgo MR, Meyers EE, McIntosh JM, Grady SR, Marks MJ, Gardner PD, and Tapper AR (2011) Nicotine-mediated activation of dopaminergic neurons in distinct regions of the ventral tegmental area Neuropsychopharmacology 36:1021-1032.

Zhou FM, Liang Y, and Dani JA (2001) Endogenous nicotinic cholinergic activity regulates dopamine release in the striatum. Nat Neurosci 4:1224-1229.

Zoli M, Léna C, Picciotto MR, and Changeux JP (1998) Identification of four classes of brain nicotinic receptors using beta2 mutant mice. $J$ Neurosci 18:4461-4472.

Zoli M, Moretti M, Zanardi A, McIntosh JM, Clementi F, and Gotti C (2002) Identification of the nicotinic receptor subtypes expressed on dopaminergic terminals in the rat striatum. $J$ Neurosci 22:8785-8789.

Zuo W, Xiao C, Gao M, Hopf FW, Krnjević K, McIntosh JM, Fu R, Wu J, Bekker A and Ye JH (2016) Nicotine regulates activity of lateral habenula neurons via presynaptic and postsynaptic mechanisms. Sci Rep 6:32937.

Zwart R and Vijverberg HP (1998) Four pharmacologically distinct subtypes of alpha4beta2 nicotinic acetylcholine receptor expressed in Xenopus laevis oocytes. Mol Pharmacol 54:1124-1131. 